NATIONAL LABORATORY

\title{
Vehicle Technologies' Fact of the Week 2011
}

\section{April 2012}

Prepared by

Stacy C. Davis

Susan W. Diegel

Oak Ridge National Laboratory

Robert G. Boundy

Roltek, Inc.

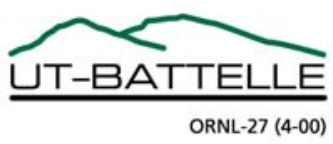




\section{DOCUMENT AVAILABILITY}

Reports produced after January 1, 1996, are generally available free via the U.S. Department of Energy (DOE) Information Bridge:

Web site: http://www.osti.gov/bridge

Reports produced before January 1, 1996, may be purchased by members of the public from the following source:

National Technical Information Service

5285 Port Royal Road

Springfield, VA 22161

Telephone: 703-605-6000 (1-800-553-6847)

TDD: 703-487-4639

Fax: 703-605-6900

E-mail: info@ntis.fedworld.gov

Web site: http://www.ntis.gov/support/ordernowabout.htm

Reports are available to DOE employees, DOE contractors, Energy Technology Data Exchange (ETDE) representatives, and International Nuclear Information System (INIS) representatives from the following source:

Office of Scientific and Technical Information

P.O. Box 62

Oak Ridge, TN 37831

Telephone: 865-576-8401

Fax: 865-576-5728

E-mail: reports@adonis.osti.gov

Web site: http://www.osti.gov/contact.html

This report was prepared as an account of work sponsored by an agency of the United States Government. Neither the United States government nor any agency thereof, nor any of their employees, makes any warranty, express or implied, or assumes any legal liability or responsibility for the accuracy, completeness, or usefulness of any information, apparatus, product, or process disclosed, or represents that its use would not infringe privately owned rights. Reference herein to any specific commercial product, process, or service by trade name, trademark, manufacturer, or otherwise, does not necessarily constitute or imply its endorsement, recommendation, or favoring by the United States Government or any agency thereof. The views and opinions of authors expressed herein do not necessarily state or reflect those of the United States Government or any agency thereof. 


\title{
FACT OF THE WEEK 2011
}

\author{
Stacy C. Davis \\ Susan W. Diegel \\ Oak Ridge National Laboratory \\ Robert G. Boundy \\ Roltek, Inc.
}

December 2011

\begin{abstract}
Prepared for the
Vehicle Technologies Program

Office of Energy Efficiency and Renewable Energy

U.S. Department of Energy
\end{abstract}

\author{
Prepared by the \\ Oak Ridge National Laboratory \\ Oak Ridge, Tennessee 37831-6073 \\ Managed by \\ UT-BATTELLE, LLC \\ for the \\ U.S. DEPARTMENT OF ENERGY \\ under Contract No. DE-AC05-00OR22725
}





\section{CONTENTS}

Page

ACKNOWLEDGEMENTS INTRODUCTION

... $\mathrm{v}$

vii

Fact Date

656 Jan 3 Consumers Hold onto Vehicles Longer .......................................... 1

657 Jan 10 Record Increase for New Light Vehicle Fuel Economy......................... 3

658 Jan 17 Increasing Use of Vehicle Technologies to Meet Fuel Economy

Requirements .................................................................. 6

659 Jan 24 Fuel Economy Ratings for Vehicles Operating on Electricity................ 8

660 Jan 31 Light Vehicle Sales Rise in 2010 .............................................. 10

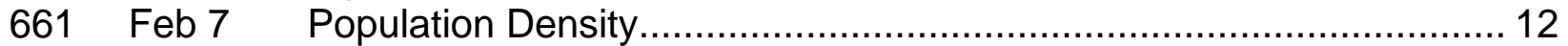

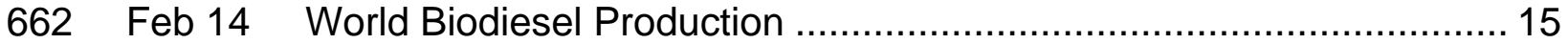

663 Feb 21 Clean Cities Program Petroleum Displacement Estimates for 2009... 17

664 Feb $28 \quad 2010$ U.S. Petroleum Imports by Country ........................................ 19

665 Mar $7 \quad$ Garage Availability for Plug-in Vehicles.......................................... 21

666 Mar 14 Survey says electric Vehicle Prices Are Key …................................ 23

667 Mar 21 Fuel Wasted in Traffic Congestion................................................. 26

668 Mar 28 Time Wasted Due to Traffic Congestion .......................................... 28

669 Apr $4 \quad$ GM Sells More Vehicles in China than in the U.S. ........................... 30

670 Apr 11 Vehicle-Miles of Travel Rises in 2010........................................... 32

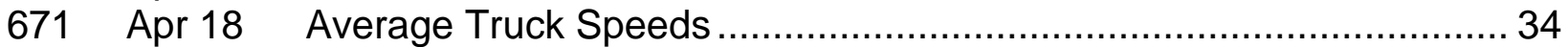

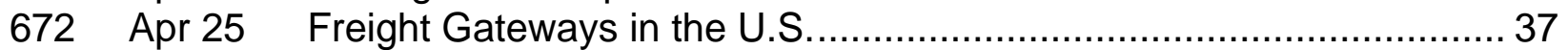

673 May $2 \quad$ U.S. Trade Balance for Transportation Vehicles ............................. 40

674 May 9 Petroleum Trade Balance .......................................................... 42

675 May 16 Gasoline Prices by Region, May 2, 2011...................................... 45

676 May 23 U.S. Refiners Produce about 19 Gallons of Gasoline from a

Barrel of Oil .......................................................................... 47

677 May 30 Number of Hybrid Models, 2001-2011......................................... 49

678 Jun $6 \quad$ Manufacturer Market Share of Hybrid Vehicles, 2010 ....................... 51

679 Jun $13 \quad$ U.S. Imports of Fuel Ethanol Drop Sharply..................................... 53

680 Jun 20 Fuel Economy is "Most Important" When Buying a Vehicle................ 55

681 Jun $27 \quad$ U.S. Ethanol Production, 2001-2010 …........................................ 57

682 Jul $4 \quad$ Federal Alternative Fuel Use ...................................................... 59

683 Jul 11 Federal Tax Credits for the Purchase of Advanced Technology

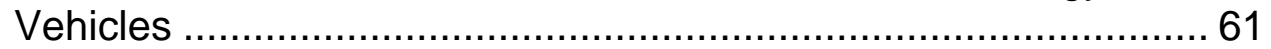

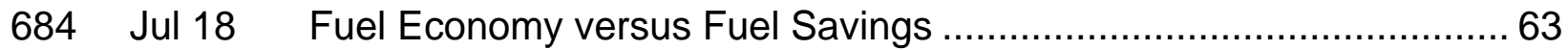

685 Jul 25 Reasons for Buying a Plug-in Hybrid Vehicle .....................................6 65

686 Aug 1 Emissions and Energy Use Model - GREET ..................................6 67

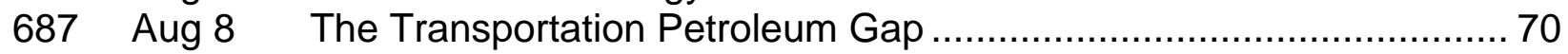

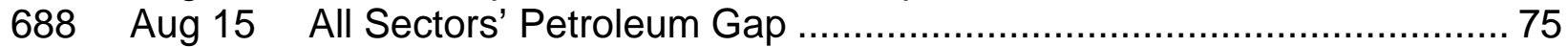

689 Aug 22 Energy Use by Sector and Source .............................................. 79

690 Aug 29 Characteristics of New Light Vehicles over Time .............................. 81 
691 Sep 5 Mexico Surpassed Canada in Vehicle Production ............................... 84

692 Sep 12 Fuel Economy Distribution for New Cars and Light Trucks ................ 86

693 Sep 19 Average Vehicle Footprint for Cars and Light Trucks ........................ 91

694 Sep 26 Costs of Owning a Vehicle by State .............................................. 93

695 Oct $3 \quad$ New Car Dealerships....................................................................... 97

696 Oct 10 Top Ten "Real World" Fuel Economy Leaders ................................. 99

697 Oct 17 Comparison of Vehicles per Thousand People in Selected

Countries/Regions............................................................... 101

698 Oct 24 Changes in the Federal Highway Administration Vehicle

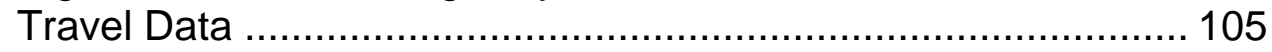

699 Oct 31 Transportation Energy Use by Mode and Fuel Type, 2009 .............. 108

$700 \quad$ Nov $7 \quad$ Biodiesel Consumption is on the Rise for $2011 \ldots \ldots \ldots \ldots \ldots \ldots \ldots \ldots \ldots \ldots . . . . . . . . . . . .110$

701 Nov 14 How Much More Would You Pay for an Electric Vehicle? ................. 112

702 Nov 21 Consumer Preferences on Electric Vehicle Charging ....................... 114

703 Nov 28 Hybrid Vehicles Loses Market Share in 2010 ............................... 116

704 Dec 5 Fuel Consumption Standards for New Heavy Duty Pickups

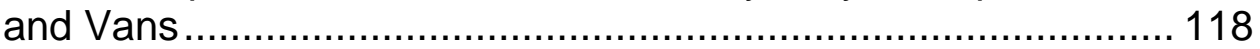

705 Dec 12 Fuel Consumption Standards for Combination Tractors ................... 121

706 Dec 19 Vocational Vehicle Fuel Consumption Standards........................... 123

707 Dec 26 Illustration of Truck Classes ......................................................... 125 


\section{ACKNOWLEDGEMENTS}

The authors would like to thank the project sponsors, Phil Patterson and Jake Ward, for their continued support and review of each weekly Fact. In addition, we thank Vicki Skonicki at Argonne National Laboratory for her invaluable role in posting the Facts on the Department of Energy's Vehicle Technologies Program website each week. 


\section{INTRODUCTION}

Each week the U.S. Department of Energy's Vehicle Technology Program (VTP) posts a Fact of the Week on their website: http://www1.eere.energy.gov/vehiclesandfuels/ . These Facts provide statistical information, usually in the form of charts and tables, on vehicle sales, fuel economy, gasoline prices, and other transportation-related trends. Each Fact is a stand-alone page that includes a graph, text explaining the significance of the data, the supporting information on which the graph was based, and the source of the data. A link to the current Fact is available Monday through Friday on the VTP homepage, but older Facts are archived and still available at:

http://www1.eere.energy.gov/vehiclesandfuels/facts/.

This report is a compilation of the Facts that were posted during calendar year 2011. The Facts were written and prepared by staff in Oak Ridge National Laboratory's Center for Transportation Analysis. 


\section{u.s. ofpartment of | Energy Efficiency \& \\ Renewable Energy}

\section{Vehicle Technologies Program}

\section{Fact \#656: January 3, 2011}

\section{Consumers Hold onto Vehicles Longer}

Consumers are holding onto both their new and used vehicles for longer periods of time. The length of time a consumer keeps a new vehicle has risen each quarter since 2008 to an average of 63.9 months in the 2 nd quarter of 2010 . That is 4.5 months longer than the same quarter last year. For used vehicles, the average length is 46.1 months, up 2.3 months from the 2nd quarter of 2009.

Average Length of Vehicle Ownership by Quarter, 2008 - 2010

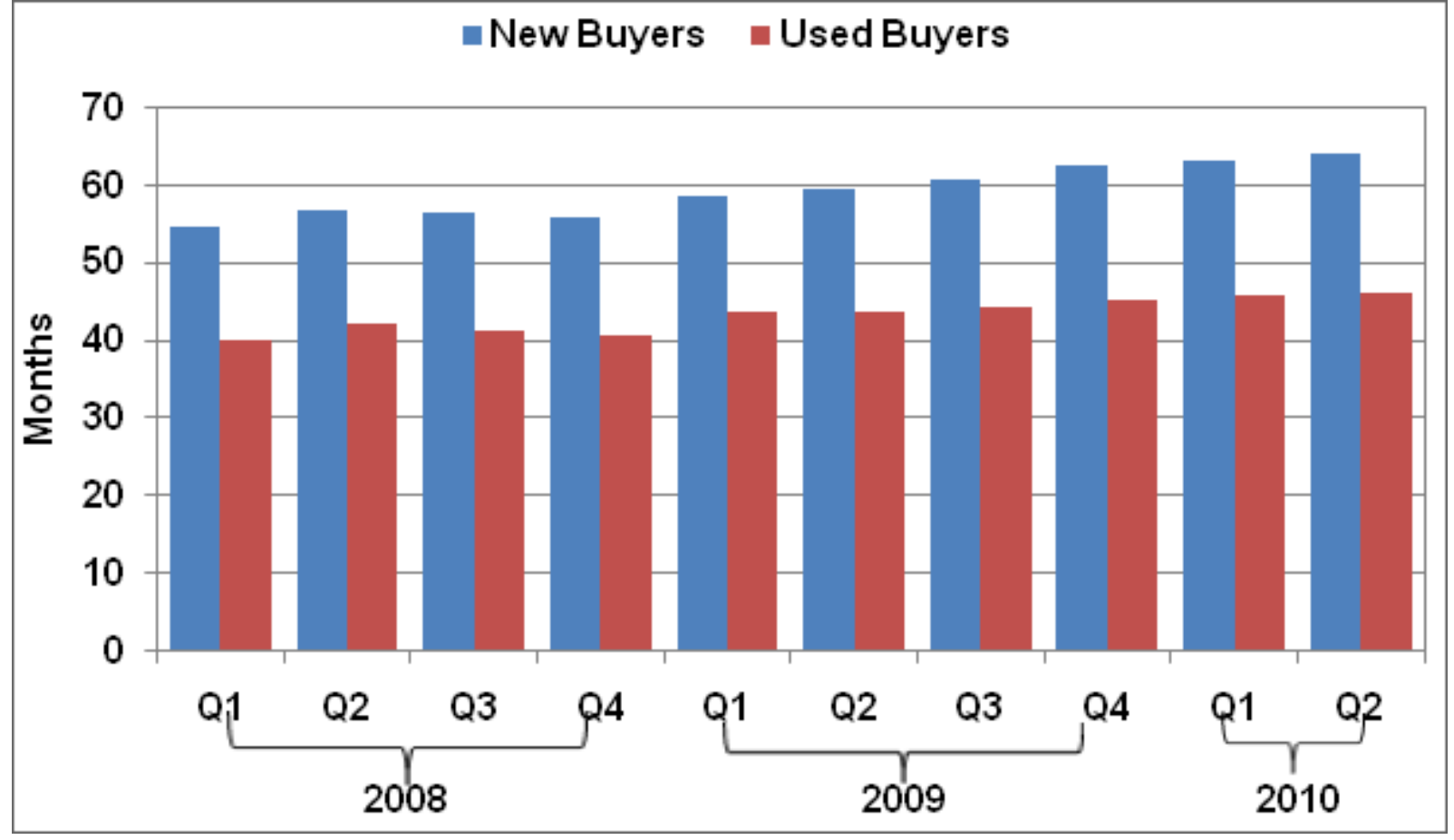




\section{Supporting Information}

\begin{tabular}{|c|c|c|c|}
\hline \multicolumn{4}{|c|}{$\begin{array}{l}\text { Average Length of Vehicle } \\
\text { Ownership } \\
\text { Quarter 1, } 2008 \text { - Quarter 2, } \\
2010\end{array}$} \\
\hline Year & Quarter & $\begin{array}{l}\text { New } \\
\text { Buyers } \\
\text { (Months) }\end{array}$ & $\begin{array}{l}\text { Used } \\
\text { Buyers } \\
\text { (Months) }\end{array}$ \\
\hline 2008 & Q1 & 54.6 & 40.0 \\
\hline 2008 & Q2 & 56.7 & 42.0 \\
\hline 2008 & Q3 & 56.3 & 41.3 \\
\hline 2008 & Q4 & 55.8 & 40.7 \\
\hline 2009 & Q1 & 58.6 & 43.7 \\
\hline 2009 & Q2 & 59.4 & 43.8 \\
\hline 2009 & Q3 & 60.6 & 44.2 \\
\hline 2009 & Q4 & 62.4 & 45.3 \\
\hline 2010 & Q1 & 63.2 & 45.9 \\
\hline 2010 & Q2 & 63.9 & 46.1 \\
\hline
\end{tabular}




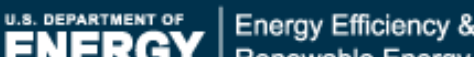 \\ Renewable Energy}

\section{Vehicle Technologies Program}

\section{Fact \#657: January 10, 2011 \\ Record Increase for New Light Vehicle Fuel Economy}

The sales-weighted fuel economy average of all light vehicles sold in model year (MY) 2009 was 1.4 miles per gallon (mpg) higher than MY2008. This is the largest annual increase in fuel economy since the Environmental Protection Agency (EPA) began recording new car fuel economy data in 1975. In addition, the $22.4 \mathrm{mpg}$ average in 2009 is the highest fuel economy in the series, topping the previous high of 22.0 in 1987. According to the EPA, fuel economy has moved through four phases over the years:

first, a rapid increase in fuel economy from 1975 to 1981 ;

second, a slower increase until reaching a peak in 1987;

third, a gradual decline until 2004; and

fourth, an increase each year since 2004, with the largest increase in 2009.

New Light Vehicle Fuel Economy, 1975 - 2009

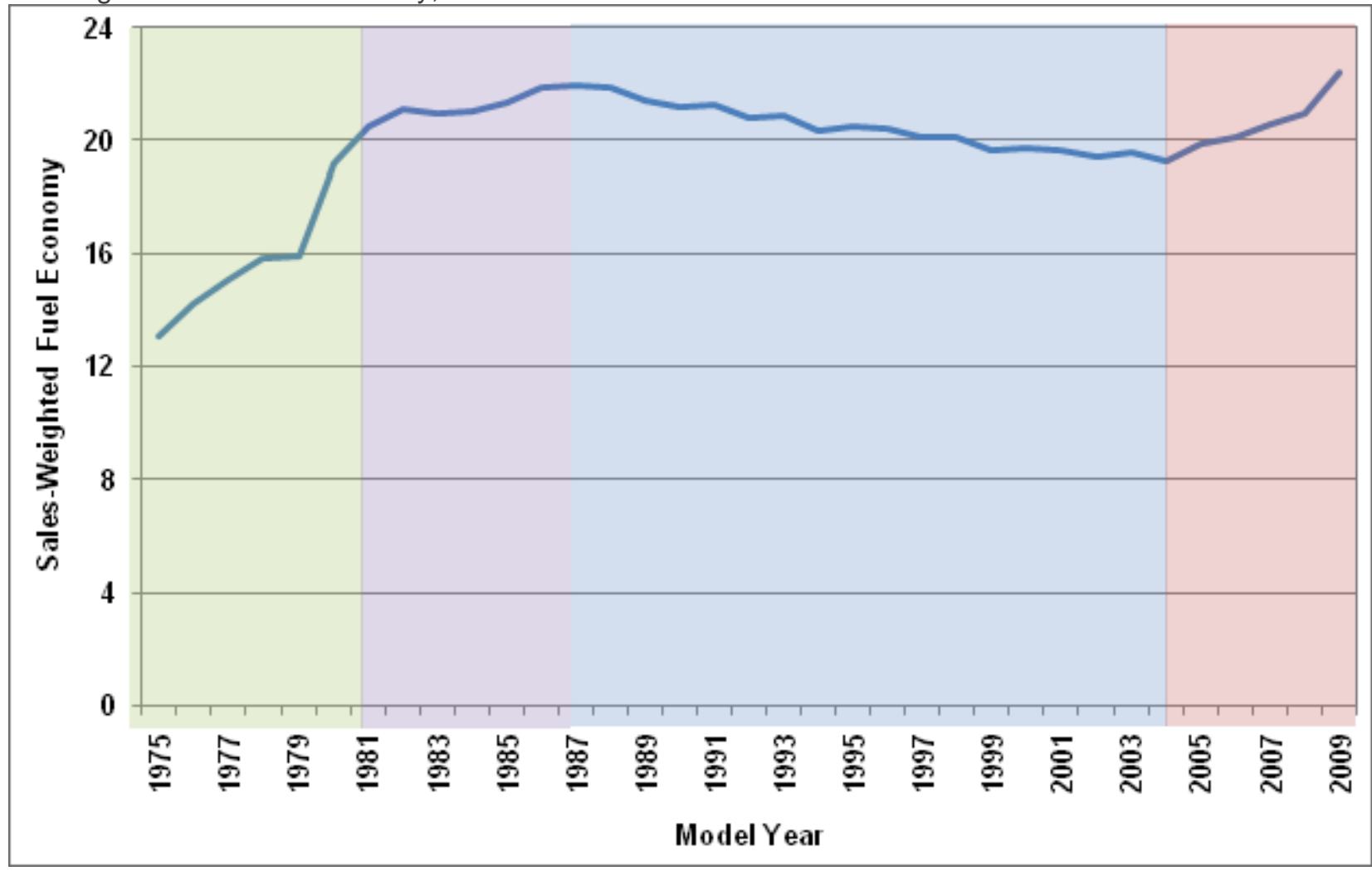

Note: Fuel economy data are EPA adjusted values. 


\section{Supporting Information}

\begin{tabular}{|l|l|}
\hline \multicolumn{2}{|c|}{ New Light Vehicle Fuel Economy, } \\
$1975-2009$
\end{tabular}




\begin{tabular}{|l|l|}
\hline \multicolumn{2}{|c|}{$\begin{array}{c}\text { New Light Vehicle Fuel Economy, } \\
1975-2009\end{array}$} \\
\hline Model Year & Miles per Gallon \\
\hline 2003 & 19.6 \\
\hline 2004 & 19.3 \\
\hline 2005 & 19.9 \\
\hline 2006 & 20.1 \\
\hline 2007 & 20.6 \\
\hline 2008 & 21.0 \\
\hline 2009 & 22.4 \\
\hline $\begin{array}{l}\text { Note: Fuel economy data are sales- } \\
\text { weighted EPA adjusted values. }\end{array}$ \\
$\begin{array}{l}\text { Source: EPA, Light-Duty Automotive } \\
\text { Technology, Carbon Dioxide Emissions, }\end{array}$ \\
\hline $\begin{array}{l}\text { and Fuel Economy Trends: 1975 } \\
\text { Through 2010, November 2010. }\end{array}$ \\
\hline
\end{tabular}




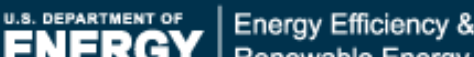

Renewable Energy

\section{Vehicle Technologies Program}

\section{Fact \#658: January 17, 2011 Increasing Use of Vehicle Technologies to Meet Fuel Economy Requirements}

Vehicle manufacturers are turning to vehicle technologies to improve efficiency and meet strict fuel economy requirements. Over the last 10 years, the use of engine technologies like multi-valves and variable valve timing have become commonplace while other technologies like gasoline direct injection, cylinder deactivation, hybrid drivetrains and continuously variable transmissions have gained market share, particularly in the last 5 years. Though not a new technology, turbochargers have improved in recent years and manufacturers are increasingly viewing them as a way to meet performance requirements with smaller engines. Ford's popular EcoBoost engines use a combination of turbocharging and gasoline direct injection and there are other manufacturers employing this strategy as well.

Market Share of Selected Vehicle Technologies for Improved Efficiency, 2001 - 2010

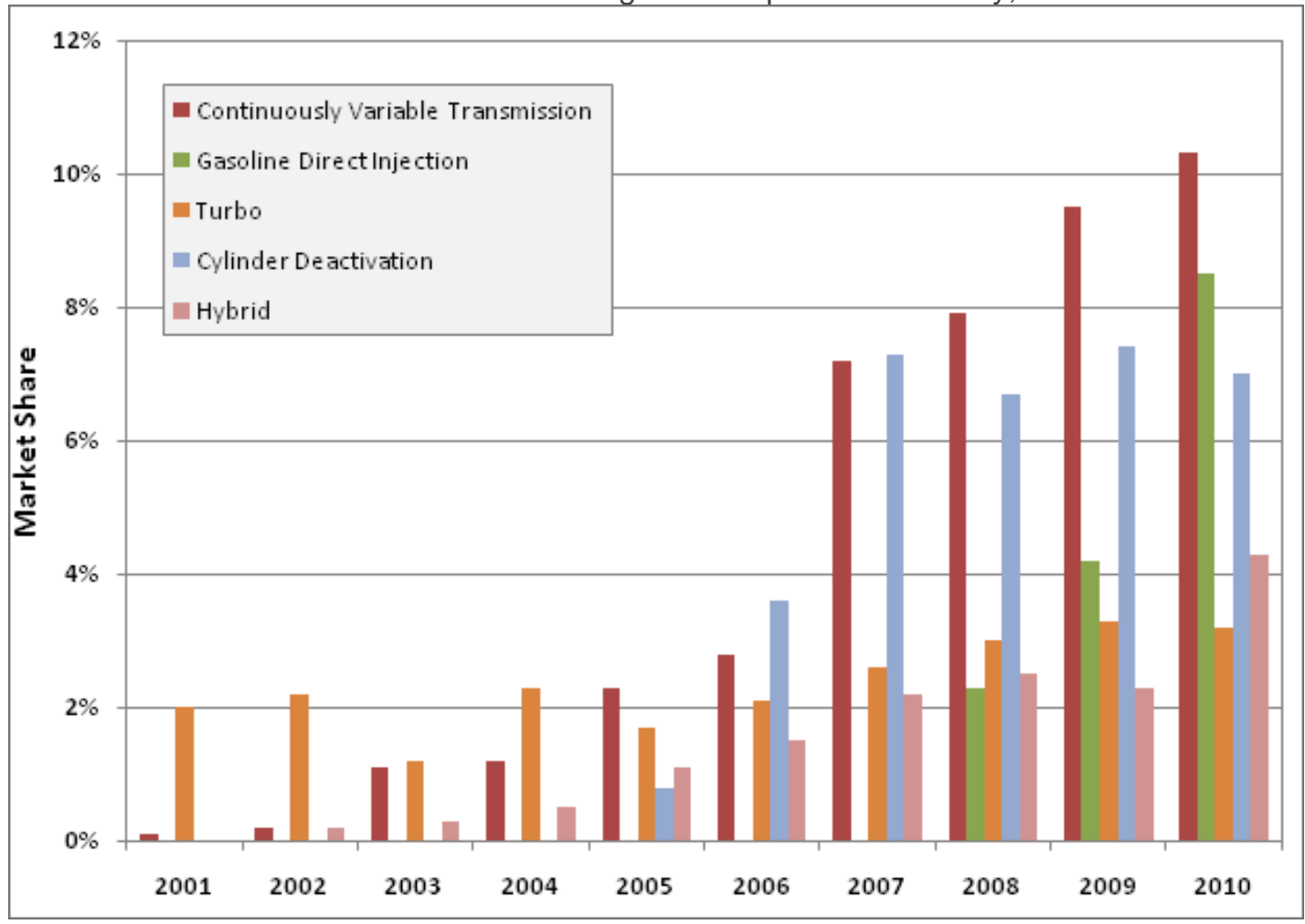

Source: Environmental Protection Agency, Light-Duty Automotive Technology, Carbon Dioxide Emissions, and Fuel Economy Trends: 1975 Through 2010. 


\section{Supporting Information}

\begin{tabular}{|c|c|c|c|c|c|c|c|c|c|c|}
\hline \multicolumn{11}{|c|}{ Leases as a Share of Total U.S. New Vehicle Sales, 2005-2010 } \\
\hline Technology & 2001 & 2002 & 2003 & 2004 & 2005 & 2006 & 2007 & 2008 & 2009 & 2010 \\
\hline $\begin{array}{l}\text { Continuously } \\
\text { Variable } \\
\text { Transmission }\end{array}$ & $0.1 \%$ & $0.2 \%$ & $1.1 \%$ & $1.2 \%$ & $2.3 \%$ & $2.8 \%$ & $7.2 \%$ & $7.9 \%$ & $9.5 \%$ & $10.3 \%$ \\
\hline $\begin{array}{l}\text { Gasoline } \\
\text { Direct } \\
\text { Injection }\end{array}$ & $0.0 \%$ & $0.0 \%$ & $0.0 \%$ & $0.0 \%$ & $0.0 \%$ & $0.0 \%$ & $0.0 \%$ & $2.3 \%$ & $4.2 \%$ & $8.5 \%$ \\
\hline Turbo & $2.0 \%$ & $2.2 \%$ & $1.2 \%$ & $2.3 \%$ & $1.7 \%$ & $2.1 \%$ & $2.6 \%$ & $3.0 \%$ & $3.3 \%$ & $3.2 \%$ \\
\hline $\begin{array}{l}\text { Cylinder } \\
\text { Deactivation }\end{array}$ & $0.0 \%$ & $0.0 \%$ & $0.0 \%$ & $0.0 \%$ & $0.8 \%$ & $3.6 \%$ & $7.3 \%$ & $6.7 \%$ & $7.4 \%$ & $7.0 \%$ \\
\hline Hybrid & $0.0 \%$ & $0.2 \%$ & $0.3 \%$ & $0.5 \%$ & $1.1 \%$ & $1.5 \%$ & $2.2 \%$ & $2.5 \%$ & $2.3 \%$ & $4.3 \%$ \\
\hline Multi-Valve* & $49.0 \%$ & $53.3 \%$ & $55.5 \%$ & $62.3 \%$ & $65.6 \%$ & $71.7 \%$ & $71.7 \%$ & $76.4 \%$ & $83.6 \%$ & $86.1 \%$ \\
\hline $\begin{array}{l}\text { Variable } \\
\text { Valve } \\
\text { Timing* }\end{array}$ & $19.6 \%$ & $25.3 \%$ & $30.6 \%$ & $38.5 \%$ & $45.8 \%$ & $55.4 \%$ & $57.3 \%$ & $58.2 \%$ & $72.0 \%$ & $86.4 \%$ \\
\hline
\end{tabular}




\section{Vehicle Technologies Program}

Fact \#659: January 24, 2011

\section{Fuel Economy Ratings for Vehicles Operating on Electricity}

The Environmental Protection Agency has developed a new methodology for determining how fuel economy information will be displayed on the window sticker of a vehicle that operates on electricity. The fuel economy will be displayed in miles per gallon equivalent (MPGequivalent), so that consumers can compare the pure electric operating efficiency against traditional vehicles in the same class. The vehicle's range on electricity will also be displayed on the sticker. The calculation of MPGequivalent is based on 33.7 kilowatt-hours (kW-hrs) of electricity being equivalent to one gallon of gasoline, or 33.7kW-hrs = 1 MPGequivalent. The all-electric Nissan Leaf and the plug-in hybrid-electric Chevrolet Volt are the first two vehicles to use the new window sticker format. The Volt also has a fuel economy for driving in gasoline-only mode, which is measured in the familiar miles per gallon (MPG).

Example Window Stickers for Vehicles Operating on Electricity

Nissan Leaf

\section{EPA Fuel Economy and} Environmental Comparisons

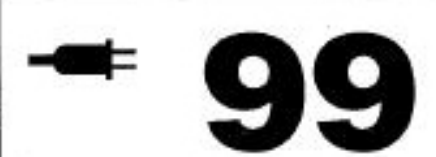

combined city/hwy

\section{MPG equivalent

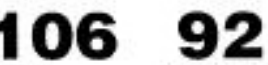 \\ city \\ highway}

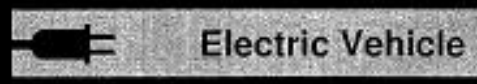

Annual Electric Cost

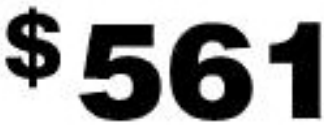

Charge \& Range

Ful Battory Charge Time

(1) 7 hours on a fuly changed bettery, vehicle can travel about. $\begin{array}{llllllll}\text { at } 240 \mathrm{~V} & 0 & 7 & 14 & 21 & 28 & 35 & 42\end{array}$
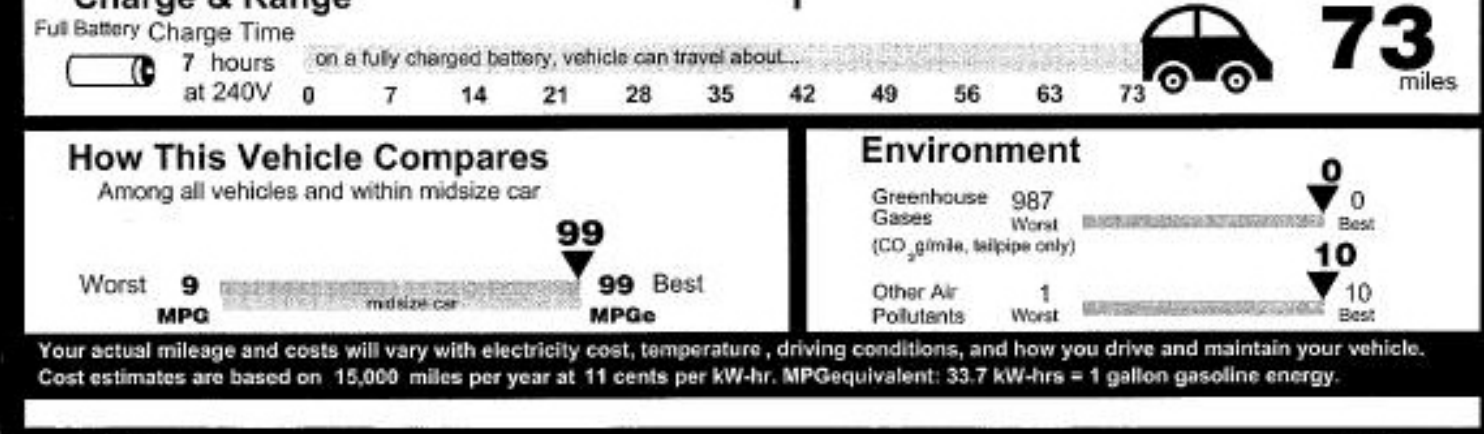


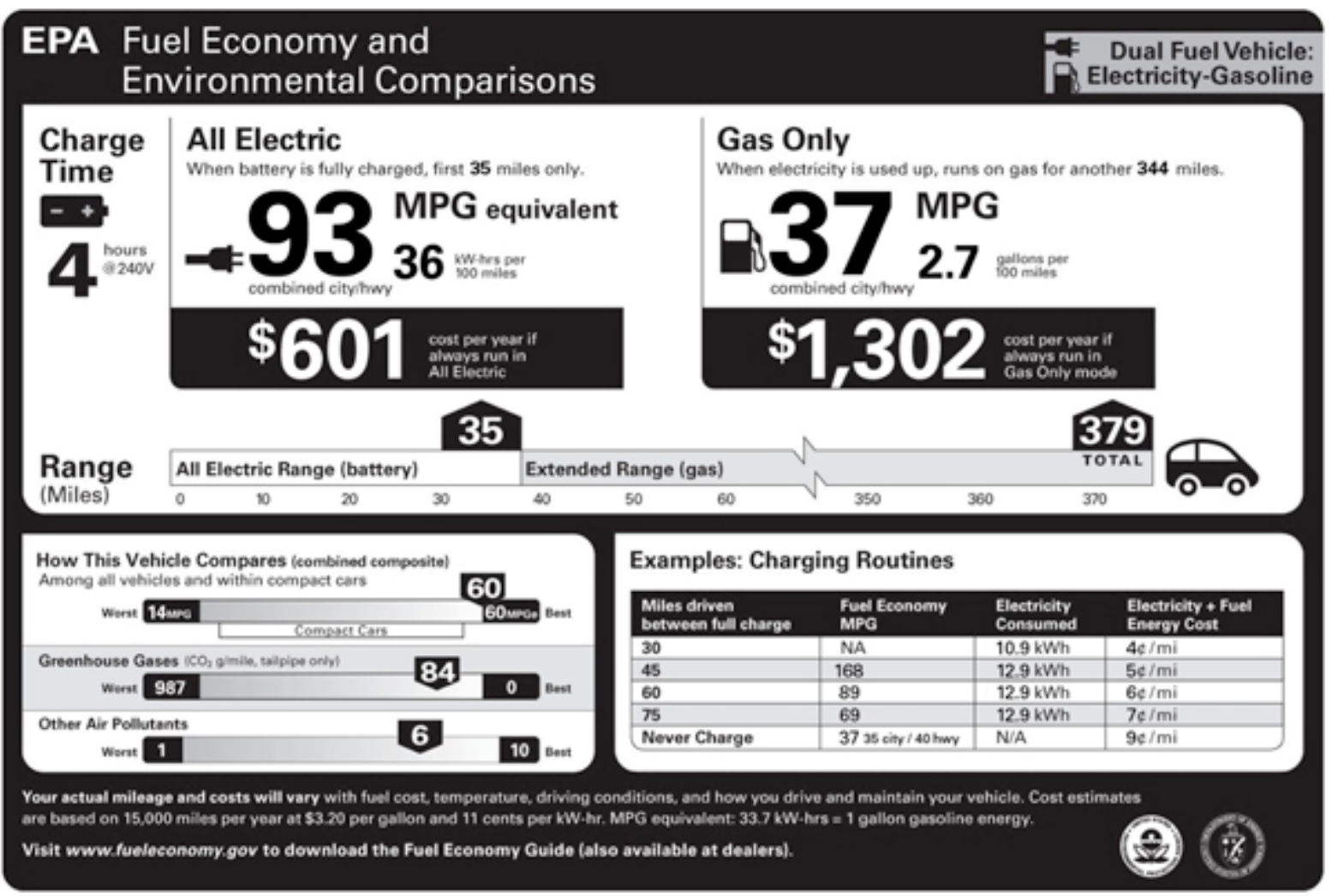

\section{Supporting Information}

The Nissan Leaf EPA Window Sticker shows 99 MPGequivalent (combined city/highway) and a range of 73 miles. The Chevy Volt EPA Window Sticker shows 93 MPGequivalent (combined city/highway) in all electric mode and 37 MPG in gas only mode. The range of the Chevy Volt is 35 miles on electricity and 379 miles total (combining gasoline and electric range).

Source: U.S. Environmental Protection Agency 


\section{u.s. oppaprusury of | Energy Efficiency \& \\ Renewable Energy}

\section{Vehicle Technologies Program}

Fact \#660: January 31, 2011

Light Vehicle Sales Rise in 2010

The total sales of light vehicles (cars and light trucks) in the U.S. have ranged between 10 million and 17 million over the course of the last 40 years. Though the sales have experienced highs and lows over this period, the recent sales plummet from 16.1 million vehicles in 2007 to 10.4 million vehicles in 2009 was the largest drop in the 40 year period with sales reduced to the 1982 level. Light vehicle sales increased to 11.6 million in 2010 (preliminary estimate).

Light Vehicle Sales, 1970 - 2010

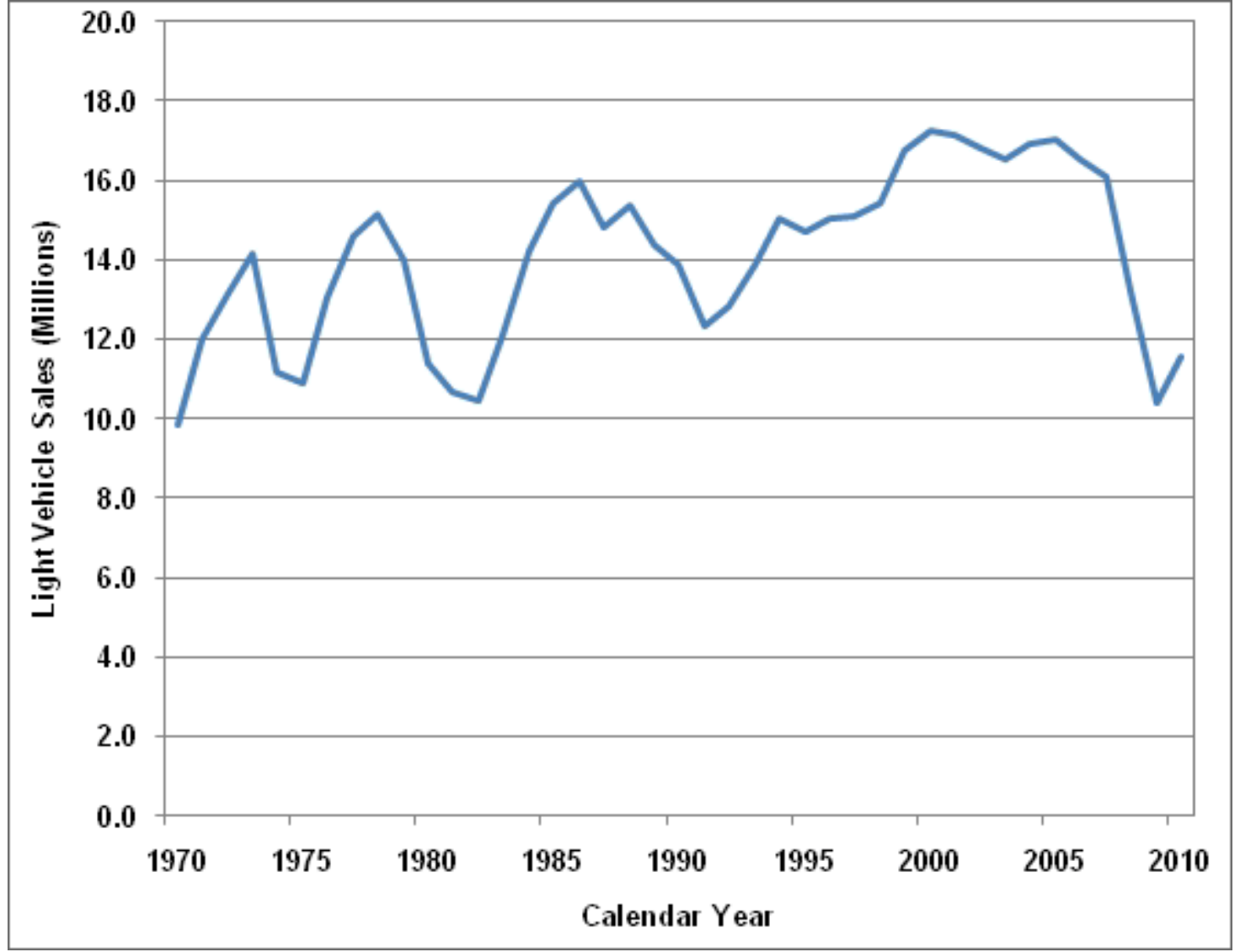




\section{Supporting Information}

\begin{tabular}{|c|c|c|c|}
\hline \multicolumn{4}{|c|}{ Light Vehicle Sales, 1970-2010 } \\
\hline $\begin{array}{l}\text { Calendar } \\
\text { Year }\end{array}$ & $\begin{array}{l}\text { Sales } \\
\text { (Millions) }\end{array}$ & $\begin{array}{l}\text { Calendar } \\
\text { Year }\end{array}$ & $\begin{array}{l}\text { Sales } \\
\text { (Millions) }\end{array}$ \\
\hline 1970 & 9.9 & 1990 & 13.9 \\
\hline 1971 & 12.0 & 1991 & 12.3 \\
\hline 1972 & 13.2 & 1992 & 12.8 \\
\hline 1973 & 14.2 & 1993 & 13.9 \\
\hline 1974 & 11.2 & 1994 & 15.0 \\
\hline 1975 & 10.9 & 1995 & 14.7 \\
\hline 1976 & 13.1 & 1996 & 15.0 \\
\hline 1977 & 14.6 & 1997 & 15.1 \\
\hline 1978 & 15.1 & 1998 & 15.4 \\
\hline 1979 & 14.0 & 1999 & 16.8 \\
\hline 1980 & 11.4 & 2000 & 17.2 \\
\hline 1981 & 10.7 & 2001 & 17.1 \\
\hline 1982 & 10.4 & 2002 & 16.8 \\
\hline 1983 & 12.1 & 2003 & 16.5 \\
\hline 1984 & 14.2 & 2004 & 16.9 \\
\hline 1985 & 15.4 & 2005 & 17.0 \\
\hline 1986 & 16.0 & 2006 & 16.5 \\
\hline 1987 & 14.8 & 2007 & 16.1 \\
\hline 1988 & 15.3 & 2008 & 13.2 \\
\hline \multirow[t]{2}{*}{1989} & 14.4 & 2009 & 10.4 \\
\hline & & 2010 & 11.6 \\
\hline $\begin{array}{l}\text { Note: } 2010 \\
\text { Source: W }\end{array}$ & \multicolumn{3}{|c|}{ Note: 2010 data are preliminary. } \\
\hline
\end{tabular}




\section{u.s. оеравтымent of | Energy Efficiency \&}

Renewable Energy

\section{Vehicle Technologies Program}

\section{Fact \#661: February 7, 2011 Population Density}

The density of the population in the U.S., measured as the number of people per square mile, affects the way goods and people are transported. The newly released 2010 Census data show that, on a state by state basis, the District of Columbia has the most dense population, followed by New Jersey and Rhode Island. In the western states, the population is generally less dense, with the exceptions being California, Hawaii, and Washington. Every state except Michigan experienced increased population density from the 2000 Census to the 2010 Census.

U.S. Population Density by State, 2010 (people per square mile)

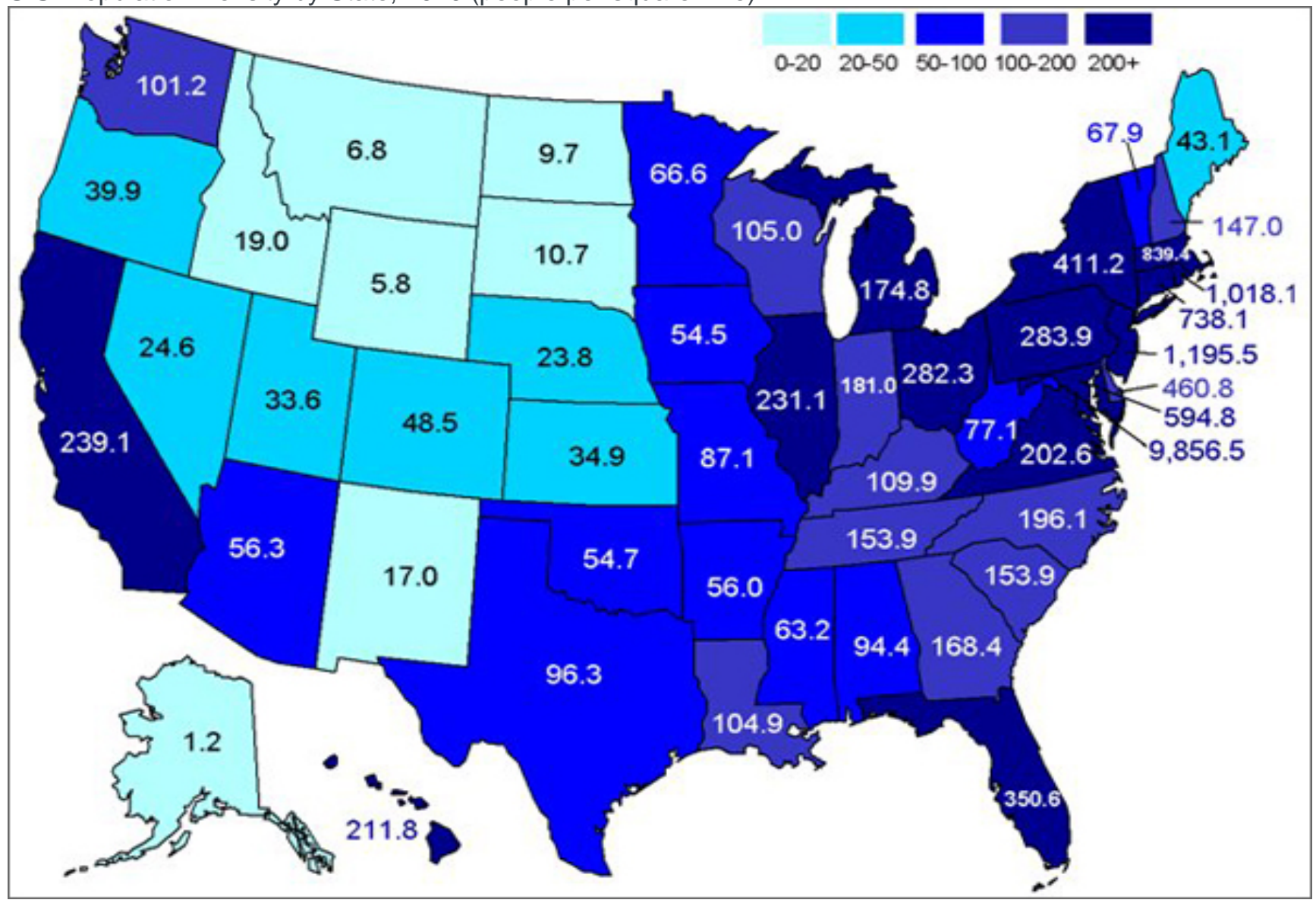

Note: Puerto Rico, a U.S. territory, was excluded from this analysis. 


\section{Supporting Information}

\begin{tabular}{|c|c|c|c|}
\hline \multicolumn{4}{|c|}{ U.S. Population, Population Density, and Rank by State, 2010} \\
\hline State & Population & $\begin{array}{l}\text { Population Density } \\
\text { (People per } \\
\text { Square Mile) }\end{array}$ & $\begin{array}{l}\text { Population Density } \\
\text { Rank }\end{array}$ \\
\hline $\begin{array}{l}\text { District of } \\
\text { Columbia }\end{array}$ & 601,723 & $9,856.5$ & 1 \\
\hline New Jersey & $8,791,894$ & $1,195.5$ & 2 \\
\hline Rhode Island & $1,052,567$ & $1,018.1$ & 3 \\
\hline Massachusetts & $6,547,629$ & 839.4 & 4 \\
\hline Connecticut & $3,574,097$ & 738.1 & 5 \\
\hline Maryland & $5,773,552$ & 594.8 & 6 \\
\hline Delaware & 897,934 & 460.8 & 7 \\
\hline New York & $19,378,102$ & 411.2 & 8 \\
\hline Florida & $18,801,310$ & 350.6 & 9 \\
\hline Pennsylvania & $12,702,379$ & 283.9 & 10 \\
\hline Ohio & $11,536,504$ & 282.3 & 11 \\
\hline California & $37,253,956$ & 239.1 & 12 \\
\hline Illinois & $12,830,632$ & 231.1 & 13 \\
\hline Hawaii & $1,360,301$ & 211.8 & 14 \\
\hline Virginia & $8,001,024$ & 202.6 & 15 \\
\hline North Carolina & $9,535,483$ & 196.1 & 16 \\
\hline Indiana & $6,483,802$ & 181.0 & 17 \\
\hline Michigan & $9,883,640$ & 174.8 & 18 \\
\hline Georgia & $9,687,653$ & 168.4 & 19 \\
\hline Tennessee & $6,346,105$ & 153.9 & 20 \\
\hline South Carolina & $4,625,364$ & 153.9 & 21 \\
\hline New Hampshire & $1,316,470$ & 147.0 & 22 \\
\hline Kentucky & $4,339,367$ & 109.9 & 23 \\
\hline Wisconsin & $5,686,986$ & 105.0 & 24 \\
\hline Louisiana & $4,533,372$ & 104.9 & 25 \\
\hline Washington & $6,724,540$ & 101.2 & 26 \\
\hline
\end{tabular}




\begin{tabular}{|c|c|c|c|}
\hline \multicolumn{4}{|c|}{ U.S. Population, Population Density, and Rank by State, 2010} \\
\hline State & Population & $\begin{array}{l}\text { Population } \\
\text { Density } \\
\text { (People per } \\
\text { Square Mile) }\end{array}$ & $\begin{array}{l}\text { Population Density } \\
\text { Rank }\end{array}$ \\
\hline Texas & $25,145,561$ & 96.3 & 27 \\
\hline Alabama & $4,779,736$ & 94.4 & 28 \\
\hline Missouri & $5,988,927$ & 87.1 & 29 \\
\hline West Virginia & $1,852,994$ & 77.1 & 30 \\
\hline Vermont & 625,741 & 67.9 & 31 \\
\hline Minnesota & $5,303,925$ & 66.6 & 32 \\
\hline Mississippi & $2,967,297$ & 63.2 & 33 \\
\hline Arizona & $6,392,017$ & 56.3 & 34 \\
\hline Arkansas & $2,915,918$ & 56.0 & 35 \\
\hline Oklahoma & $3,751,351$ & 54.7 & 36 \\
\hline lowa & $3,046,355$ & 54.5 & 37 \\
\hline Colorado & $5,029,196$ & 48.5 & 38 \\
\hline Maine & $1,328,361$ & 43.1 & 39 \\
\hline Oregon & $3,831,074$ & 39.9 & 40 \\
\hline Kansas & $2,853,118$ & 34.9 & 41 \\
\hline Utah & $2,763,885$ & 33.6 & 42 \\
\hline Nevada & $2,700,551$ & 24.6 & 43 \\
\hline Nebraska & $1,826,341$ & 23.8 & 44 \\
\hline Idaho & $1,567,582$ & 19.0 & 45 \\
\hline New Mexico & $2,059,179$ & 17.0 & 46 \\
\hline South Dakota & 814,180 & 10.7 & 47 \\
\hline North Dakota & 672,591 & 9.7 & 48 \\
\hline Montana & 989,415 & 6.8 & 49 \\
\hline Wyoming & 563,626 & 5.8 & 50 \\
\hline Alaska & 710,231 & 1.2 & 51 \\
\hline United States & $308,745,538$ & 87.4 & \\
\hline \multicolumn{4}{|c|}{ Note: Puerto Rico, a U.S. territory, was excluded from this analysis. } \\
\hline
\end{tabular}




\section{Vehicle Technologies Program}

\section{Fact \#662: February 14, 2011 World Biodiesel Production}

Europe has been the dominant region for biodiesel production with increased production each year since 2005. North America has been a distant second led by the United States until 2009. In 2009, U.S. biodiesel production fell by over 10 thousand barrels per day while continued growth in Central \& South America and Asia \& Oceania surpassed North America in production of biodiesel for the first time. The declining biodiesel production in the United States beginning in 2008 is likely due to changes in Federal subsidies for biodiesel as well as changes in foreign trade policy and the downturn of the economy.

World Biodiesel Production by Region and Selected Countries, 2005-2009 (Thousand barrels per day)

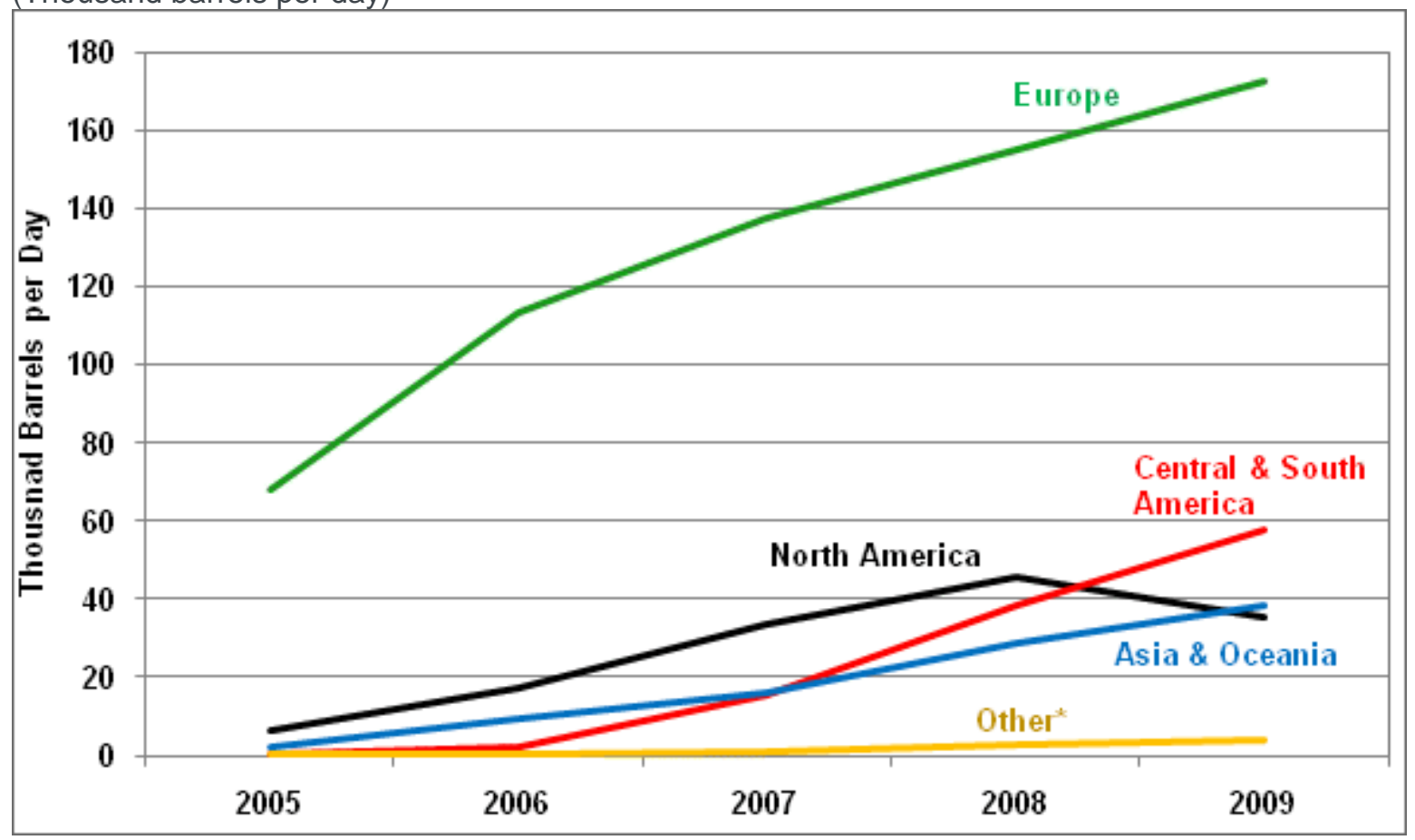




\section{Supporting Information}

\begin{tabular}{|c|c|c|c|c|c|}
\hline \multicolumn{6}{|c|}{$\begin{array}{c}\text { World Biodiesel Production by Region and Selected } \\
\text { Countries, 2005-2009 } \\
\text { (Thousand barrels per day) }\end{array}$} \\
\hline Region/Country & 2005 & 2006 & 2007 & 2008 & 2009 \\
\hline North America & 6.1 & 17.1 & 33.7 & 45.9 & 35.2 \\
\hline United States & 5.9 & 16.3 & 32.0 & 44.1 & 32.9 \\
\hline $\begin{array}{l}\text { Central \& South } \\
\text { America }\end{array}$ & 0.5 & 2.2 & 15.2 & 38.6 & 57.9 \\
\hline Europe & 68.1 & 113.2 & 137.5 & 155.0 & 172.6 \\
\hline Asia \& Oceania & 2.2 & 9.1 & 15.8 & 28.8 & 38.5 \\
\hline Other* & 0.3 & 0.3 & 0.7 & 2.5 & 3.9 \\
\hline World & 77.2 & 142.0 & 202.9 & 270.9 & 308.2 \\
\hline \multicolumn{6}{|c|}{$\begin{array}{l}\text { *Other includes Africa, Eurasia and the Middle East. } \\
\text { Source: Energy Information Administration, International Energy } \\
\text { Statistics, Biofuels Production. The above table was derived from an } \\
\text { interactive table generated on December } 9,2010 .\end{array}$} \\
\hline
\end{tabular}




\section{Vehicle Technologies Program}

\section{Fact \#663: February 21, 2011 Clean Cities Program Petroleum Displacement Estimates for 2009}

Each year, estimates are made of the amount of petroleum that is displaced by the efforts of the U.S. Department of Energy's Clean Cities Program. These estimates are based on data provided by Clean Cities Coalition coordinators around the country and serve as an indicator of the impact of the coalitions. According to the program estimates, advancing the role of alternative fuel vehicles had the largest effect on petroleum - 220.1 gasoline-gallon equivalents (GGE), or $69.8 \%$ of total displacement. Using biodiesel blends instead of $100 \%$ petroleum-based diesel accounted for 53.5 GGEs, which was $17 \%$ of petroleum displacement. Other petroleum-saving approaches included using hybrid electric vehicles in the place of traditional vehicles, using idle reduction technologies and programs, vehicle miles of travel (VMT) reduction programs, fuel economy projects, and using alternative fuels in off-road equipment.

Clean Cities Petroleum Displacement Estimates, 2009

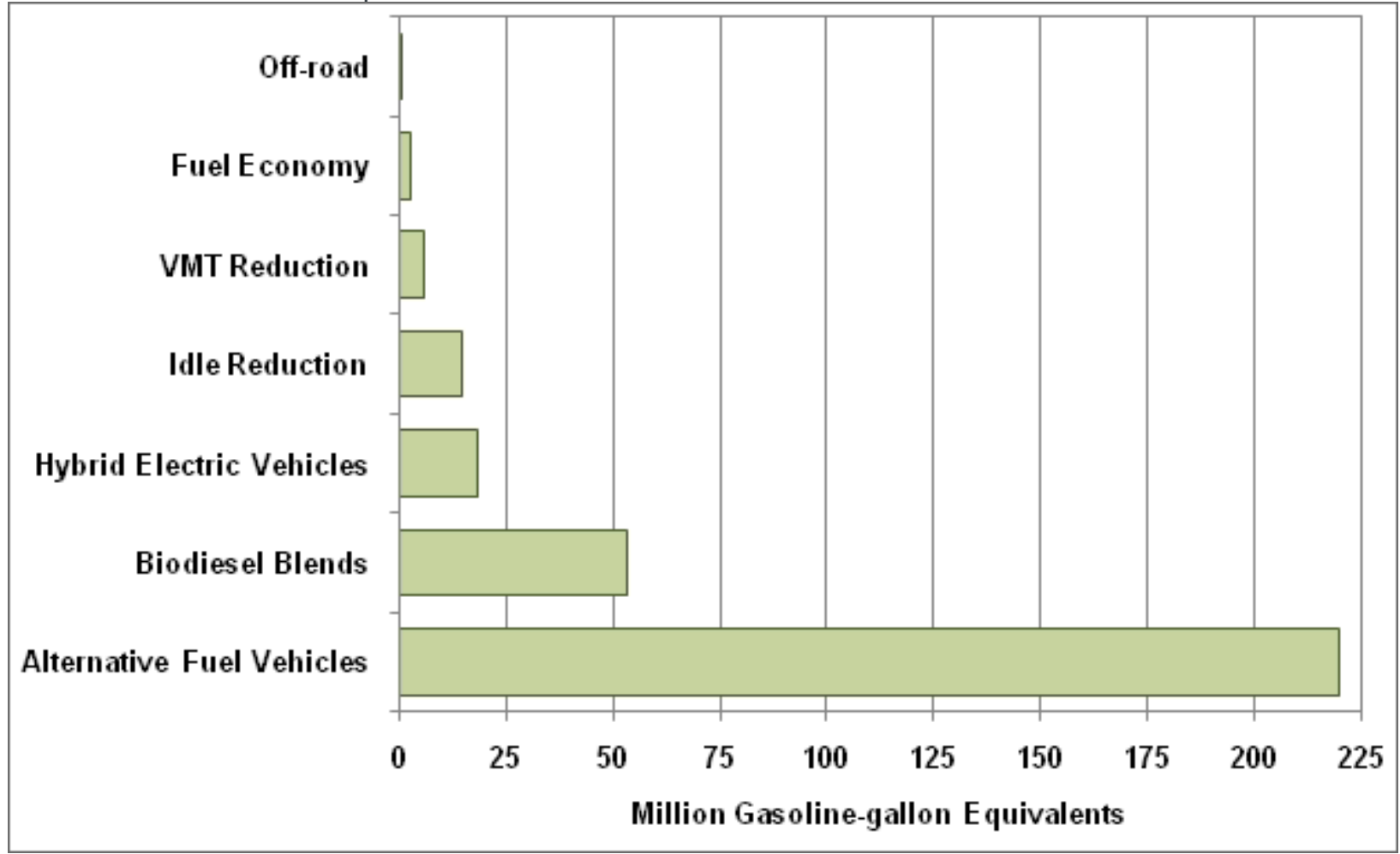




\section{Supporting Information}

\begin{tabular}{|l|c|c|}
\hline \multicolumn{1}{|c|}{ Clean Cities Petroleum Displacement Estimates by } \\
Technology
\end{tabular}




\section{U.s. ofpartment of | Energy Efficiency \& \\ Renewable Energy}

\section{Vehicle Technologies Program}

\section{Fact \#664: February 28, 2011}

\section{U.S. Petroleum Imports by Country}

The U.S. imported almost 12 million barrels per day in 2010, according to data for the first ten months of the year. Canada, Mexico and other non-OPEC countries are the top three places from which the U.S. imported petroleum. Saudi Arabia, Nigeria, and Venezuela - which are all OPEC nations - each provided the U.S. with about one million barrels per day of petroleum. Libya, also part of OPEC, provided the U.S. with only 76 thousand barrels per day.

U.S. Imports of Petroleum by Country, First Ten Months of 2010

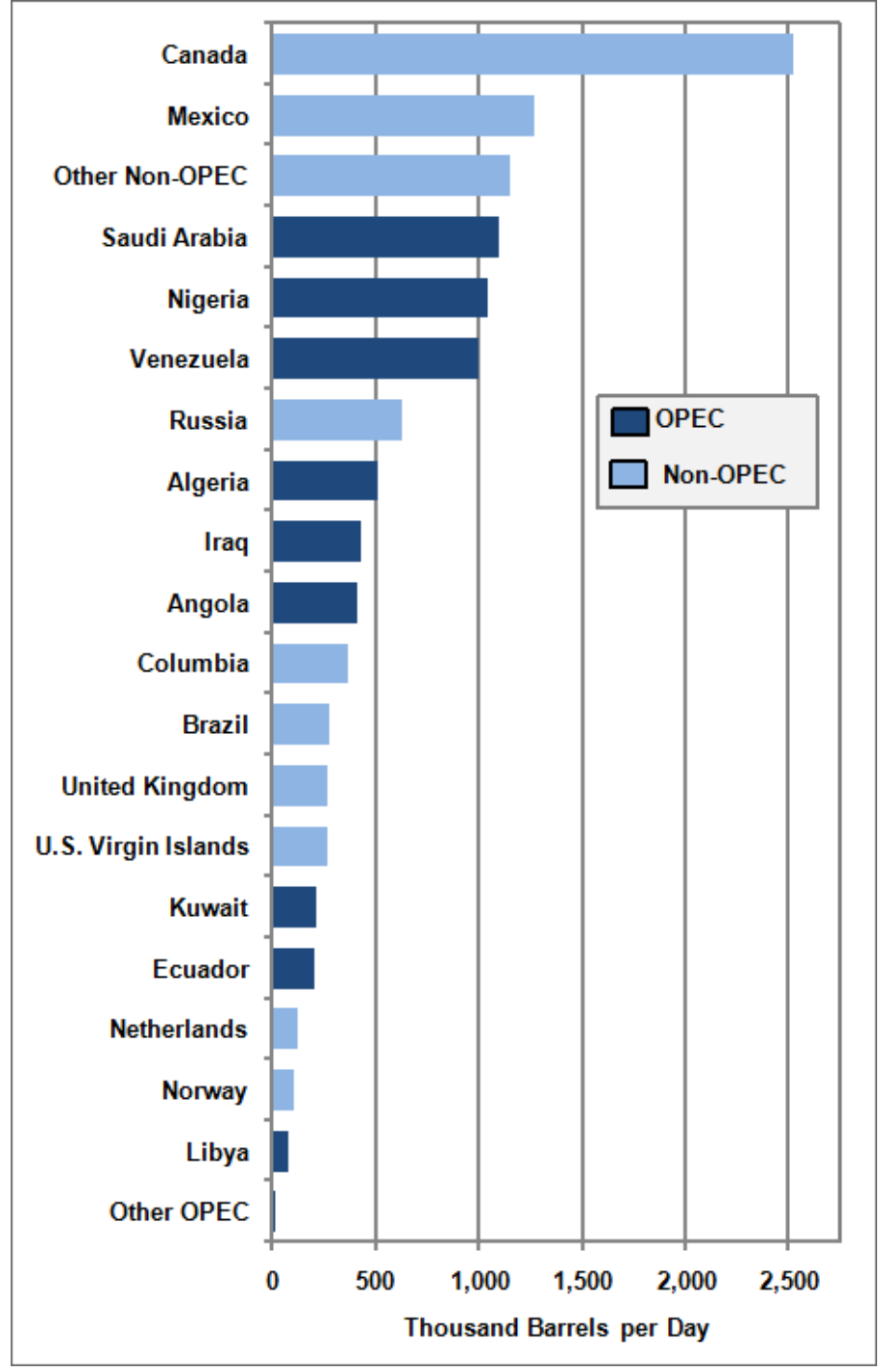




\section{Supporting Information}

\begin{tabular}{|l|r|l|r|}
\hline \multicolumn{3}{|c|}{ U.S. Petroleum Imports, First Ten Months of 2010 } \\
(Thousand Barrels per Day)
\end{tabular}




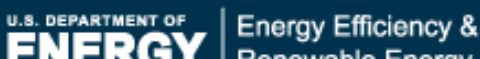 \\ Renewable Energy}

\section{Vehicle Technologies Program}

\section{Fact \#665: March 7, 2011 Garage Availability for Plug-in Vehicles}

According to the 2009 American Housing Survey, two-thirds of all housing units in the U.S. have a garage or carport. The access to electricity that a garage or carport may provide is important for the light vehicle manufacturers who are selling or planning to sell electric vehicles or plug-in hybrid vehicles. The good news for those manufacturers is that $82.3 \%$ of newly constructed housing units (4 years old or less) have a garage or carport. Those living in manufactured housing/mobile homes are the least likely to have a garage or carport.

Share of Housing Units with a Garage or Carport, 2009

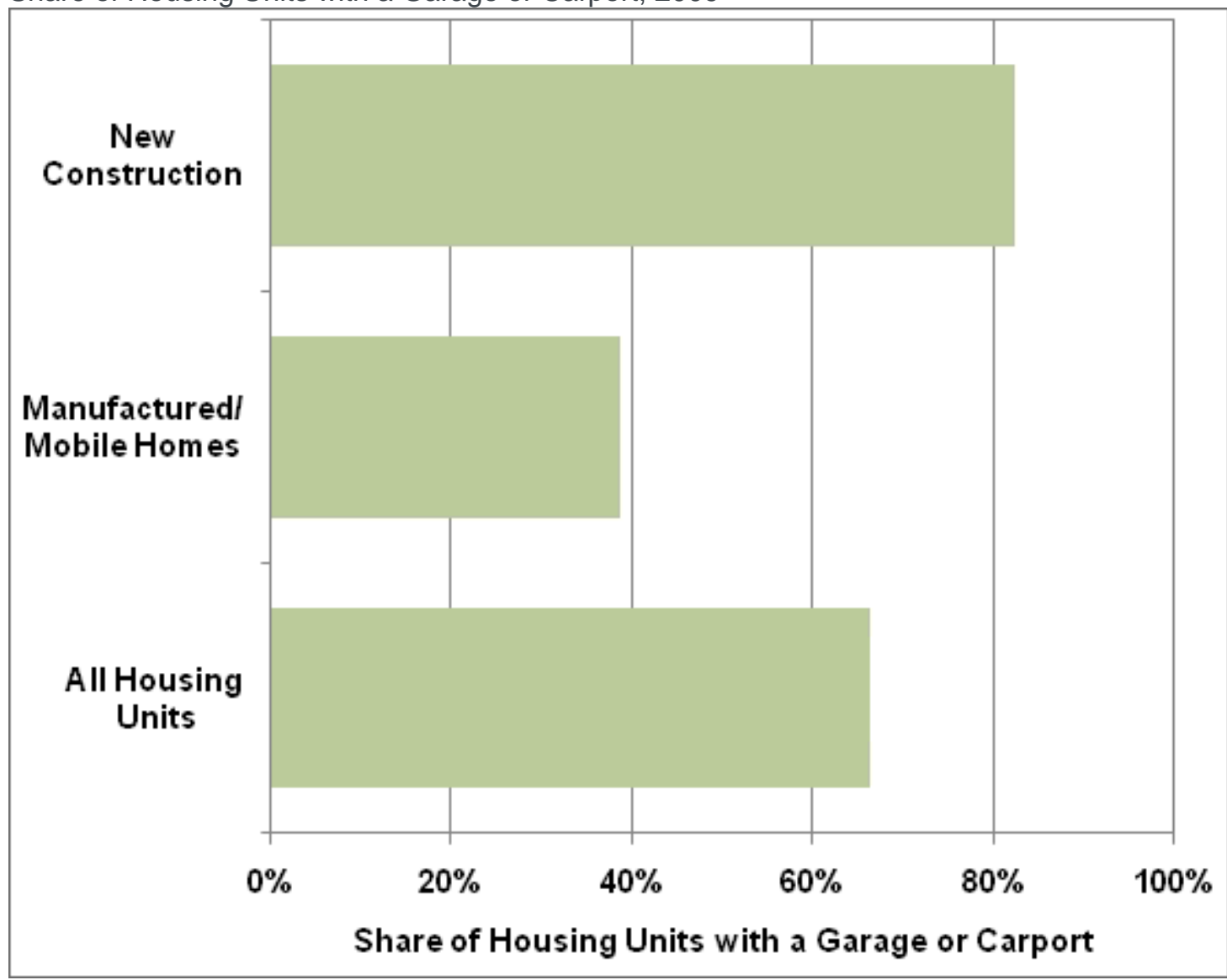

Note: New construction is any housing unit that is 4 years old or less.

A housing unit is a house, apartment, group of rooms, or single room occupied or intended for occupancy as separate living quarters. Group quarters, such as orphanages, nursing homes, dormitories, and military barracks are not included. 


\section{Supporting Information}

\begin{tabular}{|c|c|c|}
\hline \multicolumn{3}{|c|}{ Share of Housing Units with a Garage or Carport, 2009} \\
\hline & $\begin{array}{l}\text { Number of } \\
\text { housing units } \\
\text { (Millions) }\end{array}$ & $\begin{array}{c}\text { Share of units } \\
\text { with a garage or } \\
\text { carport }\end{array}$ \\
\hline $\begin{array}{l}\text { New Construction (4 } \\
\text { years old or less) }\end{array}$ & 4.8 & $82.3 \%$ \\
\hline $\begin{array}{l}\text { Manufactured/Mobile } \\
\text { Homes }\end{array}$ & 6.8 & $38.6 \%$ \\
\hline All Housing Units & 111.8 & $66.4 \%$ \\
\hline
\end{tabular}




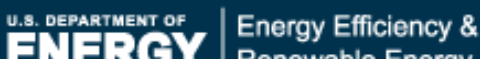 \\ Renewable Energy}

\section{Vehicle Technologies Program}

\section{Fact \#666: March 14, 2011 Survey Says Electric Vehicle Prices Are Key}

November/December 2010 surveys of 1,716 drivers and 123 automobile industry executives indicate that both groups believe a low electric vehicle price would motivate consumers to switch from a conventional vehicle to an electric-only vehicle (EV). More than half of the drivers surveyed also indicated that an extended vehicle range, the convenience of usage, and the availability of charging stations would motivate them to purchase an EV. The automobile industry executives however, placed higher emphasis on the price of oil and government incentives, with more than $70 \%$ of the executives naming those as important to consumers. Sustainability concerns and traffic congestion were chosen as a motivating factor more often by consumers than by the executives.

Responses to Survey on Electric Vehicles, November/December 2010

Consumers were asked: What would motivate you to switch from using a vehicle that currently runs on gasoline, diesel or hybrid to an electric-only vehicle?

Auto industry executives were shown the same question and asked to rate the importance consumers place on each choice. 


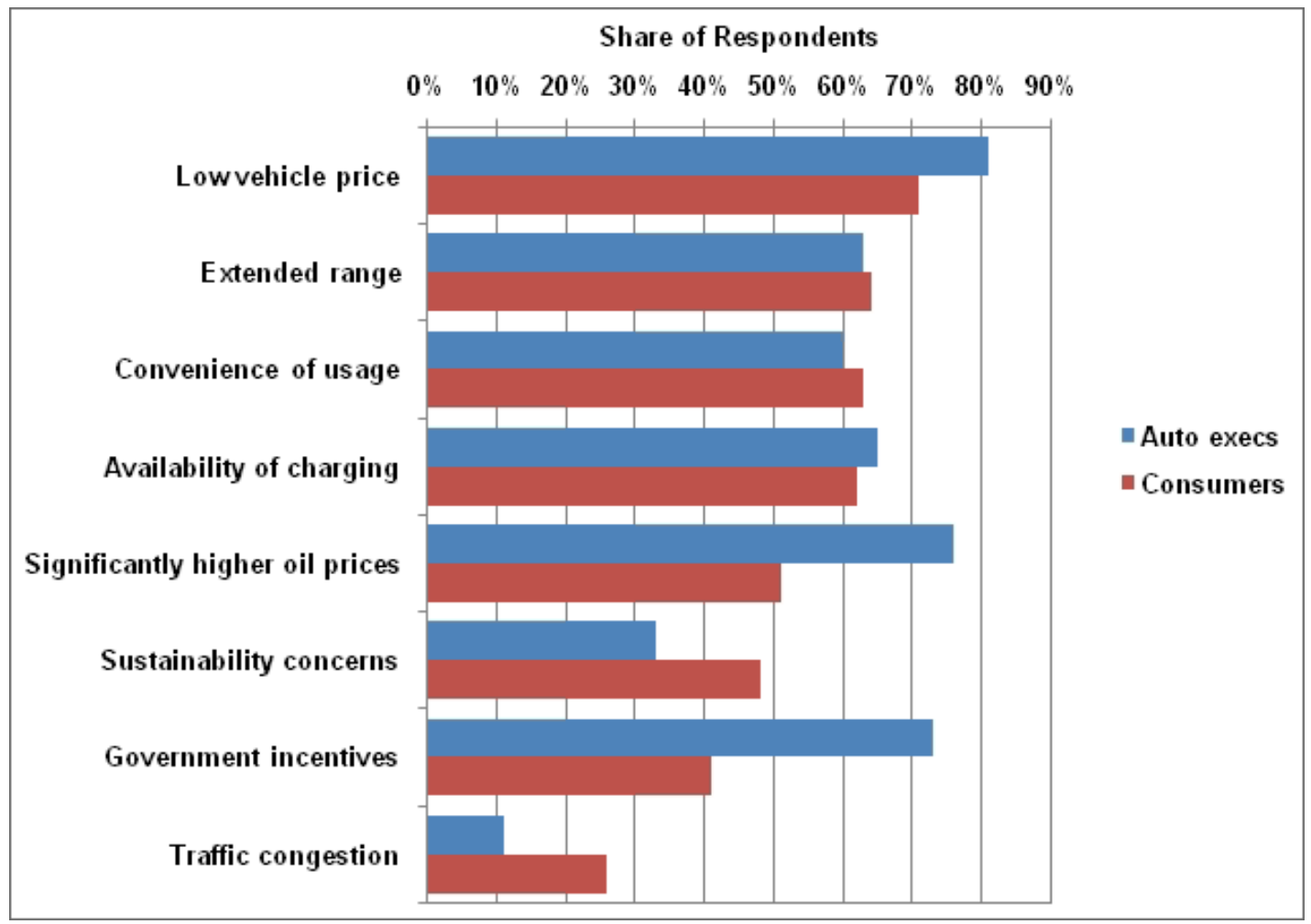




\section{Supporting Information}

Consumers were asked: What would motivate you to switch from using a vehicle that currently runs on gasoline, diesel or hybrid to an electric-only vehicle?

Auto industry executives were shown the same question and asked to rate the importance consumers place on each choice.

\begin{tabular}{|l|c|c|}
\hline & Consumers & $\begin{array}{c}\text { Auto } \\
\text { Executives }\end{array}$ \\
\hline $\begin{array}{l}\text { Innovative pricing models or } \\
\text { lower price overall }\end{array}$ & $71 \%$ & $81 \%$ \\
\hline $\begin{array}{l}\text { Extended reach or range of the } \\
\text { vehicles }\end{array}$ & $64 \%$ & $63 \%$ \\
\hline $\begin{array}{l}\text { Convenience of usage or } \\
\text { services }\end{array}$ & $63 \%$ & $60 \%$ \\
\hline $\begin{array}{l}\text { Availability of charging } \\
\text { infrastructure }\end{array}$ & $62 \%$ & $65 \%$ \\
\hline Significantly higher oil prices & $51 \%$ & $76 \%$ \\
\hline $\begin{array}{l}\text { Green image or sustainability } \\
\text { concerns }\end{array}$ & $48 \%$ & $33 \%$ \\
\hline $\begin{array}{l}\text { Government incentives or } \\
\text { regulations }\end{array}$ & $41 \%$ & $73 \%$ \\
\hline Traffic congestion & $26 \%$ & $11 \%$ \\
\hline Source: & & $73 \%$ \\
\hline
\end{tabular}

\section{Source:}

IBM Institute for Business Value, survey of nationally representative sample of 1,716 American drivers, December 2010; and interviews with 123 automobile industry executives, November 2010. 


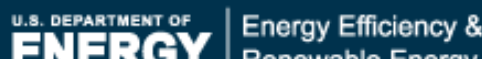 \\ Renewable Energy}

\section{Vehicle Technologies Program}

Fact \#667: March 21, 2011 Fuel Wasted in Traffic Congestion

The researchers at the Texas Transportation Institute have recently published new estimates of the effects of traffic congestion. The trend toward increased congestion eased in 2007 and 2008 with the downturn in the economy but began to rise again in 2009 along with economic activity. In 2009 , nearly 4 billion gallons of fuel were wasted due to traffic congestion; up 160 million gallons from 2008 but still lower than the peak of 4.4 billion gallons wasted in 2006 .

Total Fuel Wasted Due to Congestion 1982-2009

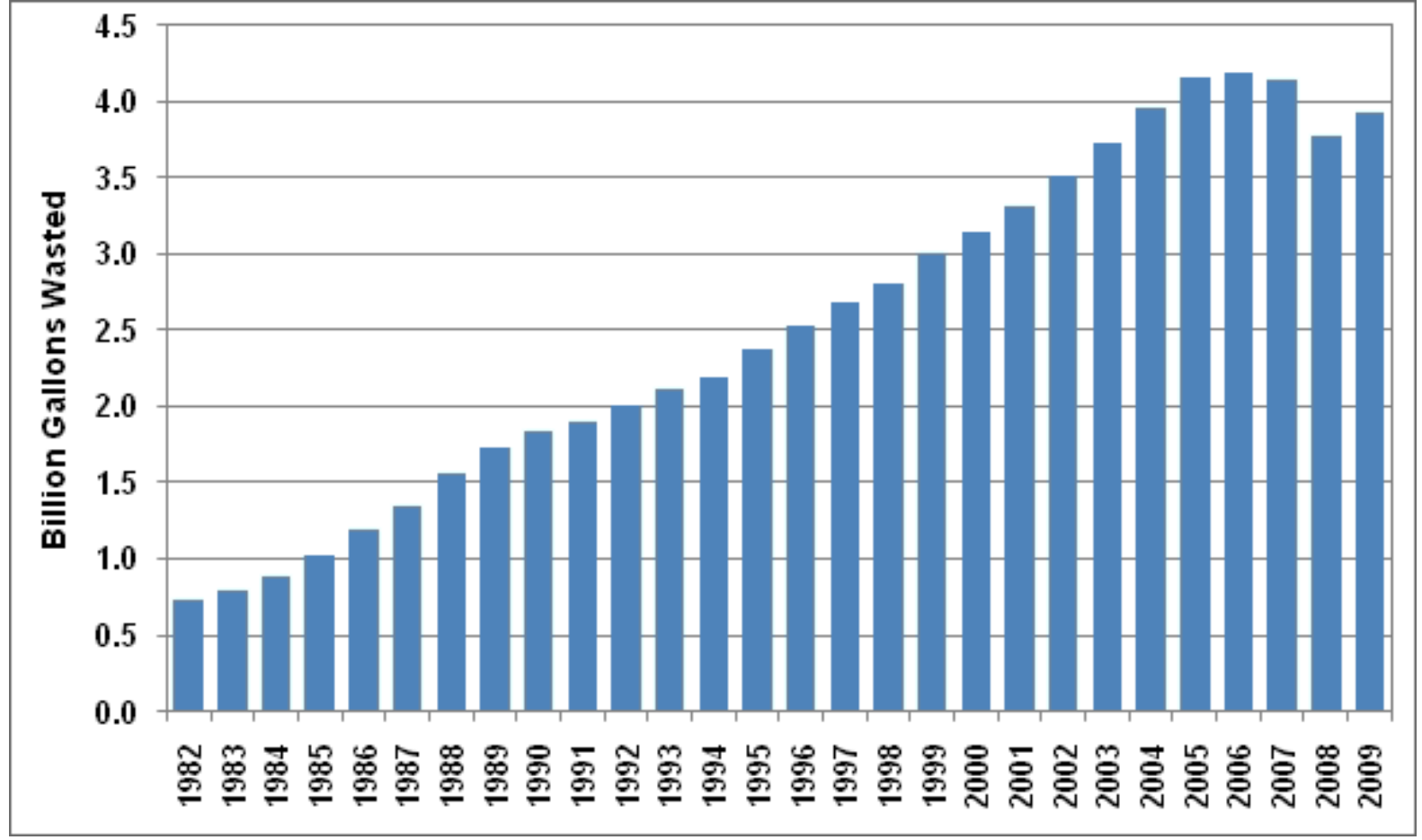




\section{Supporting Information}

\begin{tabular}{|c|c|c|c|}
\hline \multicolumn{4}{|c|}{$\begin{array}{l}\text { Total Fuel Wasted Due to Congestion, 1982- } \\
\qquad 2009\end{array}$} \\
\hline Year & $\begin{array}{l}\text { Fuel Wasted } \\
\text { (Billion } \\
\text { gallons) }\end{array}$ & Year & $\begin{array}{c}\text { Fuel Wasted } \\
\text { (Billion } \\
\text { gallons) }\end{array}$ \\
\hline 1982 & 0.73 & 1996 & 2.53 \\
\hline 1983 & 0.80 & 1997 & 2.68 \\
\hline 1984 & 0.88 & 1998 & 2.81 \\
\hline 1985 & 1.03 & 1999 & 3.01 \\
\hline 1986 & 1.20 & 2000 & 3.15 \\
\hline 1987 & 1.35 & 2001 & 3.31 \\
\hline 1988 & 1.56 & 2002 & 3.51 \\
\hline 1989 & 1.73 & 2003 & 3.72 \\
\hline 1990 & 1.84 & 2004 & 3.95 \\
\hline 1991 & 1.90 & 2005 & 4.15 \\
\hline 1992 & 2.01 & 2006 & 4.19 \\
\hline 1993 & 2.11 & 2007 & 4.14 \\
\hline 1994 & 2.19 & 2008 & 3.77 \\
\hline 1995 & 2.37 & 2009 & 3.93 \\
\hline
\end{tabular}




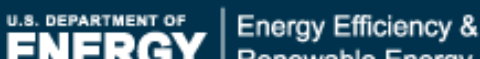 \\ Renewable Energy}

\section{Vehicle Technologies Program}

\section{Fact \#668: March 28, 2011 Time Wasted Due to Traffic Congestion}

According to the Texas Transportation Institute's latest study on traffic congestion, all urban areas have experienced increased congestion in the seventeen-year period from 1982 to 2009, but congestion has changed little from 1999 to 2009. As expected, traffic congestion is worse in very large urban areas, but congestion has grown in small areas, too. The amount of time commuters in private vehicles waste due to congestion has more than doubled from 1982 to 2009 for each of the urban areas shown.

Average Annual Hours Wasted Per Commuter Due to Congestion by Urban Area Size Category, 1982, 1999 and 2009

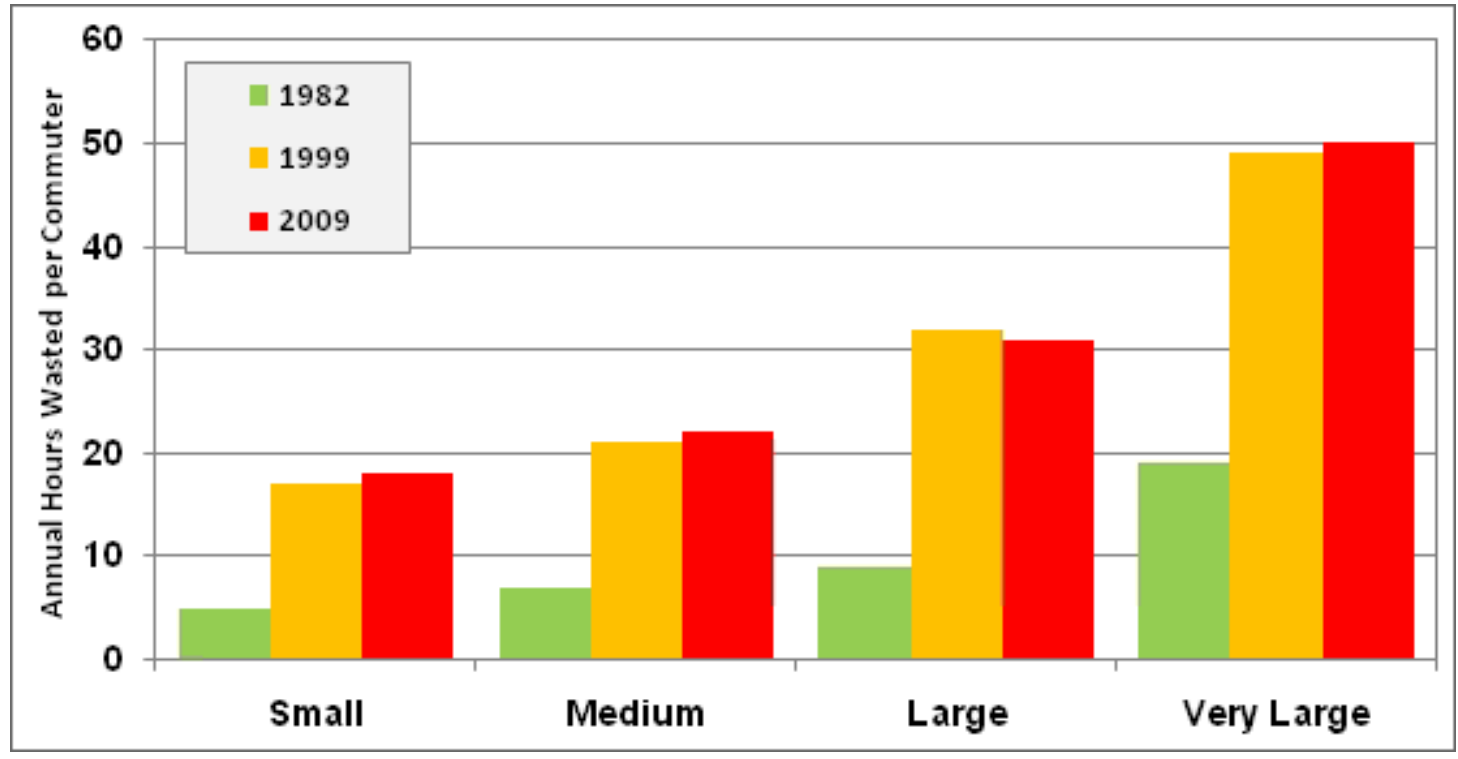

Note:

For commuters in private vehicles.

\section{Definitions:}

Small Urban Areas - Less than 500,000 population.

Medium Urban Areas - Over 500,000 but less than 1 million population.

Large Urban Areas - Over 1 million and less than 3 million population.

Very Large Urban Areas - Over 3 million population. 


\section{Supporting Information}

\begin{tabular}{|l|c|c|c|}
\hline \multicolumn{4}{|c|}{$\begin{array}{c}\text { Average Annual Hours Wasted Per } \\
\text { Commuter Due to Congestion by Urban }\end{array}$} \\
Area Size Category, 1982, 1999 and 2009
\end{tabular}




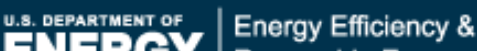 \\ Renewable Energy}

\section{Vehicle Technologies Program}

\section{Fact \#669: April 4, 2011}

\section{GM Sells More Vehicles in China than in the U.S.}

For the first time ever, General Motors (GM) sold more cars and trucks in China than in the United States. The demand in China grew by 29\% from 2009 to 2010, while demand in the U.S. grew $6 \%$. These data include cars, light trucks, and heavy trucks.

General Motors Vehicle Deliveries to China and the U.S., 2009-10

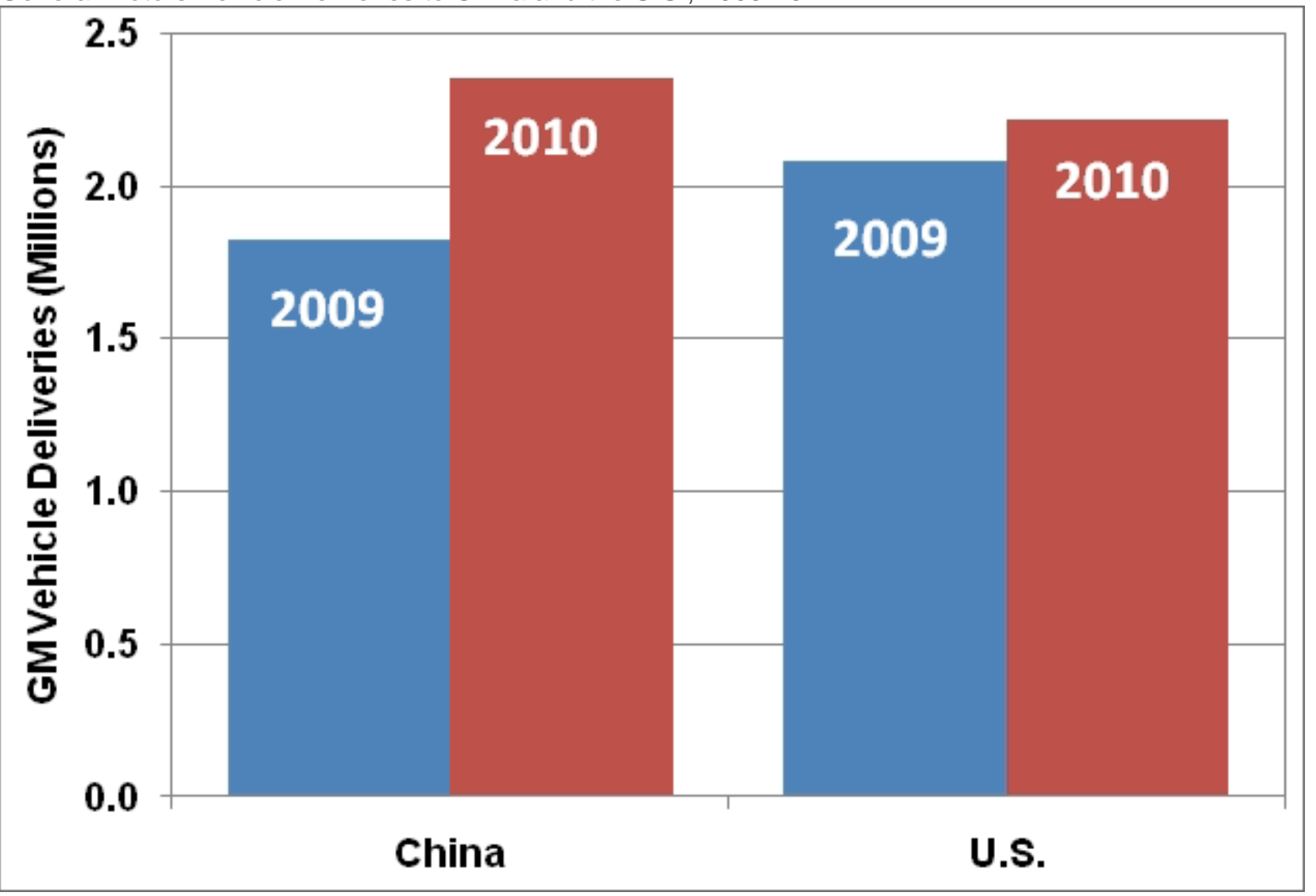




\section{Supporting Information}

\begin{tabular}{|c|c|c|c|}
\hline \multicolumn{4}{|c|}{$\begin{array}{l}\text { General Motors Vehicle Deliveries to China } \\
\text { and the U.S., } \\
2009-10\end{array}$} \\
\hline & $\begin{array}{l}2009 \\
\text { (Million } \\
\text { Vehicles) }\end{array}$ & $\begin{array}{l}2010 \\
\text { (Million } \\
\text { Vehicles) }\end{array}$ & $\begin{array}{l}\text { Percent } \\
\text { Change }\end{array}$ \\
\hline China & 1.83 & 2.35 & $29 \%$ \\
\hline U.S. & 2.08 & 2.22 & $6 \%$ \\
\hline \multicolumn{4}{|c|}{$\begin{array}{l}\text { Source: USA Today, "GM sells more vehicle in } \\
\text { China than in U.S.," January 24, 2011. [Original } \\
\text { data source: General Motors.] }\end{array}$} \\
\hline
\end{tabular}




\section{U.s. ofpartment of | Energy Efficiency \& \\ Renewable Energy}

\section{Vehicle Technologies Program}

Fact \#670: April 11, 2011

Vehicle-Miles of Travel Rises in 2010

The preliminary estimates from the Federal Highway Administration show that vehiclemiles of travel (VMT) increased slightly in 2010 over the previous year, but have not surpassed the peak of 3.03 trillion miles in 2007. Total U.S. VMT declined during the economic downturns in the mid-70's, early 80's, and in 2008.

Total Vehicle-Miles of Travel, 1970 - 2010

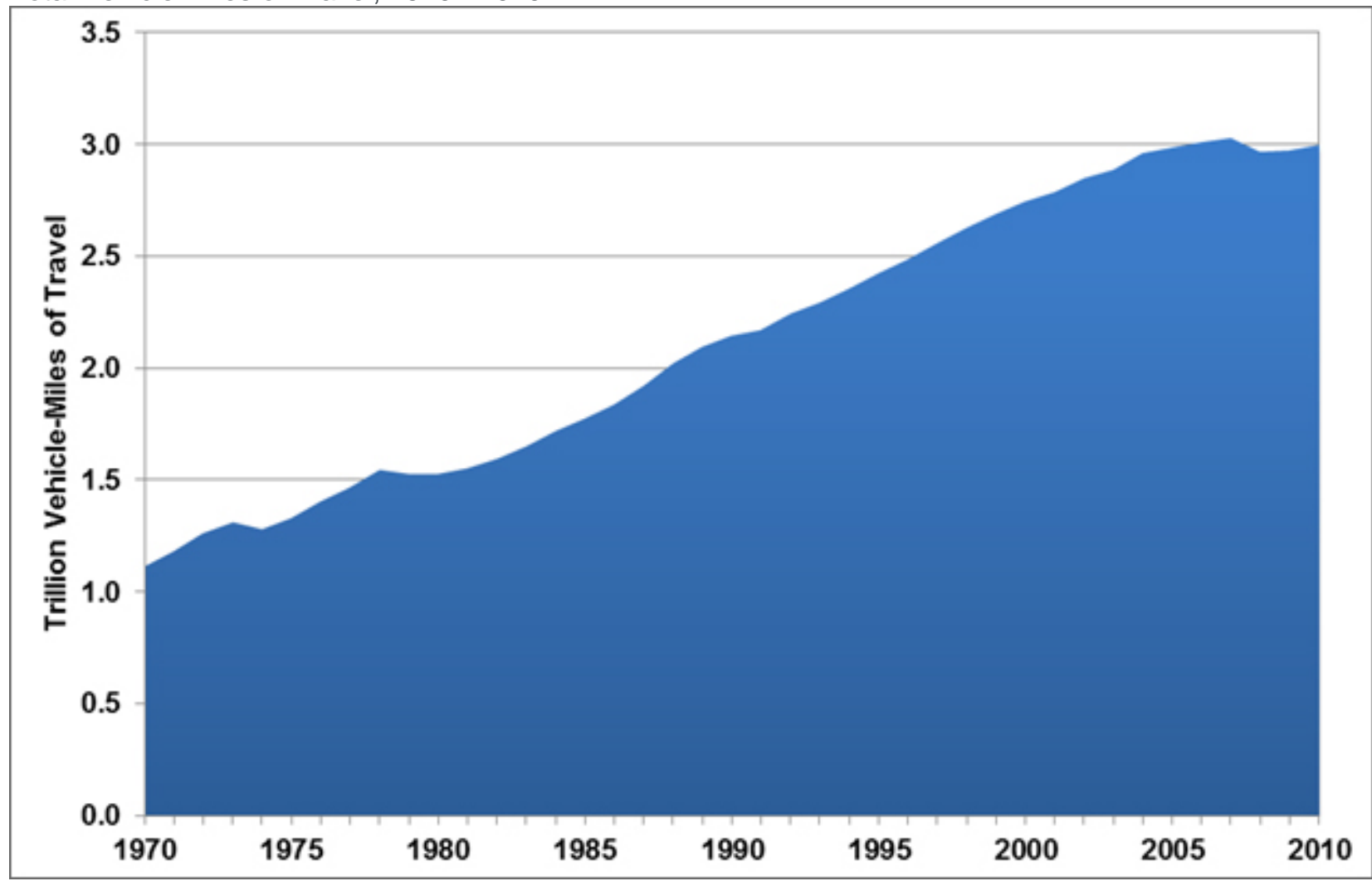




\section{Supporting Information}

\begin{tabular}{|c|c|c|c|}
\hline \multicolumn{4}{|c|}{ Total Vehicle-Miles of Travel, $1970-2010$} \\
\hline Year & Trillion Miles & Year & Trillion Miles \\
\hline 1970 & 1.11 & 1991 & 2.17 \\
\hline 1971 & 1.18 & 1992 & 2.25 \\
\hline 1972 & 1.26 & 1993 & 2.30 \\
\hline 1973 & 1.31 & 1994 & 2.36 \\
\hline 1974 & 1.28 & 1995 & 2.42 \\
\hline 1975 & 1.33 & 1996 & 2.49 \\
\hline 1976 & 1.40 & 1997 & 2.56 \\
\hline 1977 & 1.47 & 1998 & 2.63 \\
\hline 1978 & 1.54 & 1999 & 2.69 \\
\hline 1979 & 1.53 & 2000 & 2.75 \\
\hline 1980 & 1.53 & 2001 & 2.79 \\
\hline 1981 & 1.56 & 2002 & 2.86 \\
\hline 1982 & 1.60 & 2003 & 2.89 \\
\hline 1983 & 1.65 & 2004 & 2.96 \\
\hline 1984 & 1.72 & 2005 & 2.99 \\
\hline 1985 & 1.77 & 2006 & 3.01 \\
\hline 1986 & 1.83 & 2007 & 3.03 \\
\hline 1987 & 1.92 & 2008 & 2.97 \\
\hline 1988 & 2.03 & 2009 & 2.98 \\
\hline 1989 & 2.10 & 2010 & 3.00 \\
\hline 1990 & 2.14 & & \\
\hline \multicolumn{4}{|c|}{$\begin{array}{l}\text { Sources: 1970-2008: U.S. Department of Transportation, Federal } \\
\text { Highway Administration, Highway Statistics Series. } \\
\text { 2009-2010: U.S. Department of Transportation, Federal Highway } \\
\text { Administration, Traffic Volume Trends. }\end{array}$} \\
\hline
\end{tabular}




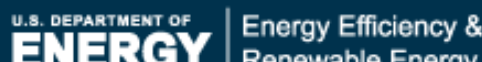 \\ Renewable Energy}

\section{Vehicle Technologies Program}

\section{Fact \#671: April 18, 2011 Average Truck Speeds}

The Federal Highway Administration studies traffic volume and flow on major truck routes by tracking more than 500,000 trucks. The average speed of trucks on selected interstate highways is between 50 and 60 miles per hour ( $\mathrm{mph}$ ). The average operating speed of trucks is typically below $55 \mathrm{mph}$ in major urban areas, border crossings, and in mountainous terrain. The difference in average speed between peak traffic times and non-peak hours can be as much as $1.5 \mathrm{mph}(\mathrm{I}-45)$ or as little as $0.2 \mathrm{mph}(\mathrm{I}-81)$.

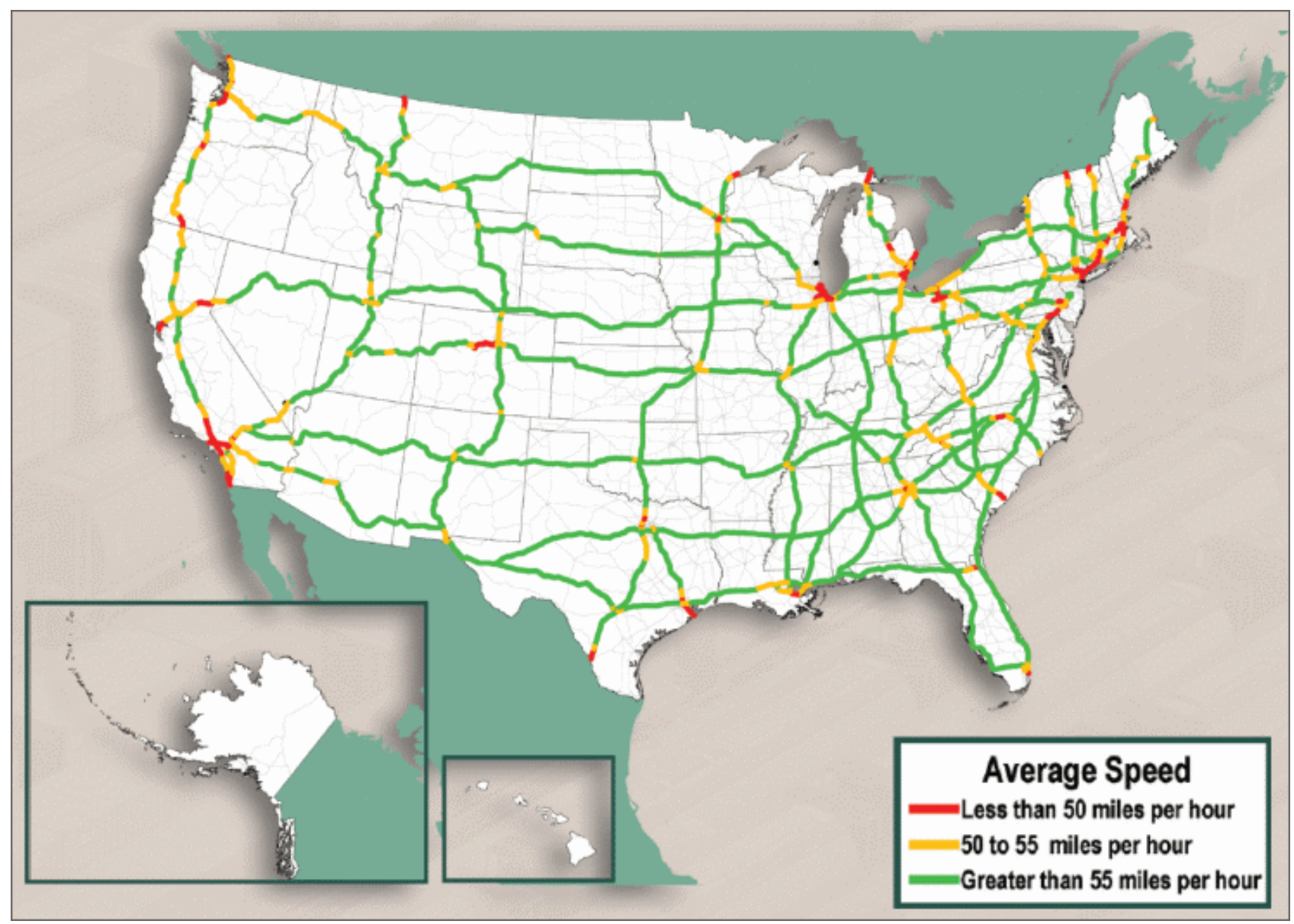




\section{Supporting Information}

\begin{tabular}{|c|c|c|c|c|}
\hline \multicolumn{5}{|c|}{ Average Truck Speeds on Selected Interstate Highways } \\
\hline \multirow{2}{*}{$\begin{array}{c}\text { Interstate } \\
\text { Route }\end{array}$} & $\begin{array}{c}\text { Average } \\
\text { Operating } \\
\text { Speed }\end{array}$ & $\begin{array}{c}\text { Peak } \\
\text { Period } \\
\text { Average } \\
\text { Speed }\end{array}$ & $\begin{array}{l}\text { Non-Peak } \\
\text { Period } \\
\text { Average } \\
\text { Speed }\end{array}$ & $\begin{array}{c}\text { Difference } \\
\text { between Non- } \\
\text { peak and } \\
\text { Peak Speeds }\end{array}$ \\
\hline & \multicolumn{4}{|c|}{ (Miles per Hour) } \\
\hline 5 & 52.8 & 52.0 & 53.1 & 1.1 \\
\hline 10 & 57.4 & 56.7 & 57.6 & 0.9 \\
\hline 15 & 56.7 & 56.2 & 56.9 & 0.7 \\
\hline 20 & 59.2 & 58.8 & 59.3 & 0.5 \\
\hline 24 & 57.2 & 56.6 & 57.4 & 0.8 \\
\hline 25 & 58.9 & 58.5 & 59.3 & 0.8 \\
\hline 26 & 53.7 & 53.3 & 54.6 & 1.3 \\
\hline 35 & 56.8 & 55.9 & 57.0 & 1.1 \\
\hline 40 & 58.6 & 58.3 & 58.8 & 0.5 \\
\hline 45 & 54.9 & 53.9 & 55.4 & 1.5 \\
\hline 55 & 57.0 & 56.8 & 57.2 & 0.4 \\
\hline 65 & 57.9 & 57.3 & 58.2 & 0.9 \\
\hline 70 & 56.8 & 56.5 & 57.1 & 0.6 \\
\hline 75 & 56.7 & 56.1 & 57.0 & 0.9 \\
\hline 76 & 54.5 & 54.5 & 54.8 & 0.3 \\
\hline 77 & 54.7 & 54.3 & 55.1 & 0.8 \\
\hline 80 & 57.7 & 57.4 & 57.9 & 0.5 \\
\hline 81 & 56.6 & 56.6 & 56.8 & 0.2 \\
\hline 84 & 54.2 & 53.3 & 54.8 & 1.5 \\
\hline 85 & 57.3 & 56.5 & 57.4 & 0.9 \\
\hline
\end{tabular}




\begin{tabular}{|c|c|c|c|c|}
\hline \multicolumn{5}{|c|}{ Average Truck Speeds on Selected Interstate Highways } \\
\hline \multirow[t]{2}{*}{$\begin{array}{c}\text { Interstate } \\
\text { Route }\end{array}$} & $\begin{array}{l}\text { Average } \\
\text { Operating } \\
\text { Speed }\end{array}$ & $\begin{array}{c}\text { Peak } \\
\text { Period } \\
\text { Average } \\
\text { Speed }\end{array}$ & $\begin{array}{c}\text { Non-Peak } \\
\text { Period } \\
\text { Average } \\
\text { Speed }\end{array}$ & $\begin{array}{c}\text { Difference } \\
\text { between Non- } \\
\text { peak and } \\
\text { Peak Speeds }\end{array}$ \\
\hline & \multicolumn{4}{|c|}{ (Miles per Hour) } \\
\hline 87 & 54.1 & 53.8 & 54.5 & 0.7 \\
\hline 90 & 57.1 & 56.8 & 57.4 & 0.6 \\
\hline 91 & 53.4 & 52.9 & 54.2 & 1.3 \\
\hline 94 & 56.7 & 56.2 & 56.8 & 0.6 \\
\hline 95 & 56.2 & 55.2 & 56.3 & 1.1 \\
\hline \multicolumn{5}{|c|}{$\begin{array}{l}\text { Source: U.S. Department of Transportation, Federal Highway } \\
\text { Administration, Office of Freight Management and Operations, Freight } \\
\text { Facts and Figures 2010, Figure 3-13 and Table 3-8. }\end{array}$} \\
\hline
\end{tabular}




\section{U.s. oepartment of | Energy Efficiency \& \\ Renewable Energy}

\section{Vehicle Technologies Program}

\section{Fact \#672: April 25, 2011 Freight Gateways in the U.S.}

The top 25 places (in terms of freight value) that freight is shipped into and out of the U.S. are listed on the map below. Import values are shown by the gray bar, while export values are shown by the blue bar. Of the top 25 places, twelve are water ports, five are land-border crossings, and eight are air gateways. In most cases, the value of the imports exceeds the exports.

Top 25 Foreign-Trade Freight Gateways by Value, 2008

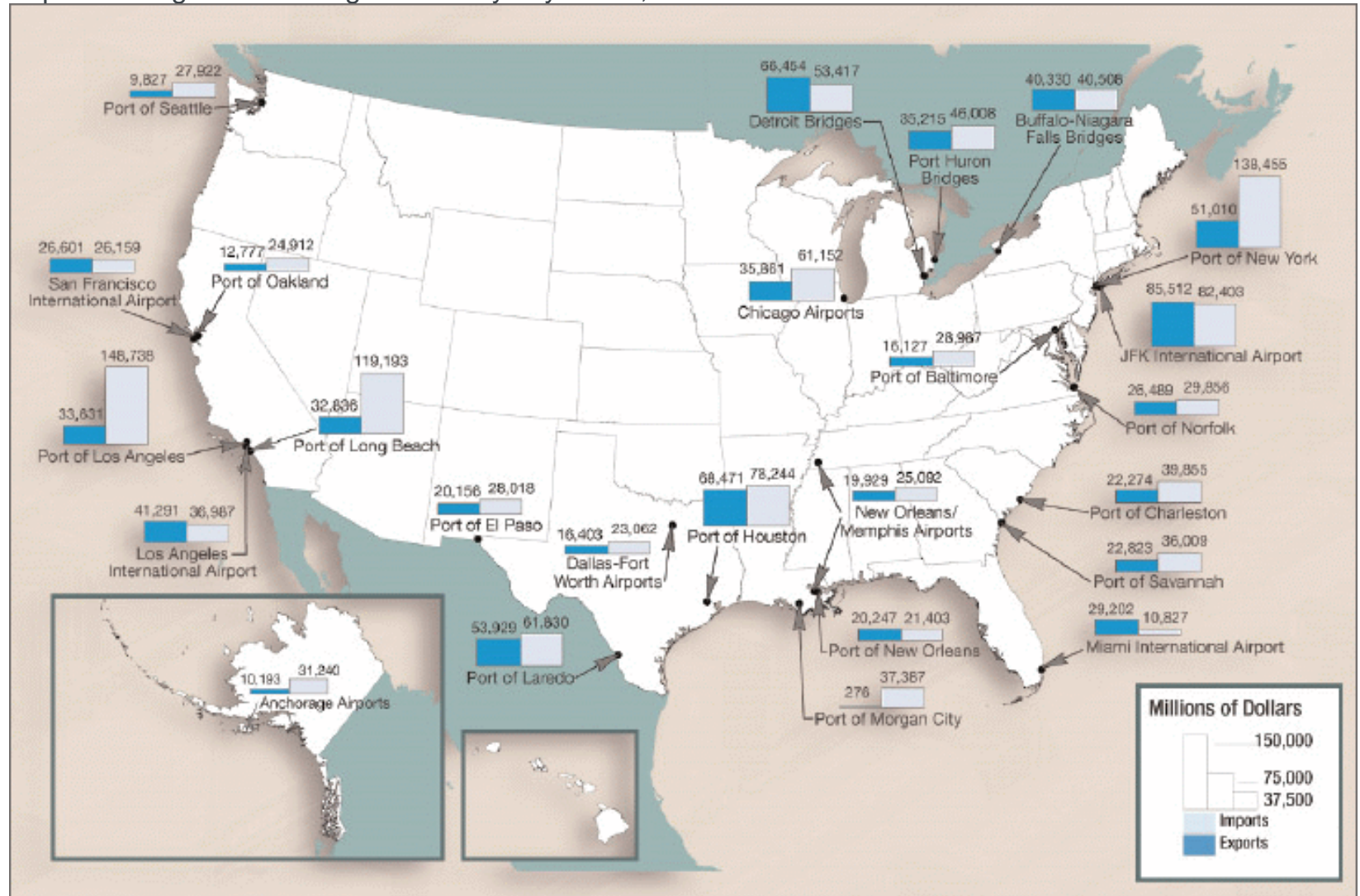




\section{Supporting Information}

\begin{tabular}{|c|c|c|c|c|c|}
\hline \multicolumn{6}{|c|}{ Top 25 Foreign-Trade Gateways by Value, 2008} \\
\hline \multirow{2}{*}{$\begin{array}{l}\text { Gateway } \\
\text { Route }\end{array}$} & \multirow[b]{2}{*}{ Type } & \multirow[b]{2}{*}{ Rank } & Exports & Imports & Total \\
\hline & & & \multicolumn{3}{|c|}{ (Billion U.S. Dollars) } \\
\hline New York, NY & Water & 1 & 51 & 138.5 & 189.5 \\
\hline Los Angeles, CA & Water & 2 & 33.6 & 148.7 & 182.4 \\
\hline $\begin{array}{l}\text { John F. Kennedy } \\
\text { International, NY }\end{array}$ & Air & 3 & 85.5 & 82.4 & 167.9 \\
\hline Long Beach, CA & Water & 4 & 32.8 & 119.2 & 152 \\
\hline Houston, TX & Water & 5 & 68.5 & 78.2 & 146.7 \\
\hline Detroit, MI & Land & 6 & 66.5 & 53.7 & 120.2 \\
\hline Laredo, TX & Land & 7 & 53.9 & 61.8 & 115.8 \\
\hline Chicago, IL & Air & 8 & 35.9 & 61.2 & 97 \\
\hline Port Huron, MI & Land & 9 & 35.2 & 46 & 81.2 \\
\hline Buffalo-Niagara Falls, NY & Land & 10 & 40.3 & 40.5 & 80.8 \\
\hline $\begin{array}{l}\text { Los Angeles International, } \\
\text { CA }\end{array}$ & Air & 11 & 41.3 & 37 & 78.3 \\
\hline Charleston, SC & Water & 12 & 22.3 & 39.9 & 62.1 \\
\hline Savannah, GA & Water & 13 & 22.8 & 36 & 58.8 \\
\hline Norfolk, VA & Water & 14 & 26.5 & 29.9 & 56.3 \\
\hline $\begin{array}{l}\text { San Francisco International, } \\
\text { CA }\end{array}$ & Air & 15 & 26.6 & 26.2 & 52.8 \\
\hline El Paso, TX & Land & 16 & 20.2 & 28 & 48.2 \\
\hline Baltimore, MD & Water & 17 & 16.1 & 29 & 45.1 \\
\hline New Orleans, LA & Air & 18 & 19.9 & 25.1 & 45 \\
\hline New Orleans, LA & Water & 19 & 20.2 & 21.4 & 41.7 \\
\hline Anchorage, AK & Air & 20 & 10.2 & 31.2 & 41.4 \\
\hline Miami International, FL & Air & 21 & 29.2 & 10.8 & 40 \\
\hline Dallas-Fort Worth, TX & Air & 22 & 16.4 & 23.1 & 39.5 \\
\hline Seattle, WA & Water & 23 & 9.8 & 27.9 & 37.7 \\
\hline
\end{tabular}




\section{Top 25 Foreign-Trade Gateways by Value, 2008}

\begin{tabular}{|l|l|c|c|c|c|}
\multicolumn{1}{|c|}{$\begin{array}{c}\text { Gateway } \\
\text { Route }\end{array}$} & Type & Rank & \multicolumn{2}{|c|}{ (Billion U.S. Dollars) } \\
\hline Oakland, CA & Water & 24 & 12.8 & 24.9 & 37.7 \\
\hline $\begin{array}{l}\text { Morgan City, } \\
\text { LA }\end{array}$ & Water & 25 & 0.3 & 37.4 & 37.7 \\
\hline
\end{tabular}

Notes: Data for all air gateways include a low level (generally less than $2 \%$ $3 \%$ of the total value) of small user-fee airports located in the same region. Air gateways not identified by airport name (e.g., Chicago, IL, and others) include major airport(s) in that geographic area in addition to small regional airports. Due to Census Bureau confidentiality regulations, data for courier operations are included in the airport totals for JFK International Airport, New Orleans, Los Angeles, Cleveland, Chicago, Miami, and Anchorage.

Source: U.S. Department of Transportation, Federal Highway Administration, Office of Freight Management and Operations, Freight Facts and Figures 2010, Figure 2-2. 


\section{u.s. Depaatment of | Energy Efficiency \& \\ Renewable Energy}

\section{Vehicle Technologies Program}

\section{Fact \#673: May 2, 2011}

\section{U.S. Trade Balance for Transportation Vehicles}

Motor vehicles, aircraft, ships, and rail locomotives are imported to and exported from the U.S. The trade balance (exports minus imports) shows that the U.S. imports more motor vehicles and parts than it exports. However, aircraft, spacecraft, and parts are exported more often than imported. Comparatively few water and rail vehicles are traded, thus the trade balance for those modes is small. Transportation vehicles make up $12 \%$ of the total of all commodities traded.

U.S. Trade Balance for Transportation Vehicles, 2009

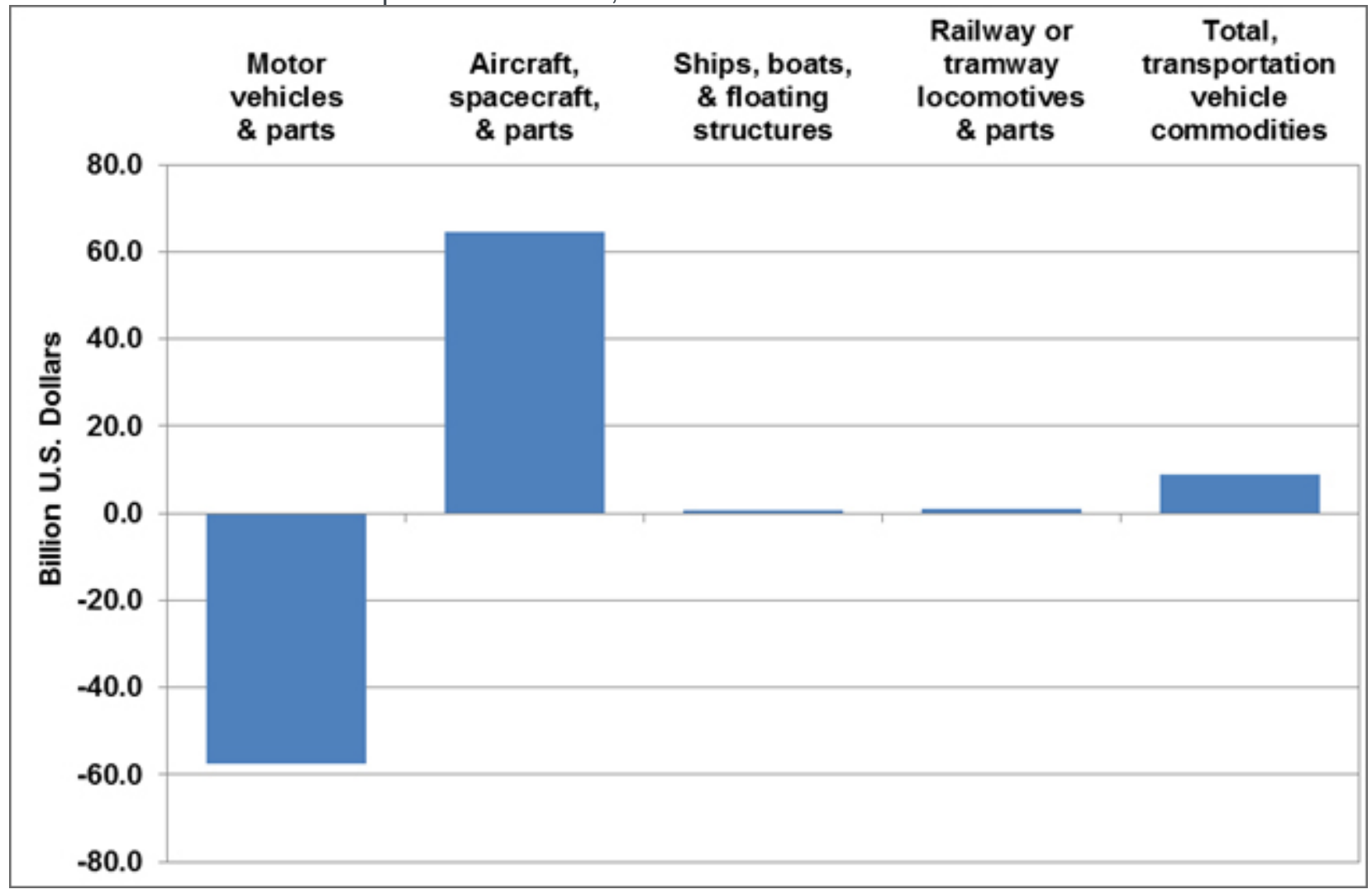




\section{Supporting Information}

\begin{tabular}{|l|c|c|c|c|}
\hline \multicolumn{4}{|c|}{ U.S. Trade in Transportation Vehicle Commodities, 2009} \\
(Billion U.S. dollars)
\end{tabular}




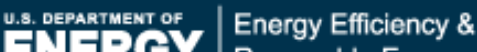 \\ Renewable Energy}

\section{Vehicle Technologies Program}

\section{Fact \#674: May 9, 2011}

\section{Petroleum Trade Balance}

The trade balance (exports minus imports) for petroleum has been negative for more than a quarter of a century, meaning that the U.S. imports more petroleum than it exports. The largest petroleum trade deficit was in 2008 , when the U.S. imported $\$ 449.8$ billion and exported only $\$ 61.6$ billion.

Petroleum Trade Balance, 1970-2010

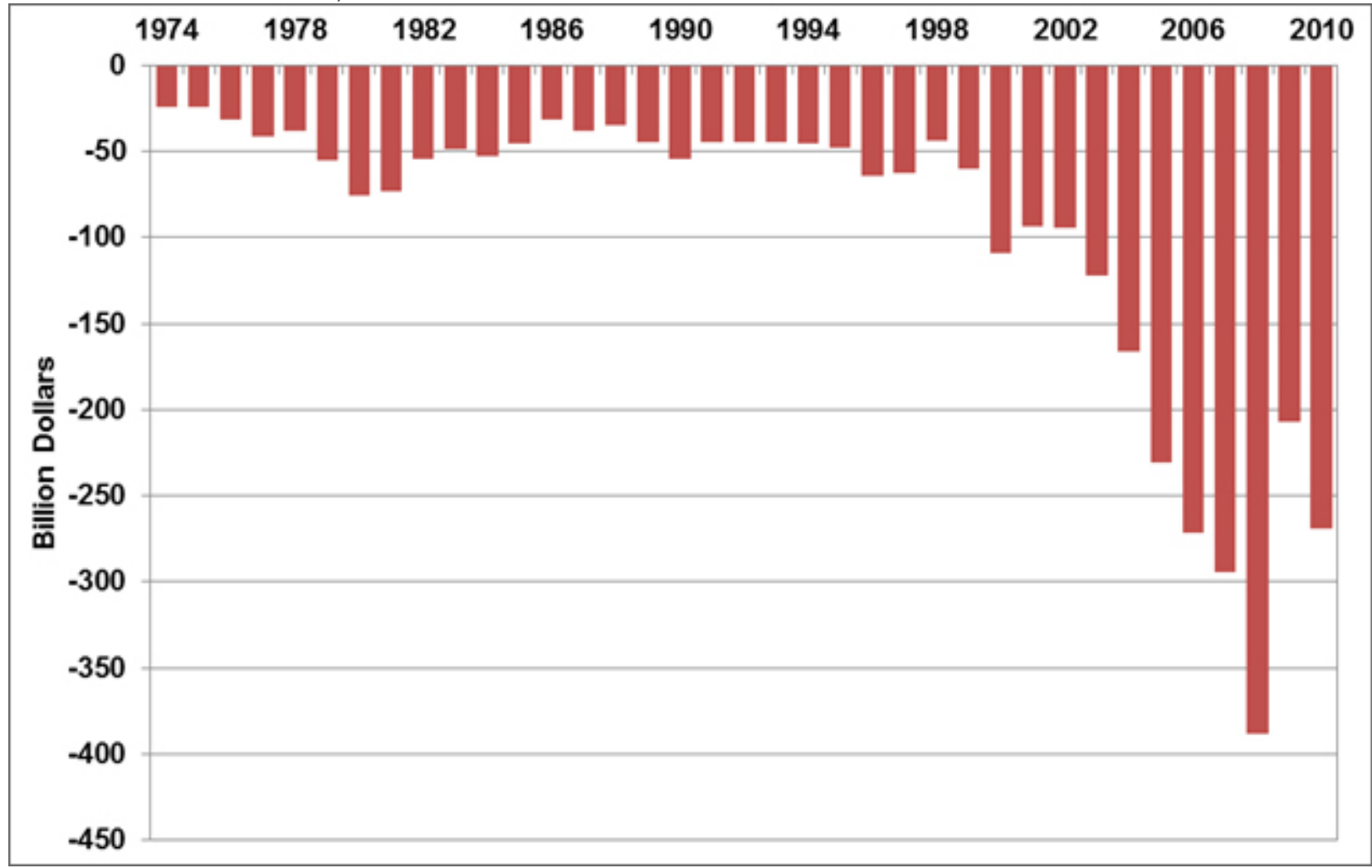

Note: Trade balance $=$ exports minus imports. Includes crude oil, petroleum preparations, liquefied propane and butane, and other mineral fuels. 


\section{Supporting Information}

\begin{tabular}{|c|c|c|c|}
\hline \multicolumn{4}{|c|}{ Petroleum Exports, Imports, and Trade Balance, 1970-2010 } \\
\hline \multirow{2}{*}{$\begin{array}{l}\text { Year } \\
\text { Route }\end{array}$} & $\begin{array}{l}\text { Petroleum } \\
\text { Exports }\end{array}$ & $\begin{array}{l}\text { Petroleum } \\
\text { Imports }\end{array}$ & $\begin{array}{l}\text { Petroleum } \\
\text { Balance }\end{array}$ \\
\hline & \multicolumn{3}{|c|}{ (Billion Dollars) } \\
\hline 1974 & 0.792 & 24.668 & -23.876 \\
\hline 1975 & 0.907 & 25.197 & -24.289 \\
\hline 1976 & 0.998 & 32.226 & -31.228 \\
\hline 1977 & 1.276 & 42.368 & -41.093 \\
\hline 1978 & 1.561 & 39.526 & -37.965 \\
\hline 1979 & 1.914 & 56.715 & -54.801 \\
\hline 1980 & 2.833 & 78.637 & -75.803 \\
\hline 1981 & 3.696 & 76.659 & -72.963 \\
\hline 1982 & 5.947 & 60.458 & -54.511 \\
\hline 1983 & 4.557 & 53.217 & -48.659 \\
\hline 1984 & 4.470 & 56.924 & -52.454 \\
\hline 1985 & 4.707 & 50.475 & -45.768 \\
\hline 1986 & 3.640 & 35.142 & -31.503 \\
\hline 1987 & 3.922 & 42.285 & -38.363 \\
\hline 1988 & 3.693 & 38.787 & -35.094 \\
\hline 1989 & 5.021 & 49.704 & -44.683 \\
\hline 1990 & 6.901 & 61.583 & -54.682 \\
\hline 1991 & 6.954 & 51.350 & -44.396 \\
\hline 1992 & 6.412 & 51.217 & -44.805 \\
\hline 1993 & 6.215 & 51.046 & -44.831 \\
\hline 1994 & 5.659 & 50.835 & -45.176 \\
\hline 1995 & 6.321 & 54.368 & -48.047 \\
\hline 1996 & 7.984 & 72.022 & -64.038 \\
\hline 1997 & 8.592 & 71.152 & -62.560 \\
\hline 1998 & 6.574 & 50.264 & -43.690 \\
\hline 1999 & 7.118 & 67.173 & -60.055 \\
\hline
\end{tabular}




\begin{tabular}{|c|c|c|c|}
\hline \multirow{2}{*}{$\begin{array}{c}\text { Petroleum Exports, Imports, and Trade Balance, 1970-2010 } \\
\text { Year } \\
\text { Route }\end{array}$} & $\begin{array}{c}\text { Petroleum } \\
\text { Exports }\end{array}$ & $\begin{array}{c}\text { Petroleum } \\
\text { Imports }\end{array}$ & $\begin{array}{c}\text { Petroleum } \\
\text { Balance }\end{array}$ \\
\hline 2000 & 10.192 & 119.251 & -109.059 \\
\hline 2001 & 8.868 & 102.747 & -93.879 \\
\hline 2002 & 8.569 & 102.663 & -94.094 \\
\hline 2003 & 10.209 & 132.433 & -122.224 \\
\hline 2004 & 13.130 & 179.266 & -166.136 \\
\hline 2005 & 19.155 & 250.068 & -230.913 \\
\hline 2006 & 28.171 & 299.714 & -271.543 \\
\hline 2007 & 33.293 & 327.620 & -294.327 \\
\hline 2008 & 61.695 & 449.847 & -388.152 \\
\hline 2009 & 44.509 & 251.833 & -207.324 \\
\hline 2010 & 64.540 & 333.354 & -268.814 \\
\hline
\end{tabular}

Source: U.S. Department of Energy, Energy Information Administration, Monthly Energy Review, Table 1.5. 


\section{U.s. ofpartment of | Energy Efficiency \& \\ Renewable Energy}

\section{Vehicle Technologies Program}

Fact \#675: May 16, 2011

Gasoline Prices by Region, May 2, 2011

The West Coast region paid the highest prices for gasoline averaging $\$ 4.14$ for a gallon of regular gasoline while the Rocky Mountain region paid the least at $\$ 3.70$ per gallon. The southern states, including the Gulf Coast Region and the Lower Atlantic region, paid less than the states in the Midwest, Central Atlantic and New England regions where gasoline was about $\$ 4.00$ per gallon.

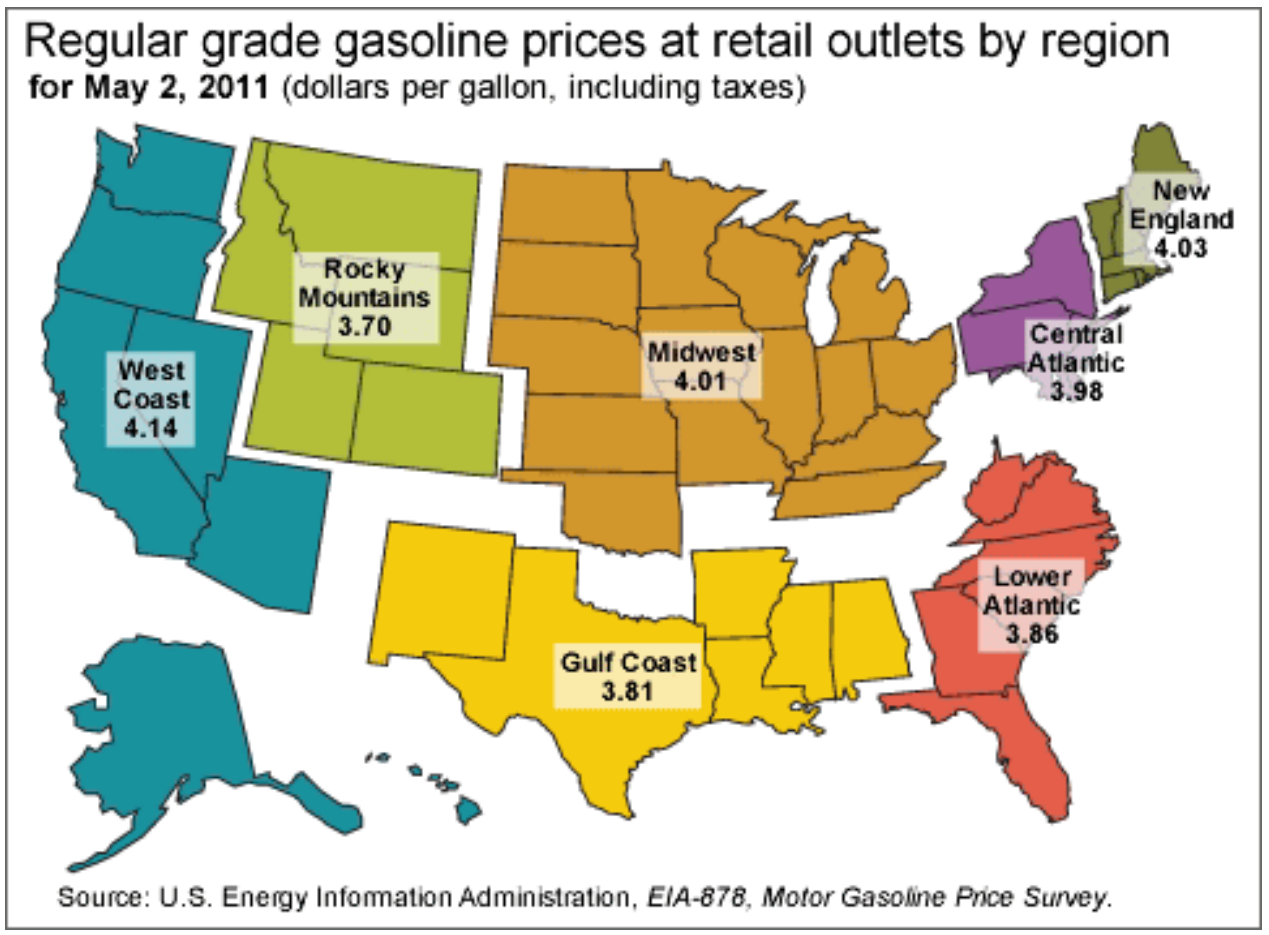




\section{Supporting Information}

\begin{tabular}{|l|c|}
\hline \multicolumn{2}{|c|}{$\begin{array}{c}\text { Regular Grade Gasoline Prices by Region, } \\
\text { May 2, } 2011\end{array}$} \\
\hline Region & $\begin{array}{c}\text { Price per gallon } \\
\text { including taxes }\end{array}$ \\
\hline New England & $\$ 4.14$ \\
\hline Midwest & $\$ 4.03$ \\
\hline Central Atlantic & $\$ 4.01$ \\
\hline Lower Atlantic & $\$ 3.98$ \\
\hline Gulf Coast & $\$ 3.86$ \\
\hline Rocky Mountains & $\$ 3.81$ \\
\hline Source: U.S. Energy Information Administration, \\
EIA-878, Motor Gasoline Price Survey. \\
\hline
\end{tabular}




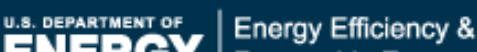 \\ Renewable Energy}

\section{Vehicle Technologies Program}

Fact \#676: May 23, 2011

U.S. Refiners Produce about 19 Gallons of Gasoline from a Barrel of Oil

A standard U.S. barrel contains 42 gallons of crude oil which yields about 44 gallons of petroleum products. The additional 2 gallons of petroleum products come from refiner gains which result in an additional $6 \%$ of product. As shown in the figure below, a little more than 19 gallons of gasoline and 10 gallons of diesel fuel are produced from a barrel of crude oil by U.S. refiners. Other products such as jet fuel and heating oil make up the remaining one third.

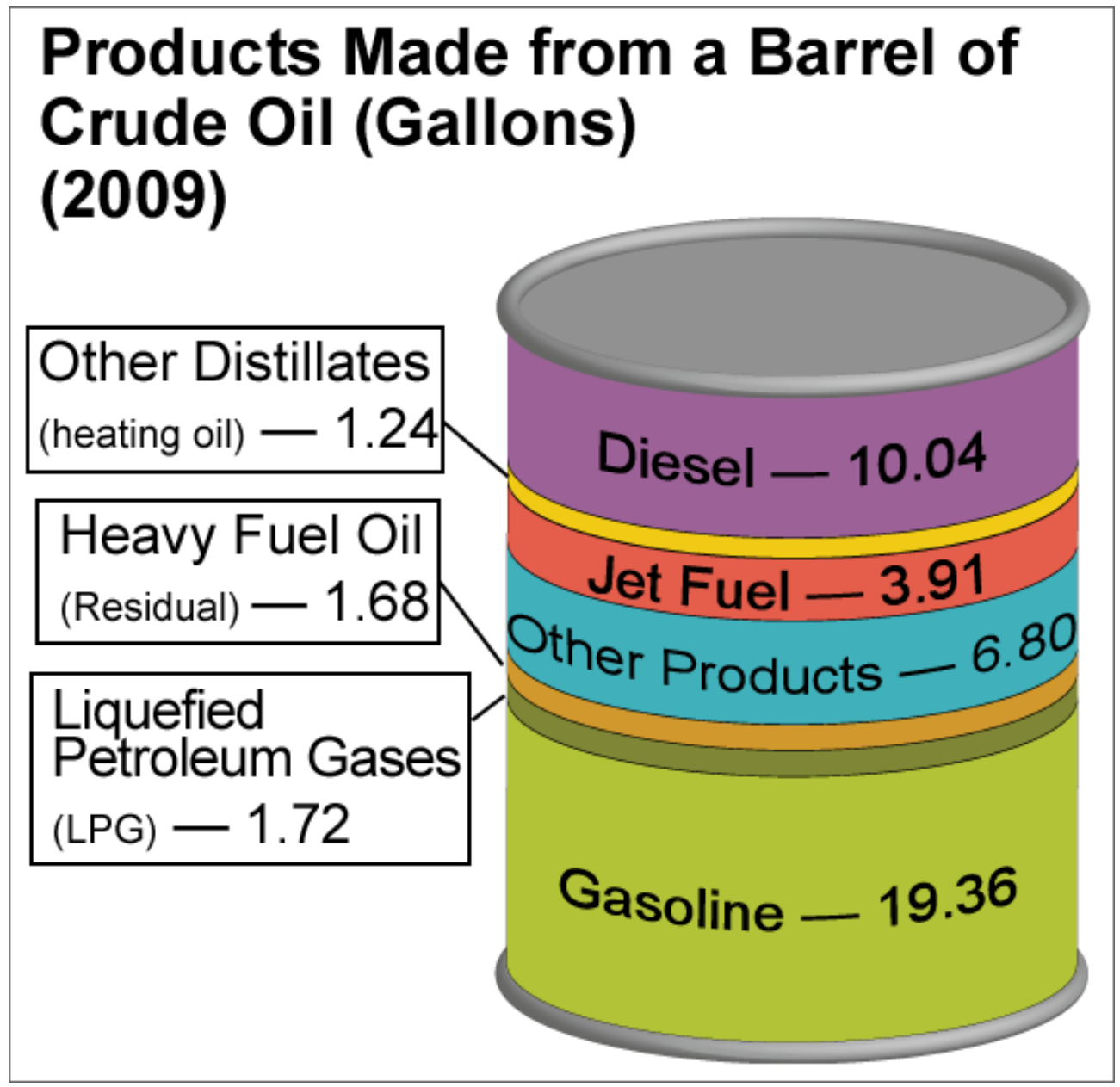

Note: Product yields are based on 2009 U.S. refining data. 


\section{Supporting Information}

\begin{tabular}{|l|c|c|}
\hline \multicolumn{3}{|c|}{ Products Made from a Barrel of Crude Oil } \\
\hline \multicolumn{1}{|c|}{ Products } & Gallons & Percent \\
\hline Gasoline & 19.36 & $43.3 \%$ \\
\hline Diesel & 10.04 & $22.4 \%$ \\
\hline Jet Fuel & 3.91 & $8.7 \%$ \\
\hline Liquefied Petroleum & 1.72 & $3.8 \%$ \\
\hline Gases & 1.68 & $3.8 \%$ \\
\hline Heavy Fuel Oil & 1.24 & $2.8 \%$ \\
\hline Other Distillates & 6.80 & $15.2 \%$ \\
\hline Other Products & 44.75 & $100.0 \%$ \\
\hline Total & $\begin{array}{l}\text { Tource } \\
\text { What Fuels Are Made from Crude Oil? }\end{array}$ \\
\hline
\end{tabular}




\section{u.s. oppapinuervof of Energy Efficiency \& \\ Renewable Energy}

\section{Vehicle Technologies Program}

Fact \#677: May 30, 2011

Number of Hybrid Models, 2001-2011

In 2001 there were only 2 hybrid models for consumers to choose from. Ten years later, the number of hybrid models available to consumers has increased to 29 models.

Between the 2010 and 2011 model year, the number of hybrids increased by 9 models, the greatest increase yet.

Number of Hybrid Models, 2001-2011

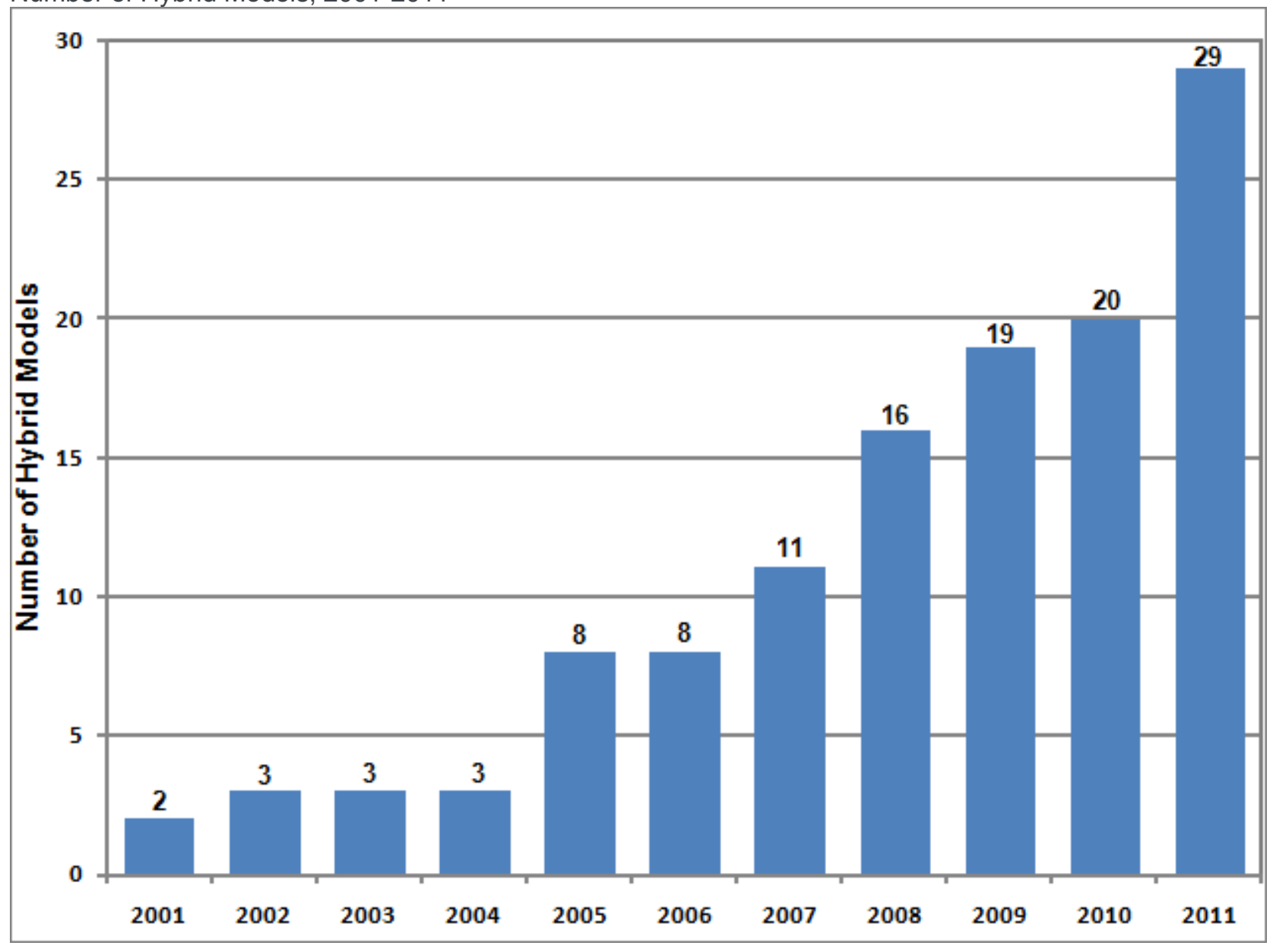




\section{Supporting Information}

\begin{tabular}{|c|c|}
\hline \multicolumn{2}{|c|}{$\begin{array}{c}\text { Number of Hybrid Models Available, 2001-2011 } \\
\text { Year }\end{array}$} \\
\hline 2001 & 2 \\
\hline 2002 & 3 \\
\hline 2003 & 3 \\
\hline 2004 & 3 \\
\hline 2005 & 8 \\
\hline 2006 & 8 \\
\hline 2007 & 11 \\
\hline 2008 & 16 \\
\hline 2009 & 19 \\
\hline 2010 & 20 \\
\hline 2011 & 29 \\
\hline $\begin{array}{l}\text { Source: U.S. Department of Energy, Alternative Fuels } \\
\text { and Advanced Vehicles Data Center, OEM } \\
\text { AFV/HEV/Diesel Light Duty Model Offerings by Fuel Type }\end{array}$ \\
\hline \begin{tabular}{l} 
1991-2011. \\
\hline
\end{tabular}
\end{tabular}




\section{u.s. Depaatment of | Energy Efficiency \& \\ Renewable Energy}

\section{Vehicle Technologies Program}

Fact \#678: June 6, 2011

Manufacturer Market Share of Hybrid Vehicles, 2010

From a total of 274,210 hybrid vehicle sales in 2010 , over two thirds (69\%) were manufactured by the Toyota Motor Company. Ford and Honda together accounted for about a quarter of hybrid vehicle sales while GM and Nissan together sold about 5\%. Other manufacturers including Porsche, Mazda, Mercedes and BMW totaled less than $1 \%$ of hybrid vehicle sales.

Manufacturer Market Share of Hybrid Vehicles, 2010

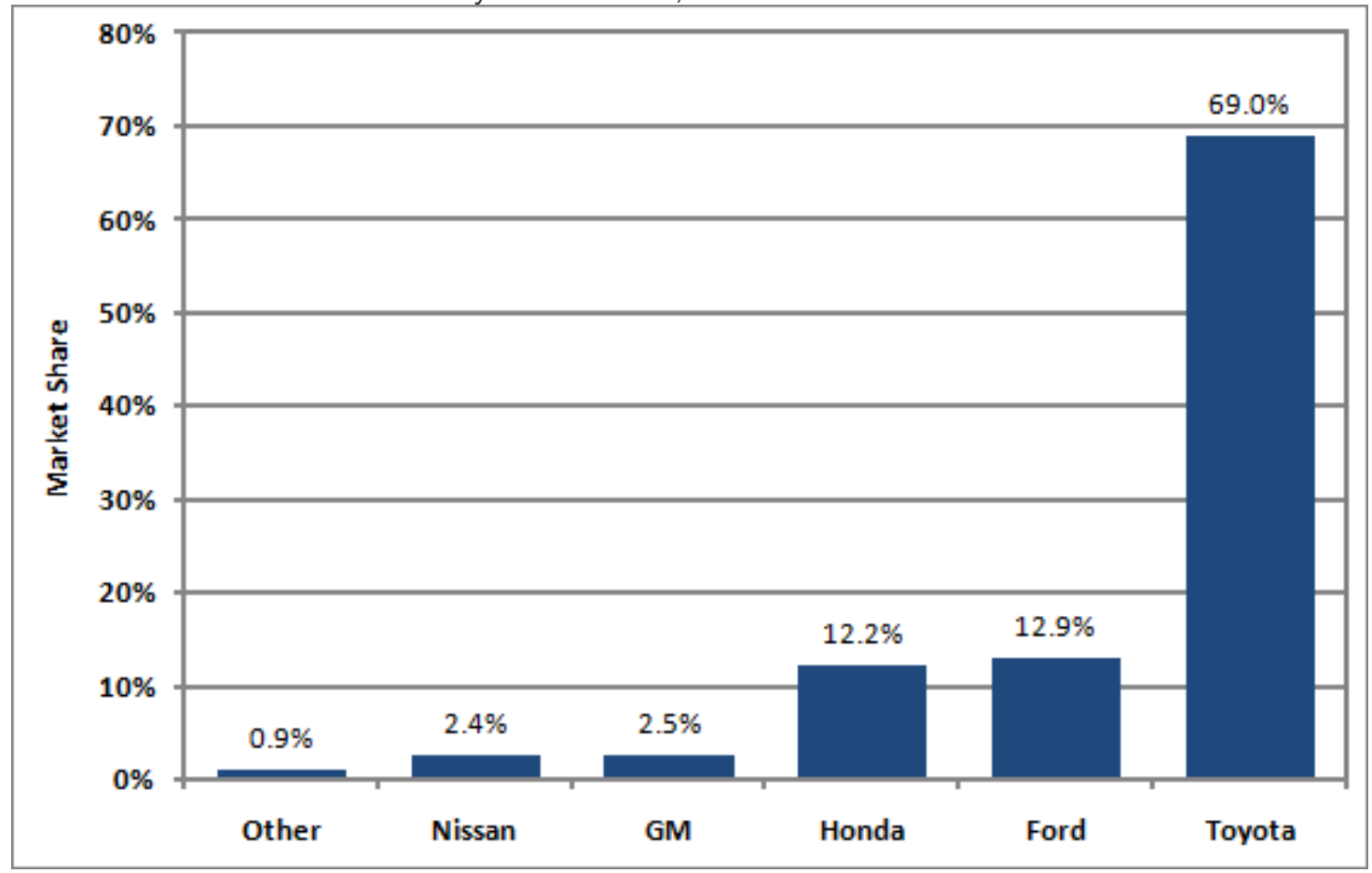

Note: Other includes Porsche, Mazda, Mercedes and BMW. 


\section{Supporting Information}

\begin{tabular}{|c|c|c|}
\hline \multicolumn{3}{|c|}{ Manufacturer Market Share of Hybrid Vehicles, 2010} \\
\hline Manufacturer & Sales & Market Share \\
\hline Toyota & 189,187 & $69.0 \%$ \\
\hline Ford & 35,496 & $12.9 \%$ \\
\hline Honda & 33,547 & $12.2 \%$ \\
\hline GM & 6,759 & $2.5 \%$ \\
\hline Nissan & 6,710 & $2.4 \%$ \\
\hline Other & 2,511 & $0.9 \%$ \\
\hline Total & 274,210 & $100.0 \%$ \\
\hline \multicolumn{3}{|c|}{$\begin{array}{l}\text { Source: U.S. Department of Energy, Alternative Fuels \& } \\
\text { Advanced Vehicles Data Center, HEV Sales by Model. }\end{array}$} \\
\hline
\end{tabular}




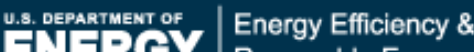 \\ Renewable Energy}

\section{Vehicle Technologies Program}

\section{Fact \#679: June 13, 2011}

\section{U.S. Imports of Fuel Ethanol Drop Sharply}

U.S. imports of fuel ethanol were low until 2004 when imports began to rise sharply. By 2006 imports of fuel ethanol reached a record high of 735.8 million gallons. As domestic supply of fuel ethanol increased to meet demand, imports of fuel ethanol began to drop after 2006. By 2010, imports declined to pre-2004 levels.

Annual U.S. Imports of Fuel Ethanol, 2001-2010

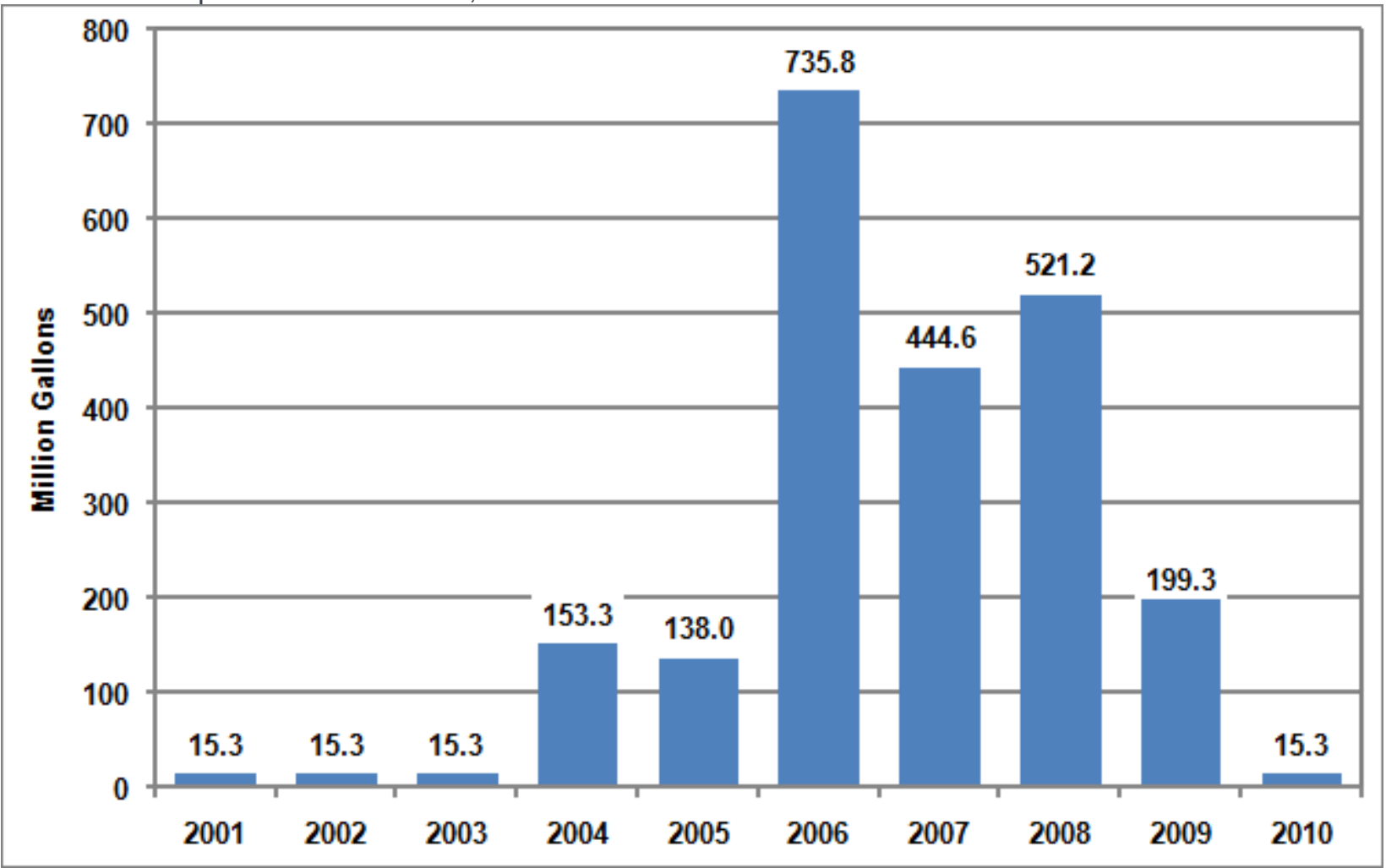




\section{Supporting Information}

\begin{tabular}{|c|c|}
\hline \multicolumn{2}{|c|}{$\begin{array}{c}\text { U.S. Imports of Fuel Ethanol, 2001-2010 } \\
\text { Year }\end{array}$} \\
\hline 2001 & 15.3 \\
\hline 2002 & 15.3 \\
\hline 2003 & 15.3 \\
\hline 2004 & 153.3 \\
\hline 2005 & 138.0 \\
\hline 2006 & 735.8 \\
\hline 2007 & 444.6 \\
\hline 2008 & 521.2 \\
\hline 2009 & 199.3 \\
\hline 2010 & 15.3 \\
\hline Source: Energy Information Administration, \\
Annual Imports of U.S. Fuel Ethanol.
\end{tabular}




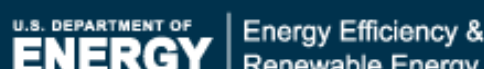 \\ Renewable Energy \\ Vehicle Technologies Program}

Fact \#680: June 20, 2011

Fuel Economy is "Most Important" When Buying a Vehicle

A June 2011 survey asked the question "Which one of the following attributes would be MOST important to you in your choice of your next vehicle?" The choices were fuel economy, dependability, low price, quality, and safety. This same question was asked in previous surveys and the results are compared in the graph below. Dependability was chosen most often in nearly every survey, but fuel economy surpassed it in 2011 . Thirty percent of the survey respondents indicated that fuel economy would be the most important vehicle attribute, while only $22 \%$ of respondents chose dependability.

Most Important Vehicle Attribute

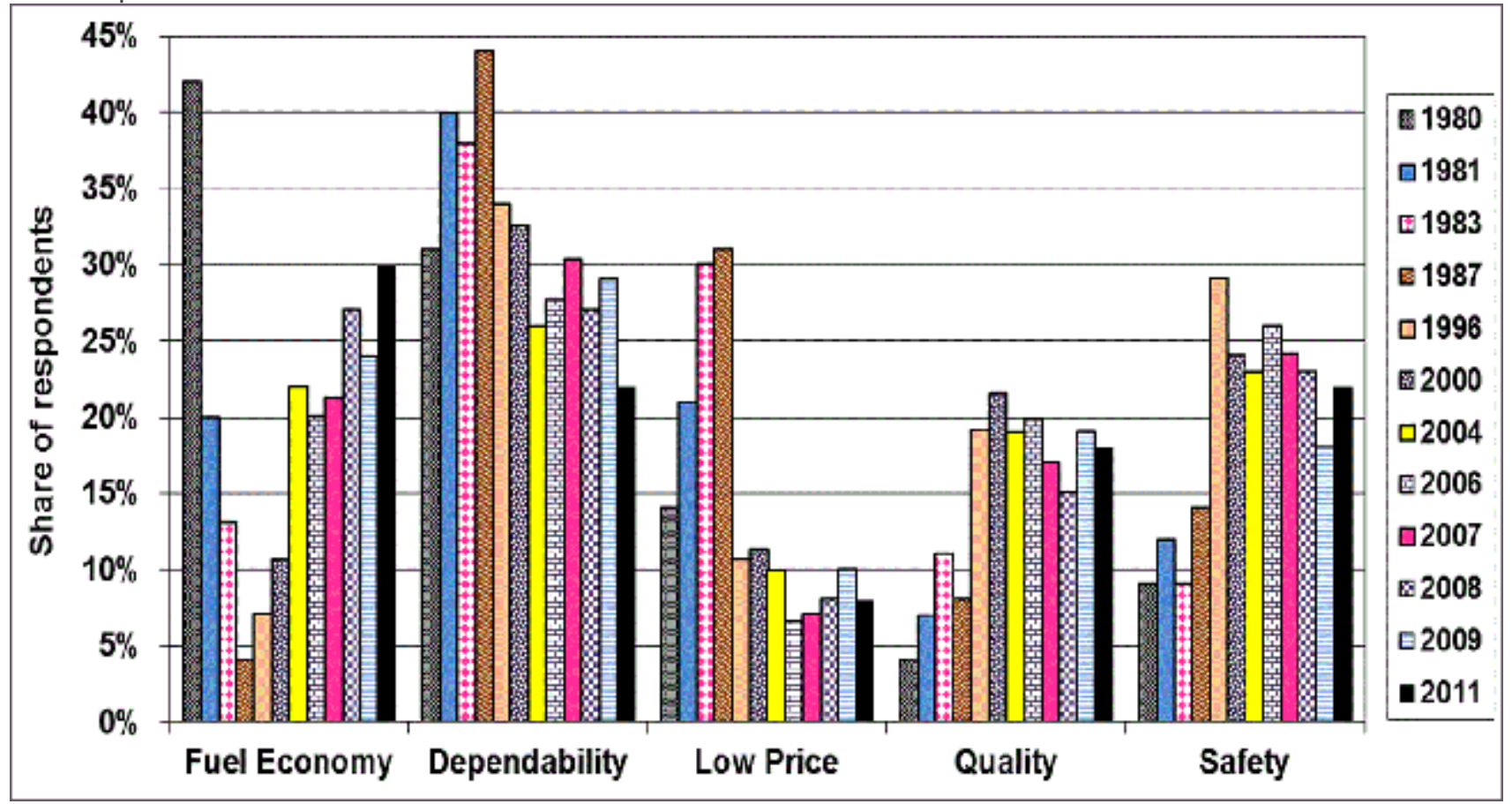




\section{Supporting Information}

\begin{tabular}{|c|c|c|c|c|c|c|}
\hline \multicolumn{7}{|c|}{$\begin{array}{c}\text { Q: Which one of the following attributes would be most important in your choice of your next } \\
\text { vehicle? }\end{array}$} \\
\hline Year & Fuel Economy & Dependability & Low Price & Quality & Safety & Total \\
\hline 1980 & $42 \%$ & $31 \%$ & $14 \%$ & $4 \%$ & $9 \%$ & $100 \%$ \\
\hline 1981 & $20 \%$ & $40 \%$ & $21 \%$ & $7 \%$ & $12 \%$ & $100 \%$ \\
\hline 1983 & $13 \%$ & $38 \%$ & $30 \%$ & $11 \%$ & $9 \%$ & $101 \%$ \\
\hline 1985 & $8 \%$ & $41 \%$ & $29 \%$ & $12 \%$ & $10 \%$ & $100 \%$ \\
\hline 1987 & $4 \%$ & $44 \%$ & $31 \%$ & $8 \%$ & $14 \%$ & $101 \%$ \\
\hline 1996 & $7 \%$ & $34 \%$ & $11 \%$ & $19 \%$ & $29 \%$ & $100 \%$ \\
\hline 2000 & $11 \%$ & $33 \%$ & $11 \%$ & $22 \%$ & $24 \%$ & $100 \%$ \\
\hline 2001 & $11 \%$ & $30 \%$ & $8 \%$ & $22 \%$ & $30 \%$ & $100 \%$ \\
\hline 2004 & $22 \%$ & $26 \%$ & $10 \%$ & $19 \%$ & $23 \%$ & $100 \%$ \\
\hline 2005 & $12 \%$ & $33 \%$ & $7 \%$ & $21 \%$ & $28 \%$ & $100 \%$ \\
\hline 2006 & $20 \%$ & $28 \%$ & $7 \%$ & $20 \%$ & $26 \%$ & $100 \%$ \\
\hline 2007 & $21 \%$ & $30 \%$ & $7 \%$ & $17 \%$ & $24 \%$ & $100 \%$ \\
\hline 2008 & $27 \%$ & $27 \%$ & $8 \%$ & $15 \%$ & $23 \%$ & $100 \%$ \\
\hline 2009 & $24 \%$ & $29 \%$ & $10 \%$ & $19 \%$ & $18 \%$ & $100 \%$ \\
\hline 2011 & $30 \%$ & $22 \%$ & $8 \%$ & $18 \%$ & $22 \%$ & $100 \%$ \\
\hline \multicolumn{7}{|c|}{ 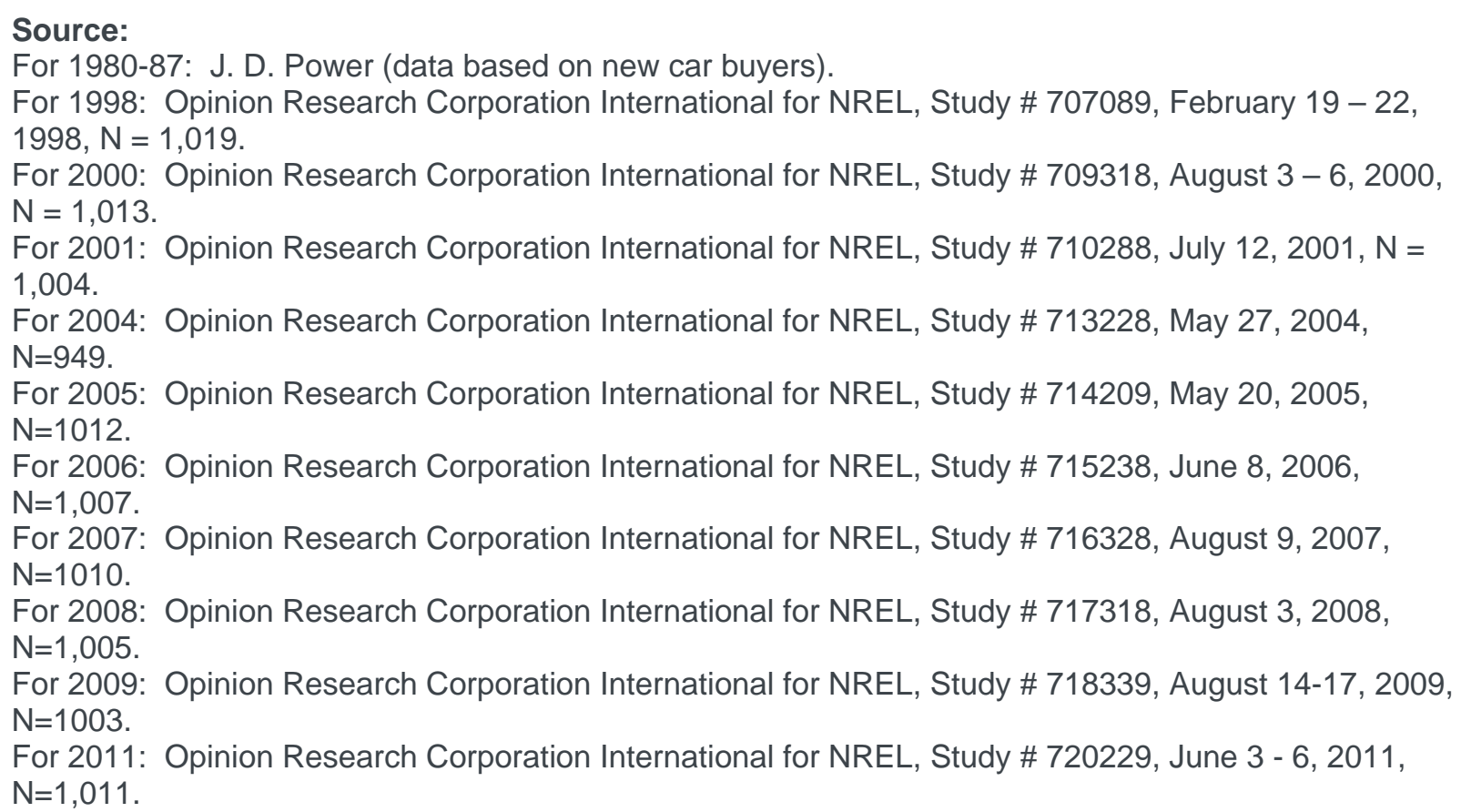 } \\
\hline
\end{tabular}




\section{Vehicle Technologies Program}

\section{Fact \#681: June 27, 2011}

\section{U.S. Ethanol Production, 2001-2010}

Ethanol production has been rising rapidly over the last 10 years driven largely by government standards requiring the use of renewable fuels. The first Renewable Fuels Standard (RFS) was established in 2005 and required the use of 4 billion gallons of renewable fuels by 2006 and 7.5 billion gallons by 2012. In 2007, the Energy Independence and Security Act superseded and expanded the original RFS to require an annual use of 9 billion gallons of biofuels in 2008 and 36 billion gallons to be used annually by 2022. Because ethanol is the dominant biofuel in the U.S., there is a strong relationship between the RFS standards and ethanol production.

U.S. Fuel Ethanol Production, 2010

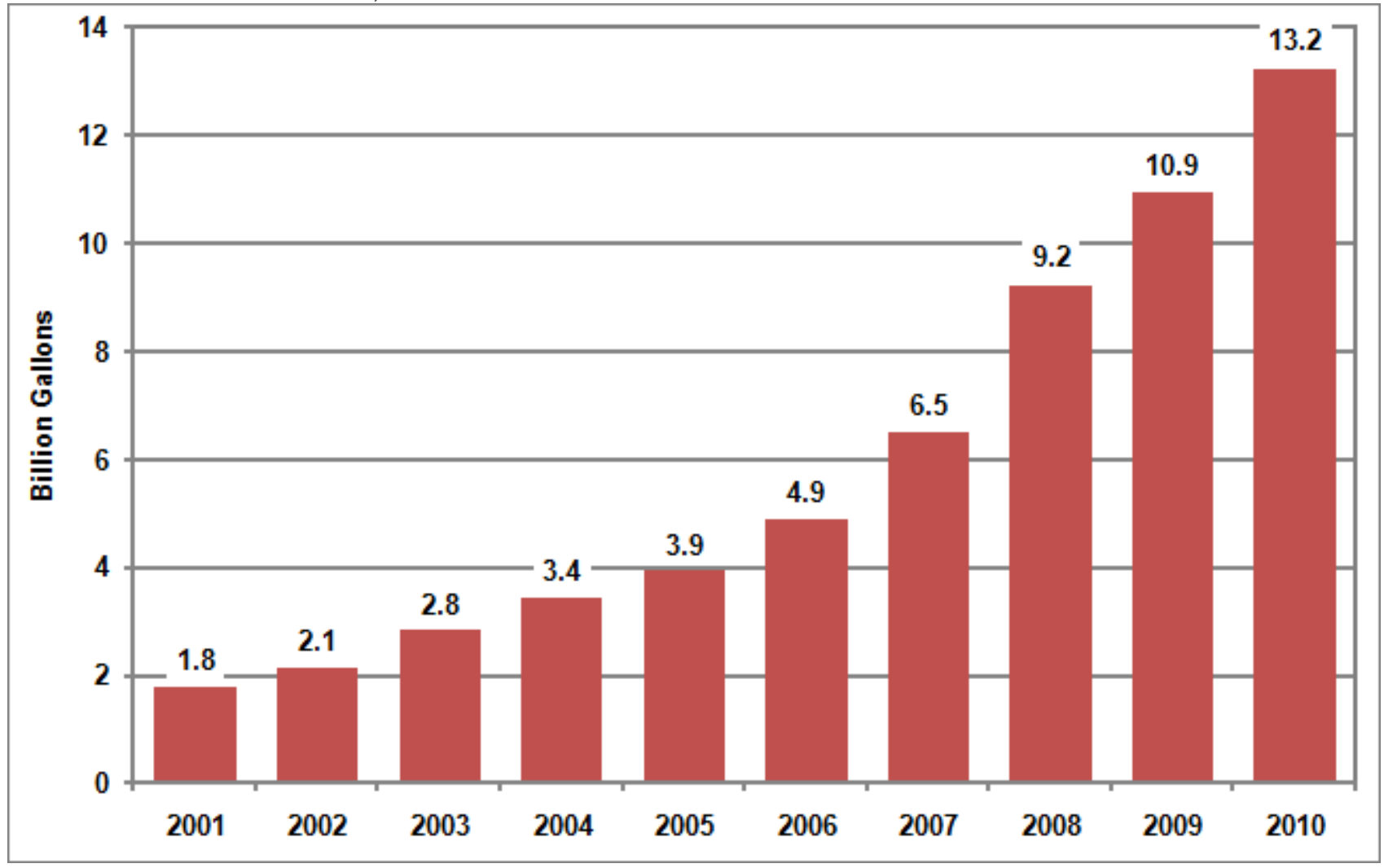




\section{Supporting Information}

\begin{tabular}{|c|c|}
\hline \multicolumn{2}{|c|}{ U.S. Fuel Ethanol Production } \\
\hline Year & Billion Gallons \\
\hline 2001 & 1.8 \\
\hline 2002 & 2.1 \\
\hline 2003 & 2.8 \\
\hline 2004 & 3.4 \\
\hline 2005 & 3.9 \\
\hline 2006 & 4.9 \\
\hline 2007 & 6.5 \\
\hline 2008 & 9.2 \\
\hline 2009 & 10.9 \\
\hline 2010 & 13.2 \\
\hline Sources: & \\
\hline 2001 - 2008: Energy Information Administration, \\
\hline EIA-819 Monthly Oxygenate Report Historical. \\
\hline 2009 - 2010: Energy Information Administration, \\
\hline April Petroleum Supply Monthly, Table 1. \\
\hline
\end{tabular}




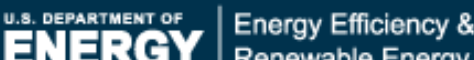 \\ Renewable Energy}

\section{Vehicle Technologies Program}

\section{Fact \#682: July 4, 2011}

\section{Federal Alternative Fuel Use}

The Federal Government used nearly 9 million gasoline-gallon equivalents of alternative fuel in 2010. The majority of the fuel used (92\%) was E-85, a combination of $85 \%$ ethanol and 15\% gasoline. The Government's use of liquefied petroleum gas (LPG), compressed natural gas (CNG), and liquefied natural gas (LNG) has declined over the last few years. In 2010, electricity use grew due to a large acquisition of electric vehicles for the Federal fleet.

Federal Alternative Fuel Use, 2006-2010

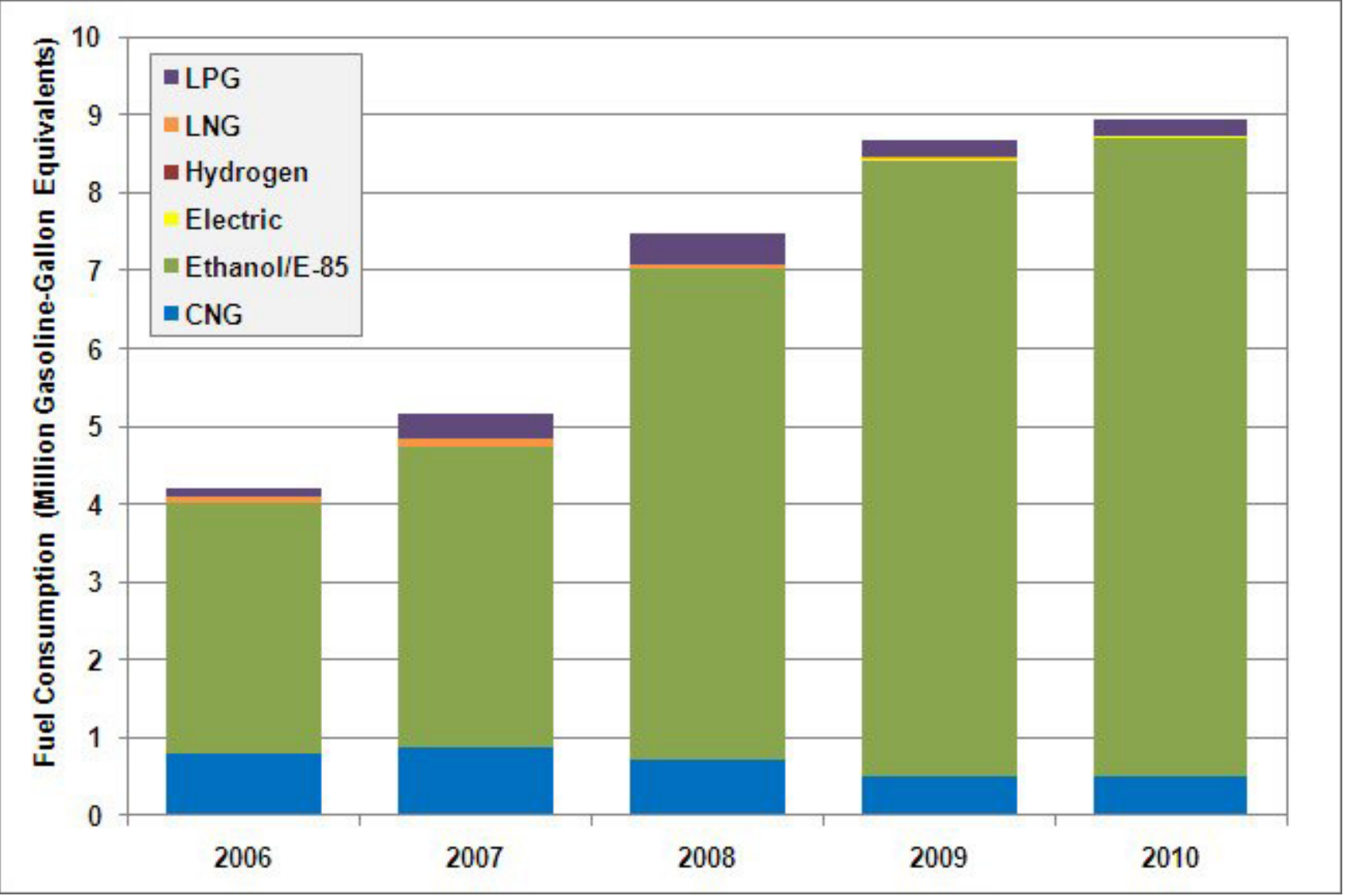




\section{Supporting Information}

\begin{tabular}{|l|r|r|r|r|r|}
\hline \multicolumn{7}{|c|}{ Federal Alternative Fuel Use (Gasoline-gallon Equivalents) } \\
\hline Fuel Type & \multicolumn{1}{|c|}{$\mathbf{2 0 0 6}$} & $\mathbf{2 0 0 7}$ & $\mathbf{2 0 0 8}$ & $\mathbf{2 0 0 9}$ & $\mathbf{2 0 1 0}$ \\
\hline CNG & 806,985 & 888,694 & 731,228 & 499,249 & 503,535 \\
\hline Electric & 5,449 & 5,316 & 4,357 & 3,996 & 36,383 \\
\hline Ethanol/E-85 & $3,205,693$ & $3,853,449$ & $6,292,555$ & $7,921,649$ & $8,200,471$ \\
\hline LNG & 89,577 & 94,512 & 59,302 & 34,939 & 481 \\
\hline LPG & 105,135 & 321,545 & 399,069 & 207,564 & 194,868 \\
\hline Hydrogen & 0 & 0 & 38 & 431 & 790 \\
\hline Total & $4,212,839$ & $5,163,516$ & $7,486,549$ & $8,667,828$ & $8,936,528$ \\
\hline $\begin{array}{l}\text { Source: } \\
\text { General Services }\end{array}$ & Administration, 2010 Federal Fleet Report, Table 5-1. \\
\hline
\end{tabular}




\section{Vehicle Technologies Program}

\section{Fact \#683: July 11, 2011 \\ Federal Tax Credits for the Purchase of Advanced Technology Vehicles}

The Federal Government has encouraged the use of different transportation fuels by allowing tax credits on vehicle purchases. The purchase of a traditional (non-plug-in) hybrid vehicle was eligible for a tax credit of up to \$3,400 from 2005 through 2010 . Diesels, which are more efficient than gasoline vehicles, were eligible for a similar tax credit, as were alternative-fuel vehicles. All of those credits were discontinued at the end of calendar year 2010. Now, electric vehicles and plug-in hybrid-electric vehicles are the only ones for which a tax credit is available - up to $\$ 7,500$. To find out more about these tax credits, go to the IRS website, or FuelEconomy.Gov.

\begin{tabular}{|c|c|c|c|}
\hline Federal & jovernment Tax I & entives for & vanced Technology Vehicles \\
\hline Vehicle Type & $\begin{array}{l}\text { Calendar Year } \\
\text { in which } \\
\text { the Vehicle was } \\
\text { Purchased }\end{array}$ & $\begin{array}{c}\text { Maximum } \\
\text { Credit } \\
\text { Amount }\end{array}$ & $\begin{array}{c}\text { Vehicles Currently Eligible for a Tax } \\
\text { Credit }\end{array}$ \\
\hline $\begin{array}{l}\text { Plug-in Hybrid- } \\
\text { Electric Vehicles }\end{array}$ & 2010 - on* & $\$ 7,500$ & $\begin{array}{l}2011 \\
\text { Chevrolet Volt }\end{array}$ \\
\hline \multirow{3}{*}{ Electric Vehicles } & \multirow{3}{*}{$2009-$ on*\# $^{*}$} & \multirow{3}{*}{$\$ 7,500$} & $\begin{array}{l}2010 \text { Coda } \\
\text { Sedan }\end{array}$ \\
\hline & & & $\begin{array}{l}2011 \text { Nissan } \\
\text { Leaf }\end{array}$ \\
\hline & & & $\begin{array}{l}2011 \text { Smart } \\
\text { For Two }\end{array}$ \\
\hline
\end{tabular}




\begin{tabular}{|l|l|l|}
\hline & \\
\hline
\end{tabular}




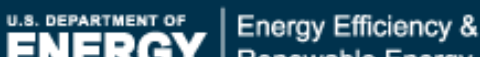 \\ Renewable Energy}

\section{Vehicle Technologies Program}

Fact \#684: July 18, 2011

Fuel Economy versus Fuel Savings

An increase in fuel economy by 5 miles per gallon $(\mathrm{mpg})$ does not translate to a constant fuel savings amount. Thus, trading a low-mpg car or truck for one with just slightly better mpg will save more fuel than trading a high-mpg car or truck for one that is even higher. For example, trading a truck that gets $15 \mathrm{mpg}$ for a new one that gets 20 mpg will save 16.7 gallons of fuel for every 1,000 miles driven. In contrast, trading a 35 $\mathrm{mpg}$ car for a new car that gets $40 \mathrm{mpg}$ will save 3.6 gallons of fuel for every 1,000 miles driven. These fuel savings are additive; that is, going from $15 \mathrm{mpg}$ to $25 \mathrm{mpg}$ saves 26.7 gallons per thousand miles driven -16.7 gallons (15-20 mpg difference) plus 10.0 gallons (20-25 mpg difference).

Fuel Savings per Thousand Miles

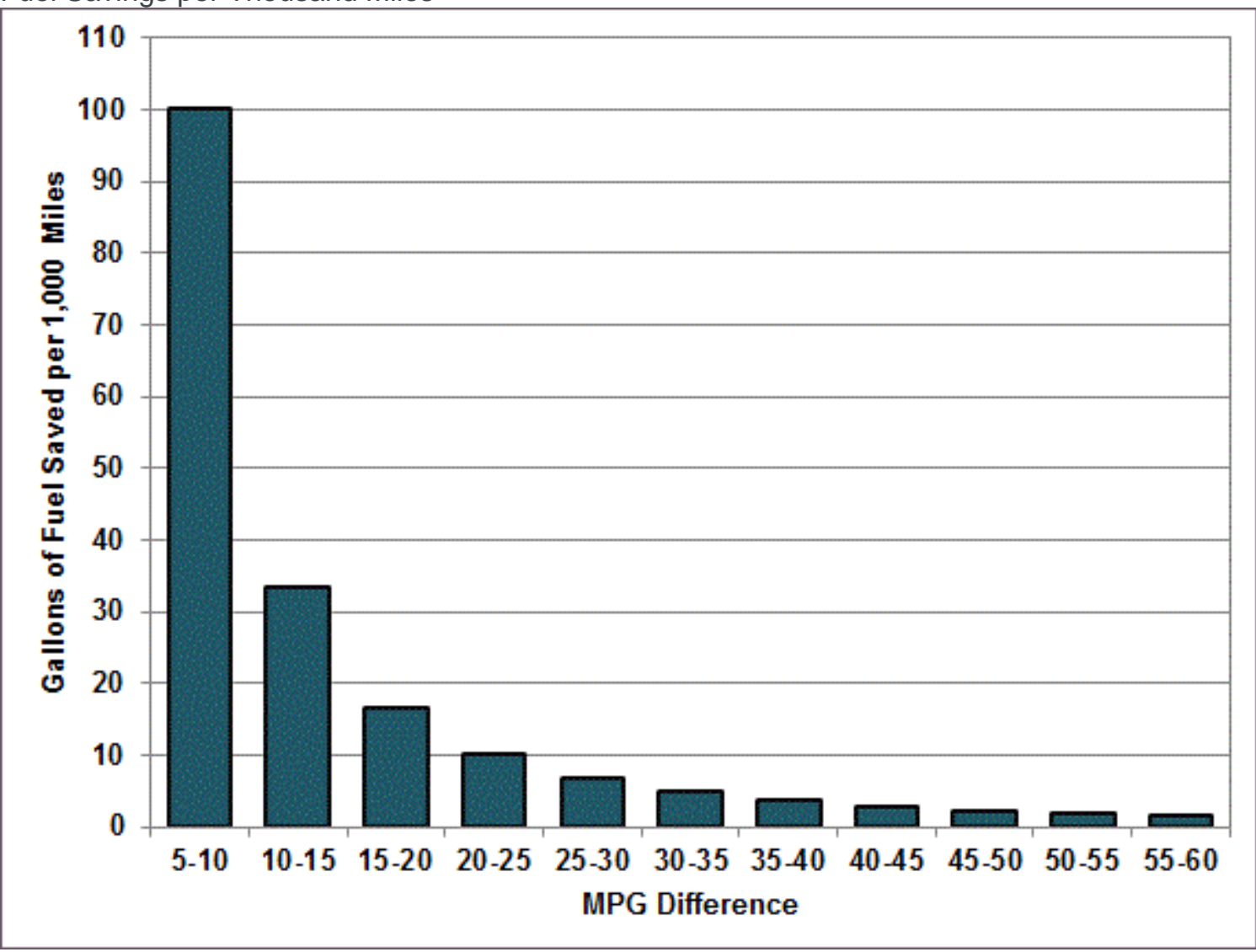




\section{Supporting Information}

\begin{tabular}{|c|c|c|}
\hline $\begin{array}{c}\text { If you have a car } \\
\text { with this fuel } \\
\text { economy... }\end{array}$ & $\begin{array}{c}\text { And you trade it for a } \\
\text { car with this fuel } \\
\text { economy... }\end{array}$ & $\begin{array}{c}\text { You save this many } \\
\text { gallons for every 1,000 } \\
\text { miles you drive }\end{array}$ \\
\hline $5 \mathrm{mpg}$ & $10 \mathrm{mpg}$ & 100.00 \\
\hline $10 \mathrm{mpg}$ & $15 \mathrm{mpg}$ & 33.3 \\
\hline $15 \mathrm{mpg}$ & $20 \mathrm{mpg}$ & 16.7 \\
\hline $20 \mathrm{mpg}$ & $25 \mathrm{mpg}$ & 10.0 \\
\hline $25 \mathrm{mpg}$ & $30 \mathrm{mpg}$ & 6.7 \\
\hline $30 \mathrm{mpg}$ & $35 \mathrm{mpg}$ & 4.8 \\
\hline $35 \mathrm{mpg}$ & $40 \mathrm{mpg}$ & 3.6 \\
\hline $40 \mathrm{mpg}$ & $45 \mathrm{mpg}$ & 2.8 \\
\hline $45 \mathrm{mpg}$ & $50 \mathrm{mpg}$ & 2.2 \\
\hline $50 \mathrm{mpg}$ & $55 \mathrm{mpg}$ & 1.8 \\
\hline $55 \mathrm{mpg}$ & $60 \mathrm{mpg}$ & 1.5 \\
\hline
\end{tabular}

Source:

U.S. Department of Energy and Environmental Protection Agency, Fuel Economy Guide Website. 


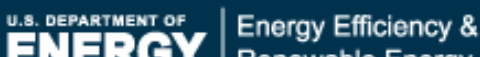 \\ Renewable Energy}

\section{Vehicle Technologies Program}

Fact \#685: July 25, 2011

Reasons for Buying a Plug-in Hybrid Vehicle

General Motors has been gathering feedback from customers who purchased the 2011 Chevrolet Volt, which is the only plug-in hybrid vehicle (PHEV) on the market today. Through May 2011, about 2,100 Volts have been sold. The top reason cited for purchasing a Volt is to reduce America's dependence on foreign oil. A desire to buy American and the vehicle's extended range by using gasoline tied for second. Environmental concerns were fifth on the list of reasons to purchase the vehicle. According to General Motors, current Volt owners drive two-thirds of the time in electric mode, and travel an average of 900 miles before refilling the gasoline tank.

Reasons for Buying a PHEV

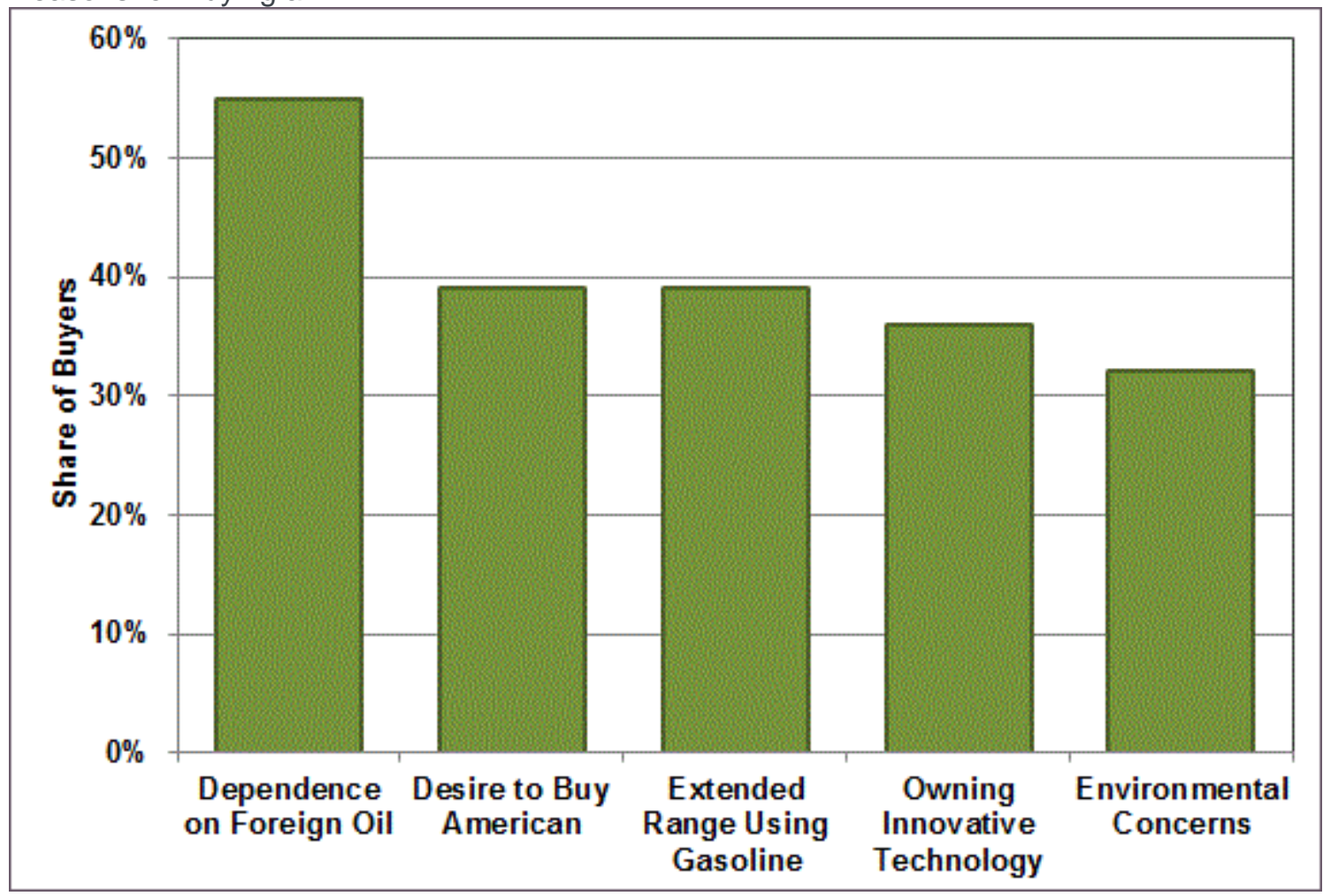




\section{Supporting Information}

\begin{tabular}{|c|c|}
\hline Reasons for Buying a PHEV & Share of Buyers \\
\hline Dependence on Foreign Oil & $55 \%$ \\
\hline Desire to Buy American & $39 \%$ \\
\hline Extended Range Using Gasoline & $39 \%$ \\
\hline Owning Innovative Technology & $36 \% *$ \\
\hline Environmental Concerns & $32 \%$ \\
\hline \multicolumn{2}{|c|}{$\begin{array}{l}\text { * Estimated. Exact percentage cannot be determined from } \\
\text { available information. However, the actual number is between } \\
32 \% \text { and } 39 \% \text {. } \\
\text { Sources: Crain Communications, Automotive News, June 20, } \\
\text { 2011. [Original source: General Motors.] Cristi Landy, } \\
\text { Chevrolet Volt Marketing Director }\end{array}$} \\
\hline
\end{tabular}




\section{Vehicle Technologies Program}

\section{Fact \#686: August 1, 2011 Emissions and Energy Use Model - GREET}

The Greenhouse Gases, Regulated Emission, and Energy Use in Transportation (GREET) Model is a full life-cycle model for evaluating the energy and emission impacts of various vehicle and fuel combinations. The first version of the GREET model was released in 1996. Since then, the model has been updated and expanded to include additional vehicle types and fuels. The results below are from GREET Model 1.8d.1. The California grid mix (bars outlined in green) was chosen due to the high renewable energy mix in that state. In contrast, the West Virginia grid mix (bars outlined in black) is primarily coal. Both of these are compared against the average U.S. grid mix for various vehicle technologies.

Well-to-Wheel Emissions for Various Fuels and Vehicle Technologies

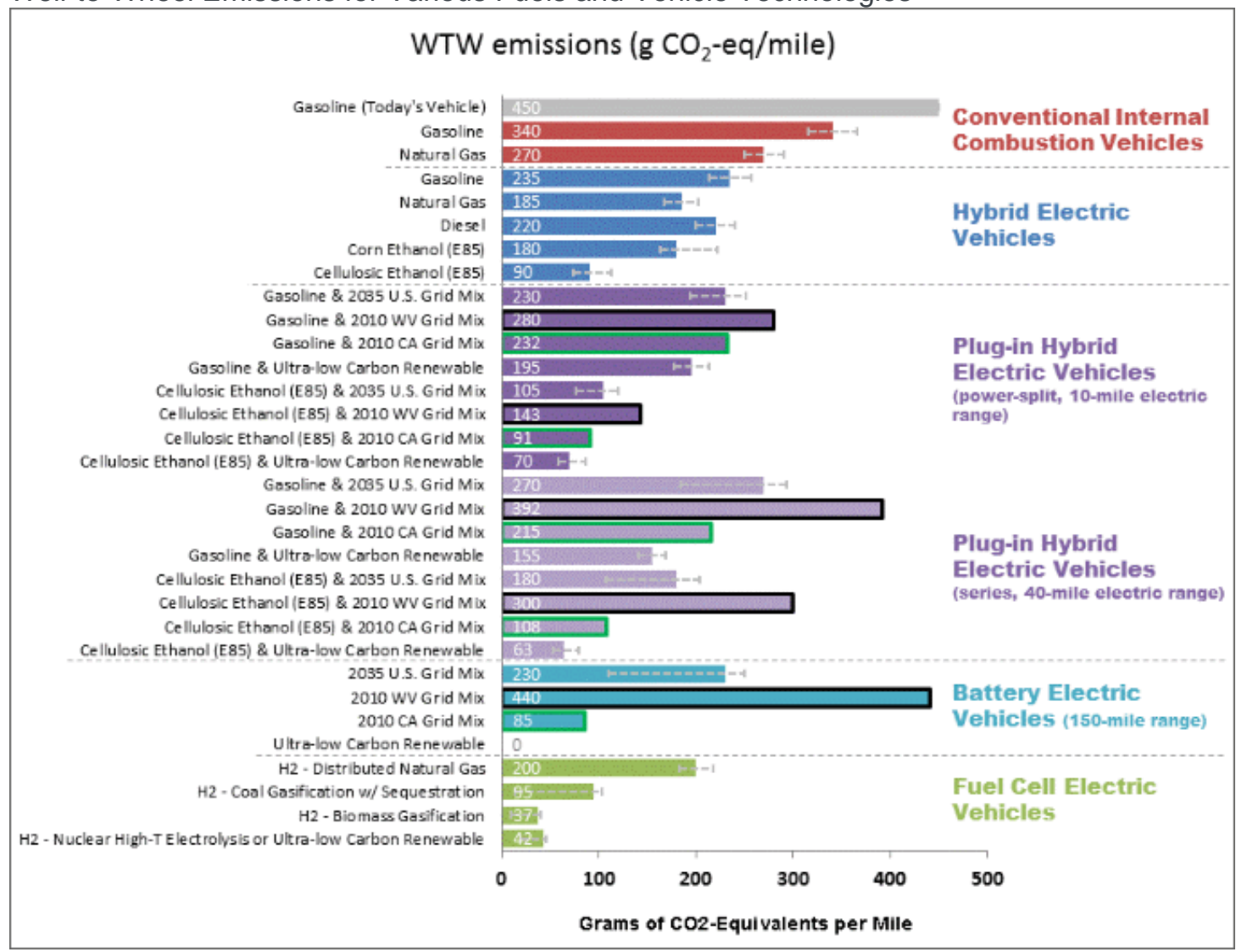

Note: $\mathrm{H} 2$ = hydrogen; High-T = high-temperature 


\section{Supporting Information}

\begin{tabular}{|c|c|c|}
\hline \multicolumn{3}{|c|}{ Well-to-Wheel Emissions for Various Fuels and Vehicle Technologies } \\
\hline Fuel & $\begin{array}{l}\text { Grams of CO2- } \\
\text { Equivalent } \\
\text { per Mile }\end{array}$ & Vehicle Technology \\
\hline Gasoline (Today's Vehicle) & 450 & \multirow{3}{*}{$\begin{array}{l}\text { Conventional Internal } \\
\text { Combustion Vehicles }\end{array}$} \\
\hline Gasoline & 340 & \\
\hline Natural Gas & 270 & \\
\hline Gasoline & 235 & \multirow{5}{*}{$\begin{array}{l}\text { Hybrid Electric } \\
\text { Vehicles }\end{array}$} \\
\hline Natural Gas & 185 & \\
\hline Diesel & 220 & \\
\hline Corn Ethanol (E85) & 180 & \\
\hline Cellulosic Ethanol (E85) & 90 & \\
\hline Gasoline \& 2035 U.S. Grid Mix & 230 & \multirow{8}{*}{$\begin{array}{l}\text { Plug-in Hybrid Electric } \\
\text { Vehicles } \\
\text { (power-split, 10-mile } \\
\text { electric range) }\end{array}$} \\
\hline Gasoline \& 2010 WV Grid Mix & 280 & \\
\hline Gasoline 2010 CA Grid Mix & 232 & \\
\hline $\begin{array}{r}\text { Gasoline \& Ultra-low Carbon } \\
\text { Renewable }\end{array}$ & 195 & \\
\hline $\begin{array}{r}\text { Cellulosic Ethanol (E85) \& } \\
2035 \text { U.S. Grid Mix }\end{array}$ & 105 & \\
\hline $\begin{array}{r}\text { Cellulosic Ethanol (E85) \& } \\
2010 \text { WV Grid Mix }\end{array}$ & 143 & \\
\hline $\begin{array}{r}\text { Cellulosic Ethanol (E85) \& } \\
2010 \text { CA Grid Mix }\end{array}$ & 91 & \\
\hline $\begin{array}{l}\text { Cellulosic Ethanol (E85) \& } \\
\text { Ultra-low carbon Renewable }\end{array}$ & 70 & \\
\hline Gasoline \& 2035 U.S. Grid Mix & 270 & \multirow{5}{*}{$\begin{array}{c}\text { Plug-in Hybrid Electric } \\
\text { Vehicles } \\
\text { (series, 40-mile } \\
\text { electric range) }\end{array}$} \\
\hline Gasoline \& 2010 WV Grid Mix & 392 & \\
\hline Gasoline \& 2010 CA Grid Mix & 215 & \\
\hline $\begin{array}{r}\text { Gasoline \& Ultra-low Carbon } \\
\text { Renewable }\end{array}$ & 155 & \\
\hline $\begin{array}{r}\text { Cellulosic Ethanol (E85) \& } \\
2035 \text { U.S. Grid Mix }\end{array}$ & 180 & \\
\hline
\end{tabular}




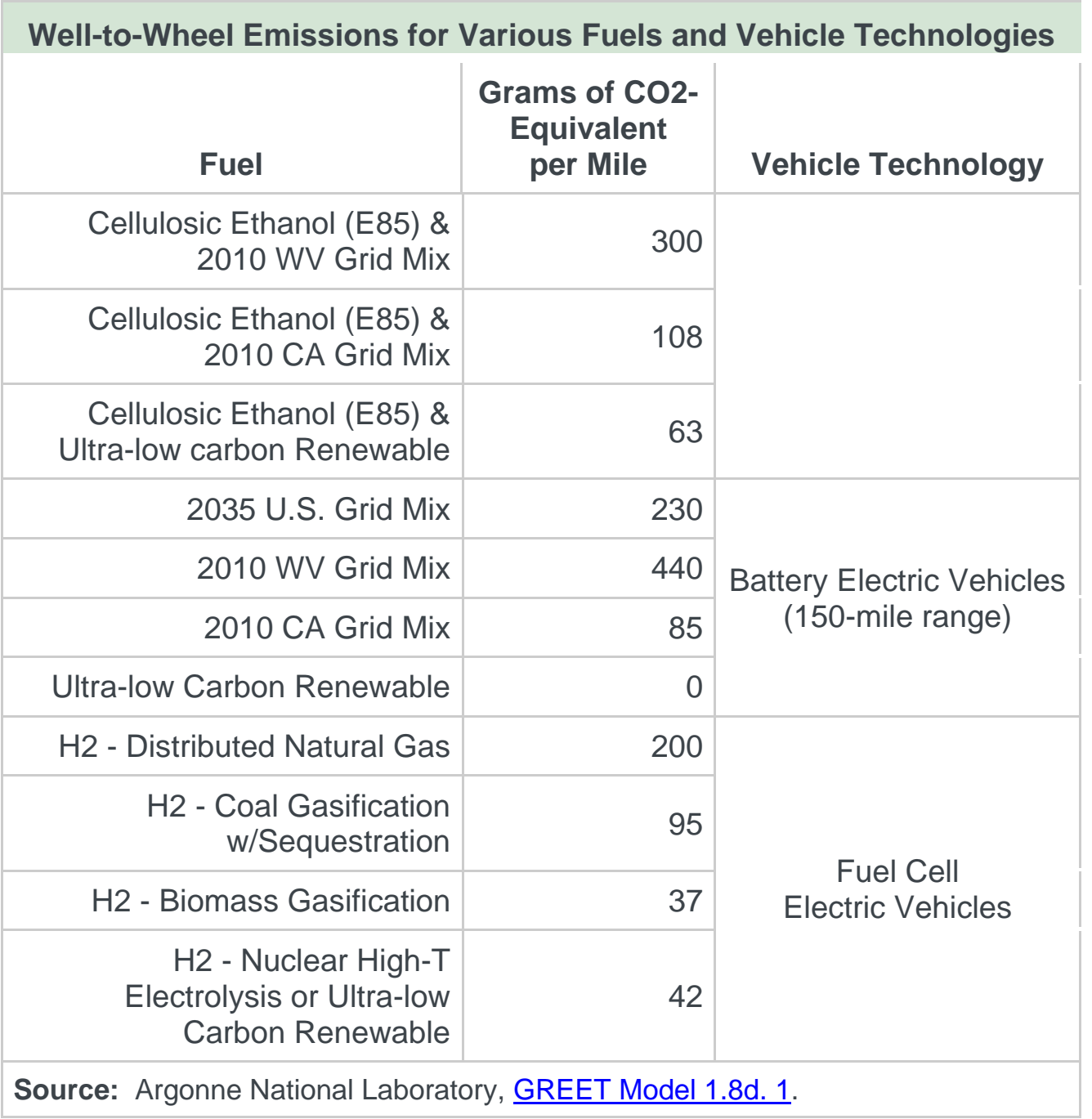




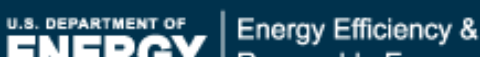 \\ Renewable Energy}

\section{Vehicle Technologies Program}

\section{Fact \#687: August 8, 2011 The Transportation Petroleum Gap}

In 1989 the transportation sector petroleum consumption surpassed U.S. petroleum production for the first time, creating a gap that must be met with imports of petroleum. By the year 2035, transportation petroleum consumption is expected to grow to almost 17 million barrels per day; at that time, the gap between U.S. production and transportation consumption will be almost 4 million barrels per day (when including the non-petroleum sources).

U.S. Petroleum Production and Transportation Consumption, 1970-2035

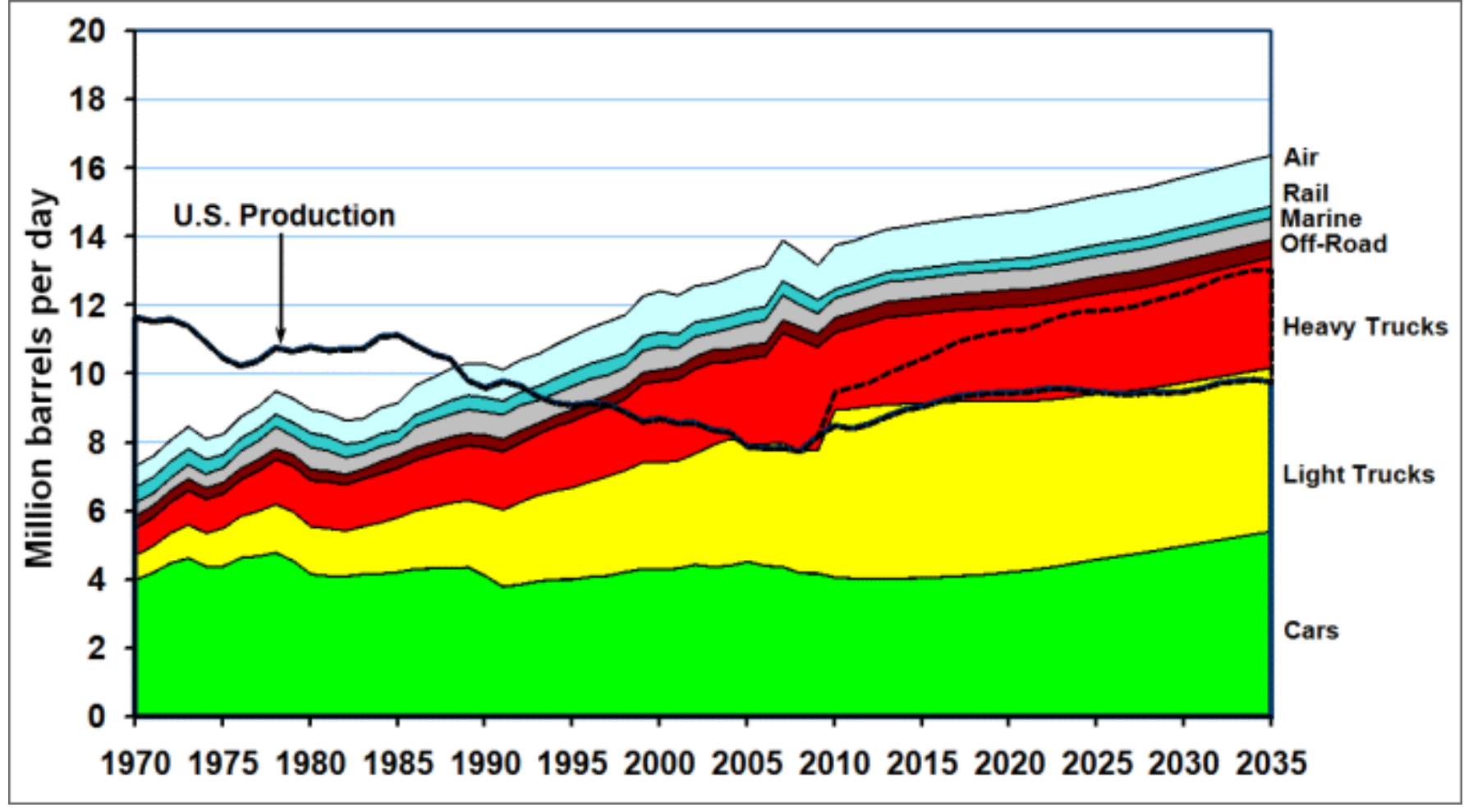

Note: The U.S. Production has two lines after 2005. The solid line is conventional sources of petroleum, including crude oil, natural gas plant liquids, and refinery gains. The dashed line adds in other nonpetroleum sources, including ethanol, biomass, liquids from coal, other blending components, other hydrocarbons, and ethers. The sharp increase in values between 2009 and 2010 is caused by the data change from historical to projected values. The sharp increase in the value for heavy trucks between 2006 and 2007 is the result of FHWA's methodology change. 


\section{Supporting Information}

\begin{tabular}{|c|c|c|c|c|c|c|c|c|c|c|c|}
\hline \multicolumn{12}{|c|}{$\begin{array}{l}\text { Historical and Future U.S. Petroleum Production and Transportation } \\
\text { Petroleum Use (Million barrels per day) }\end{array}$} \\
\hline Year & Autos & $\begin{array}{l}\text { Light } \\
\text { Trucks }\end{array}$ & $\begin{array}{c}\text { Medium } \\
\& \\
\text { Heavy } \\
\text { Trucks }\end{array}$ & Air & Water & $\begin{array}{l}\text { Off- } \\
\text { Highway }\end{array}$ & Rail & Pipeline & $\begin{array}{c}\text { Total } \\
\text { Trans- } \\
\text { portation }\end{array}$ & \begin{tabular}{|c|} 
U.S. \\
Petroleum \\
Production \\
with Other \\
Inputs \\
2007-on \\
(dotted \\
line)
\end{tabular} & $\begin{array}{c}\text { U.S. } \\
\text { Petroleum } \\
\text { Production } \\
\text { without } \\
\text { Other } \\
\text { Inputs } \\
\text { 2007-on }\end{array}$ \\
\hline 1970 & 4.01 & 0.73 & 0.79 & 0.62 & 0.40 & 0.35 & 0.47 & 0.26 & 7.62 & 11.66 & 11.66 \\
\hline 1971 & 4.22 & 0.80 & 0.82 & 0.62 & 0.37 & 0.34 & 0.48 & 0.26 & 7.90 & 11.54 & 11.54 \\
\hline 1972 & 4.50 & 0.89 & 0.90 & 0.62 & 0.37 & 0.33 & 0.49 & 0.27 & 8.38 & 11.61 & 11.61 \\
\hline 1973 & 4.63 & 0.99 & 0.99 & 0.65 & 0.42 & 0.34 & 0.47 & 0.28 & 8.78 & 11.40 & 11.40 \\
\hline 1974 & 4.40 & 0.98 & 0.99 & 0.59 & 0.42 & 0.31 & 0.44 & 0.29 & 8.43 & 10.94 & 10.94 \\
\hline 1975 & 4.40 & 1.13 & 1.00 & 0.60 & 0.44 & 0.32 & 0.40 & 0.26 & 8.55 & 10.47 & 10.47 \\
\hline 1976 & 4.65 & 1.23 & 1.06 & 0.63 & 0.51 & 0.33 & 0.38 & 0.27 & 9.06 & 10.24 & 10.24 \\
\hline 1977 & 4.70 & 1.32 & 1.17 & 0.64 & 0.56 & 0.32 & 0.37 & 0.28 & 9.36 & 10.39 & 10.39 \\
\hline 1978 & 4.80 & 1.43 & 1.30 & 0.67 & 0.65 & 0.31 & 0.37 & 0.28 & 9.80 & 10.77 & 10.77 \\
\hline 1979 & 4.56 & 1.44 & 1.34 & 0.70 & 0.54 & 0.32 & 0.41 & 0.29 & 9.60 & 10.66 & 10.66 \\
\hline 1980 & 4.17 & 1.41 & 1.34 & 0.68 & 0.66 & 0.32 & 0.42 & 0.28 & 9.26 & 10.80 & 10.80 \\
\hline 1981 & 4.12 & 1.40 & 1.36 & 0.69 & 0.60 & 0.31 & 0.43 & 0.27 & 9.16 & 10.69 & 10.69 \\
\hline 1982 & 4.11 & 1.34 & 1.35 & 0.68 & 0.50 & 0.28 & 0.40 & 0.23 & 8.90 & 10.73 & 10.73 \\
\hline 1983 & 4.17 & 1.41 & 1.38 & 0.68 & 0.46 & 0.28 & 0.35 & 0.23 & 8.95 & 10.73 & 10.73 \\
\hline 1984 & 4.18 & 1.51 & 1.43 & 0.76 & 0.46 & 0.34 & 0.37 & 0.25 & 9.29 & 11.09 & 11.09 \\
\hline 1985 & 4.23 & 1.61 & 1.43 & 0.79 & 0.41 & 0.35 & 0.36 & 0.24 & 9.42 & 11.14 & 11.14 \\
\hline 1986 & 4.33 & 1.71 & 1.47 & 0.86 & 0.63 & 0.35 & 0.35 & 0.23 & 9.93 & 10.85 & 10.85 \\
\hline 1987 & 4.34 & 1.80 & 1.52 & 0.90 & 0.65 & 0.36 & 0.37 & 0.24 & 10.17 & 10.58 & 10.58 \\
\hline
\end{tabular}




\begin{tabular}{|c|c|c|c|c|c|c|c|c|c|c|c|}
\hline \multicolumn{12}{|c|}{$\begin{array}{c}\text { Historical and Future U.S. Petroleum Production and Transportation } \\
\text { Petroleum Use (Million barrels per day) }\end{array}$} \\
\hline Year & Autos & $\begin{array}{l}\text { Light } \\
\text { Trucks }\end{array}$ & $\begin{array}{c}\text { Medium } \\
\& \\
\text { Heavy } \\
\text { Trucks }\end{array}$ & Air & Water & $\begin{array}{c}\text { Off- } \\
\text { Highway }\end{array}$ & Rail & Pipeline & $\begin{array}{l}\text { Total } \\
\text { Trans- } \\
\text { portation }\end{array}$ & $\begin{array}{c}\text { U.S. } \\
\text { Petroleum } \\
\text { Production } \\
\text { with Other } \\
\text { Inputs } \\
\text { 2007-on } \\
\text { (dotted } \\
\text { line) }\end{array}$ & $\begin{array}{c}\text { U.S. } \\
\text { Petroleum } \\
\text { Production } \\
\text { without } \\
\text { Other } \\
\text { Inputs } \\
\text { 2007-on }\end{array}$ \\
\hline 1988 & 4.34 & 1.93 & 1.55 & 0.93 & 0.67 & 0.36 & 0.41 & 0.24 & 10.43 & 10.45 & 10.45 \\
\hline 1989 & 4.37 & 1.96 & 1.59 & 0.94 & 0.72 & 0.34 & 0.42 & 0.24 & 10.59 & 9.82 & 9.82 \\
\hline 1990 & 4.12 & 2.10 & 1.65 & 0.98 & 0.68 & 0.36 & 0.44 & 0.24 & 10.57 & 9.60 & 9.60 \\
\hline 1991 & 3.80 & 2.26 & 1.69 & 0.92 & 0.72 & 0.37 & 0.41 & 0.23 & 10.38 & 9.79 & 9.79 \\
\hline 1992 & 3.87 & 2.42 & 1.73 & 0.93 & 0.76 & 0.35 & 0.40 & 0.23 & 10.67 & 9.67 & 9.67 \\
\hline 1993 & 3.96 & 2.53 & 1.78 & 0.94 & 0.68 & 0.30 & 0.42 & 0.24 & 10.84 & 9.35 & 9.35 \\
\hline 1994 & 4.01 & 2.61 & 1.87 & 0.98 & 0.66 & 0.31 & 0.45 & 0.25 & 11.14 & 9.16 & 9.16 \\
\hline 1995 & 4.02 & 2.69 & 1.95 & 1.01 & 0.69 & 0.32 & 0.46 & 0.26 & 11.40 & 9.10 & 9.10 \\
\hline 1996 & 4.09 & 2.79 & 2.00 & 1.04 & 0.67 & 0.32 & 0.46 & 0.27 & 11.65 & 9.16 & 9.16 \\
\hline 1997 & 4.13 & 2.91 & 2.02 & 1.09 & 0.59 & 0.34 & 0.48 & 0.27 & 11.83 & 9.12 & 9.12 \\
\hline 1998 & 4.23 & 2.98 & 2.08 & 1.12 & 0.58 & 0.34 & 0.42 & 0.27 & 12.03 & 8.90 & 8.90 \\
\hline 1999 & 4.33 & 3.12 & 2.29 & 1.17 & 0.65 & 0.32 & 0.43 & 0.28 & 12.58 & 8.62 & 8.62 \\
\hline 2000 & 4.31 & 3.12 & 2.37 & 1.20 & 0.69 & 0.33 & 0.43 & 0.28 & 12.73 & 8.70 & 8.70 \\
\hline 2001 & 4.34 & 3.15 & 2.36 & 1.14 & 0.56 & 0.35 & 0.42 & 0.29 & 12.61 & 8.57 & 8.57 \\
\hline 2002 & 4.45 & 3.25 & 2.46 & 1.05 & 0.59 & 0.36 & 0.44 & 0.29 & 12.88 & 8.58 & 8.58 \\
\hline 2003 & 4.39 & 3.57 & 2.40 & 1.05 & 0.51 & 0.36 & 0.40 & 0.29 & 12.96 & 8.37 & 8.37 \\
\hline 2004 & 4.43 & 3.71 & 2.23 & 1.11 & 0.61 & 0.37 & 0.39 & 0.31 & 13.16 & 8.30 & 8.30 \\
\hline 2005 & 4.54 & 3.45 & 2.49 & 1.17 & 0.65 & 0.38 & 0.40 & 0.31 & 13.38 & 7.89 & 7.89 \\
\hline 2006 & 4.43 & 3.57 & 2.54 & 1.19 & 0.69 & 0.38 & 0.40 & 0.32 & 13.49 & 7.84 & 7.84 \\
\hline 2007 & 4.38 & 3.63 & 3.15 & 1.19 & 0.74 & 0.38 & 0.42 & 0.31 & 14.18 & 7.84 & 7.84 \\
\hline
\end{tabular}




\begin{tabular}{|c|c|c|c|c|c|c|c|c|c|c|c|}
\hline \multicolumn{12}{|c|}{$\begin{array}{c}\text { Historical and Future U.S. Petroleum Production and Transportation } \\
\text { Petroleum Use (Million barrels per day) }\end{array}$} \\
\hline Year & Autos & $\begin{array}{l}\text { Light } \\
\text { Trucks }\end{array}$ & $\begin{array}{c}\text { Medium } \\
\& \\
\text { Heavy } \\
\text { Trucks }\end{array}$ & Air & Water & $\begin{array}{c}\text { Off- } \\
\text { Highway }\end{array}$ & Rail & Pipeline & $\begin{array}{l}\text { Total } \\
\text { Trans- } \\
\text { portation }\end{array}$ & $\begin{array}{c}\text { U.S. } \\
\text { Petroleum } \\
\text { Production } \\
\text { with Other } \\
\text { Inputs } \\
\text { 2007-on } \\
\text { (dotted } \\
\text { line) }\end{array}$ & $\begin{array}{c}\text { U.S. } \\
\text { Petroleum } \\
\text { Production } \\
\text { without } \\
\text { Other } \\
\text { Inputs } \\
\text { 2007-on }\end{array}$ \\
\hline 2008 & 4.20 & 3.58 & 3.18 & 1.13 & 0.65 & 0.38 & 0.43 & 0.30 & 13.84 & 7.75 & 7.75 \\
\hline 2009 & 4.19 & 3.59 & 2.97 & 1.01 & 0.61 & 0.38 & 0.41 & 0.26 & 13.41 & 8.18 & 8.18 \\
\hline 2010 & 4.07 & 4.91 & 2.25 & 1.29 & 0.59 & 0.42 & 0.26 & 0.43 & 14.22 & 9.51 & 8.50 \\
\hline 2011 & 4.05 & 4.96 & 2.36 & 1.25 & 0.59 & 0.43 & 0.27 & 0.42 & 14.32 & 9.64 & 8.41 \\
\hline 2012 & 4.04 & 5.04 & 2.44 & 1.26 & 0.59 & 0.44 & 0.28 & 0.42 & 14.50 & 9.79 & 8.55 \\
\hline 2013 & 4.05 & 5.08 & 2.55 & 1.26 & 0.59 & 0.45 & 0.28 & 0.42 & 14.67 & 10.05 & 8.77 \\
\hline 2014 & 4.05 & 5.10 & 2.56 & 1.28 & 0.59 & 0.45 & 0.29 & 0.42 & 14.74 & 10.27 & 8.96 \\
\hline 2015 & 4.06 & 5.11 & 2.60 & 1.30 & 0.59 & 0.45 & 0.30 & 0.42 & 14.83 & 10.47 & 9.05 \\
\hline 2016 & 4.09 & 5.11 & 2.63 & 1.31 & 0.59 & 0.45 & 0.30 & 0.42 & 14.90 & 10.71 & 9.21 \\
\hline 2017 & 4.11 & 5.11 & 2.66 & 1.32 & 0.60 & 0.46 & 0.31 & 0.42 & 14.99 & 10.97 & 9.35 \\
\hline 2018 & 4.14 & 5.08 & 2.69 & 1.34 & 0.60 & 0.47 & 0.31 & 0.42 & 15.05 & 11.11 & 9.41 \\
\hline 2019 & 4.18 & 5.04 & 2.72 & 1.35 & 0.60 & 0.48 & 0.31 & 0.42 & 15.10 & 11.22 & 9.45 \\
\hline 2020 & 4.23 & 5.01 & 2.75 & 1.36 & 0.60 & 0.48 & 0.32 & 0.42 & 15.16 & 11.32 & 9.46 \\
\hline 2021 & 4.28 & 4.95 & 2.78 & 1.38 & 0.60 & 0.48 & 0.32 & 0.42 & 15.20 & 11.33 & 9.49 \\
\hline 2022 & 4.35 & 4.91 & 2.81 & 1.39 & 0.62 & 0.48 & 0.33 & 0.42 & 15.31 & 11.56 & 9.56 \\
\hline 2023 & 4.42 & 4.89 & 2.84 & 1.40 & 0.62 & 0.49 & 0.33 & 0.40 & 15.40 & 11.73 & 9.59 \\
\hline 2024 & 4.51 & 4.87 & 2.87 & 1.41 & 0.62 & 0.50 & 0.33 & 0.40 & 15.51 & 11.85 & 9.54 \\
\hline 2025 & 4.60 & 4.84 & 2.90 & 1.42 & 0.62 & 0.50 & 0.34 & 0.40 & 15.62 & 11.88 & 9.48 \\
\hline 2026 & 4.67 & 4.82 & 2.93 & 1.43 & 0.62 & 0.50 & 0.34 & 0.40 & 15.72 & 11.91 & 9.43 \\
\hline 2027 & 4.75 & 4.79 & 2.95 & 1.44 & 0.62 & 0.51 & 0.34 & 0.40 & 15.80 & 11.99 & 9.41 \\
\hline
\end{tabular}




\begin{tabular}{|l|l|l|l|l|l|l|l|l|l|}
\hline \multicolumn{2}{|c|}{ Historical and Future U.S. Petroleum Production and Transportation } \\
\hline
\end{tabular}




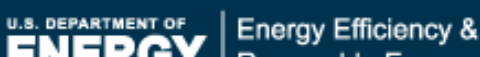 \\ Renewable Energy}

\section{Vehicle Technologies Program}

\section{Fact \#688: August 15, 2011 All Sectors' Petroleum Gap}

Before1989 the U.S. produced enough petroleum to meet the needs of the transportation sector, but was still short of meeting the petroleum needs of all the sectors, including industrial, residential and commercial, and electric utilities. In 1973 the gap between what the U.S. produced and what was consumed was 5.6 million barrels per day. By 2035, the gap is expected to be at least 9.6 million barrels per day if all sources of petroleum are included or 12.7 million barrels per day if only conventional petroleum sources are used.

U.S. Petroleum Production and Consumption, 1970-2035

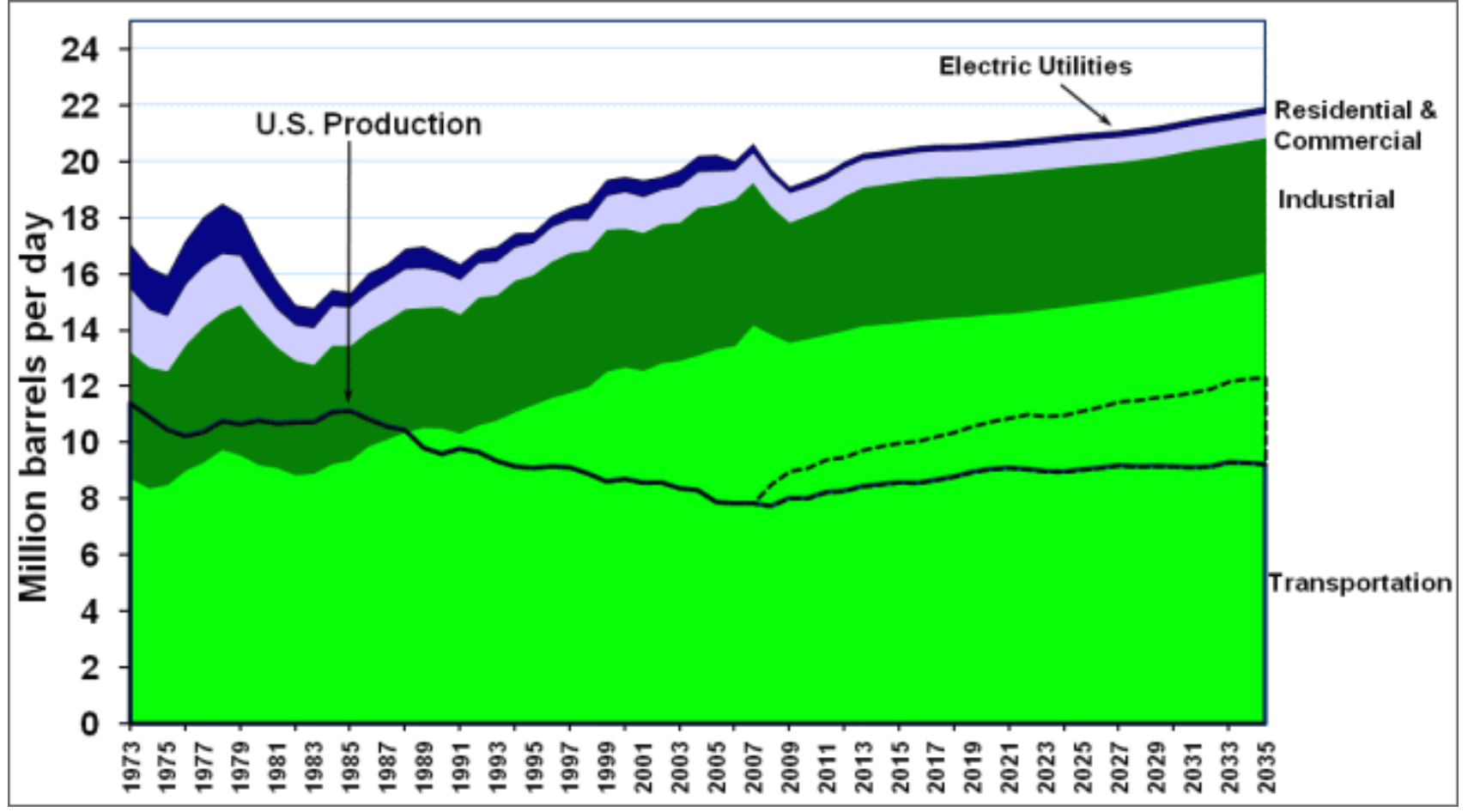

Notes:

- The U.S. Production has two lines after 2005. The solid line is conventional sources of petroleum. The dashed line adds in other non-petroleum sources, including ethanol, biomass, liquids from coal, other blending components, other hydrocarbons, and ethers.

- Between 2009 and 2010 the data change from historical to projected values.

- The sharp increase in transportation value between 2006 and 2007 is due to the Federal Highway Administration methodology change in heavy truck fuel use. 


\section{Supporting Information}

\begin{tabular}{|c|c|c|c|c|c|c|c|}
\hline \multicolumn{8}{|c|}{$\begin{array}{l}\text { Historical and Future U.S. Petroleum Production and Petroleum } \\
\text { Use (Million barrels per day) }\end{array}$} \\
\hline Year & Transportation & Industrial & $\begin{array}{l}\text { Residential } \\
\text { and } \\
\text { Commercial }\end{array}$ & $\begin{array}{l}\text { Electric } \\
\text { Utilities }\end{array}$ & Total & $\begin{array}{l}\text { U.S. } \\
\text { Petroleum } \\
\text { Production } \\
\text { with Other } \\
\text { Inputs } \\
\text { 2007-on } \\
\text { (dotted } \\
\text { line) }\end{array}$ & $\begin{array}{l}\text { U.S. } \\
\text { Petroleum } \\
\text { Production } \\
\text { without } \\
\text { Other } \\
\text { Inputs } \\
\text { 2007-on }\end{array}$ \\
\hline 1973 & 8.78 & 4.48 & 2.23 & 1.54 & 17.04 & 11.40 & 11.40 \\
\hline 1973 & 8.78 & 4.48 & 2.23 & 1.54 & 17.03 & 11.40 & 11.40 \\
\hline 1974 & 8.43 & 4.30 & 2.04 & 1.48 & 16.24 & 10.94 & 10.94 \\
\hline 1975 & 8.55 & 4.04 & 1.95 & 1.39 & 15.92 & 10.47 & 10.47 \\
\hline 1976 & 9.06 & 4.46 & 2.13 & 1.52 & 17.17 & 10.24 & 10.24 \\
\hline 1977 & 9.36 & 4.82 & 2.14 & 1.71 & 18.03 & 10.39 & 10.39 \\
\hline 1978 & 9.80 & 4.87 & 2.07 & 1.75 & 18.49 & 10.77 & 10.77 \\
\hline 1979 & 9.60 & 5.34 & 1.73 & 1.44 & 18.11 & 10.66 & 10.66 \\
\hline 1980 & 9.26 & 4.86 & 1.52 & 1.15 & 16.79 & 10.80 & 10.80 \\
\hline 1981 & 9.16 & 4.27 & 1.33 & 0.96 & 15.73 & 10.69 & 10.69 \\
\hline 1982 & 8.90 & 4.06 & 1.24 & 0.69 & 14.89 & 10.73 & 10.73 \\
\hline 1983 & 8.95 & 3.85 & 1.29 & 0.68 & 14.78 & 10.73 & 10.73 \\
\hline 1984 & 9.29 & 4.20 & 1.38 & 0.56 & 15.44 & 11.09 & 11.09 \\
\hline 1985 & 9.42 & 4.07 & 1.34 & 0.48 & 15.31 & 11.14 & 11.14 \\
\hline 1986 & 9.93 & 4.09 & 1.37 & 0.64 & 16.02 & 10.85 & 10.85 \\
\hline 1987 & 10.17 & 4.21 & 1.40 & 0.55 & 16.33 & 10.58 & 10.58 \\
\hline 1988 & 10.43 & 4.36 & 1.41 & 0.69 & 16.89 & 10.45 & 10.45 \\
\hline 1989 & 10.59 & 4.25 & 1.39 & 0.75 & 16.97 & 9.82 & 9.82 \\
\hline 1990 & 10.57 & 4.30 & 1.23 & 0.57 & 16.67 & 9.60 & 9.60 \\
\hline 1991 & 10.38 & 4.22 & 1.21 & 0.53 & 16.33 & 9.79 & 9.79 \\
\hline 1992 & 10.67 & 4.53 & 1.20 & 0.44 & 16.84 & 9.67 & 9.67 \\
\hline 1993 & 10.84 & 4.44 & 1.18 & 0.49 & 16.96 & 9.35 & 9.35 \\
\hline 1994 & 11.14 & 4.67 & 1.17 & 0.47 & 17.44 & 9.16 & 9.16 \\
\hline 1995 & 11.40 & 4.59 & 1.13 & 0.33 & 17.46 & 9.10 & 9.10 \\
\hline 1996 & 11.65 & 4.83 & 1.21 & 0.36 & 18.05 & 9.16 & 9.16 \\
\hline 1997 & 11.83 & 4.95 & 1.16 & 0.41 & 18.35 & 9.12 & 9.12 \\
\hline
\end{tabular}




\begin{tabular}{|c|c|c|c|c|c|c|c|}
\hline \multicolumn{8}{|c|}{$\begin{array}{l}\text { Historical and Future U.S. Petroleum Production and Petroleum } \\
\text { Use (Million barrels per day) }\end{array}$} \\
\hline Year & Transportation & Industrial & $\begin{array}{l}\text { Residential } \\
\text { and } \\
\text { Commercial }\end{array}$ & $\begin{array}{l}\text { Electric } \\
\text { Utilities }\end{array}$ & Total & $\begin{array}{c}\text { U.S. } \\
\text { Petroleum } \\
\text { Production } \\
\text { with Other } \\
\text { Inputs } \\
\text { 2007-on } \\
\text { (dotted } \\
\text { line) }\end{array}$ & $\begin{array}{c}\text { U.S. } \\
\text { Petroleum } \\
\text { Production } \\
\text { without } \\
\text { Other } \\
\text { Inputs } \\
\text { 2007-on }\end{array}$ \\
\hline 1998 & 12.03 & 4.84 & 1.08 & 0.58 & 18.53 & 8.90 & 8.90 \\
\hline 1999 & 12.58 & 5.03 & 1.18 & 0.53 & 19.33 & 8.62 & 8.62 \\
\hline 2000 & 12.73 & 4.92 & 1.28 & 0.51 & 19.44 & 8.70 & 8.70 \\
\hline 2001 & 12.61 & 4.89 & 1.25 & 0.56 & 19.32 & 8.57 & 8.57 \\
\hline 2002 & 12.88 & 4.93 & 1.19 & 0.43 & 19.43 & 8.58 & 8.58 \\
\hline 2003 & 12.96 & 4.90 & 1.28 & 0.53 & 19.67 & 8.37 & 8.37 \\
\hline 2004 & 13.16 & 5.23 & 1.26 & 0.54 & 20.18 & 8.30 & 8.30 \\
\hline 2005 & 13.38 & 5.10 & 1.20 & 0.55 & 20.22 & 7.88 & 7.88 \\
\hline 2006 & 13.49 & 5.19 & 1.03 & 0.29 & 19.99 & 7.83 & 7.83 \\
\hline 2007 & 14.23 & 5.05 & 1.04 & 0.29 & 20.62 & 7.84 & 7.84 \\
\hline 2008 & 13.89 & 4.53 & 1.06 & 0.21 & 19.69 & 8.52 & 7.74 \\
\hline 2009 & 13.61 & 4.250 & 1.04 & 0.180 & 19.08 & 8.99 & 8.03 \\
\hline 2010 & 13.74 & 4.370 & 1.00 & 0.200 & 19.31 & 9.11 & 8.02 \\
\hline 2011 & 13.88 & 4.500 & 1.00 & 0.200 & 19.58 & 9.41 & 8.24 \\
\hline 2012 & 14.03 & 4.770 & 0.99 & 0.190 & 19.98 & 9.49 & 8.28 \\
\hline 2013 & 14.20 & 4.910 & 0.97 & 0.190 & 20.27 & 9.75 & 8.45 \\
\hline 2014 & 14.25 & 4.950 & 0.96 & 0.190 & 20.35 & 9.89 & 8.52 \\
\hline 2015 & 14.31 & 4.990 & 0.95 & 0.190 & 20.44 & 10.00 & 8.58 \\
\hline 2016 & 14.39 & 5.010 & 0.94 & 0.190 & 20.53 & 10.07 & 8.57 \\
\hline 2017 & 14.45 & 5.010 & 0.93 & 0.190 & 20.58 & 10.23 & 8.67 \\
\hline 2018 & 14.50 & 4.980 & 0.92 & 0.190 & 20.59 & 10.39 & 8.79 \\
\hline 2019 & 14.54 & 4.970 & 0.92 & 0.190 & 20.62 & 10.60 & 8.96 \\
\hline 2020 & 14.61 & 4.960 & 0.91 & 0.200 & 20.68 & 10.77 & 9.06 \\
\hline 2021 & 14.64 & 4.980 & 0.90 & 0.200 & 20.72 & 10.89 & 9.10 \\
\hline 2022 & 14.71 & 4.980 & 0.90 & 0.200 & 20.79 & 11.02 & 9.05 \\
\hline
\end{tabular}




\begin{tabular}{l} 
Historical and Future U.S. Petroleum Production and Petroleum \\
Use (Million barrels per day) \\
\hline \\
\end{tabular}




\section{u.s. ofpaatment of | Energy Efficiency \&}

\section{Vehicle Technologies Program}

\section{Fact \#689: August 22, 2011 Energy Use by Sector and Source}

The transportation sector consumed $28 \%$ of U.S. energy in 2010 , nearly all of it (93.5\%) in petroleum use. The industrial sector used about $40 \%$ petroleum and $40 \%$ natural gas. The electric utility sector used little petroleum, but was dependent on coal for nearly half of the energy it consumed. Renewables, such as biofuels for transportation, were being used in every sector in 2010.
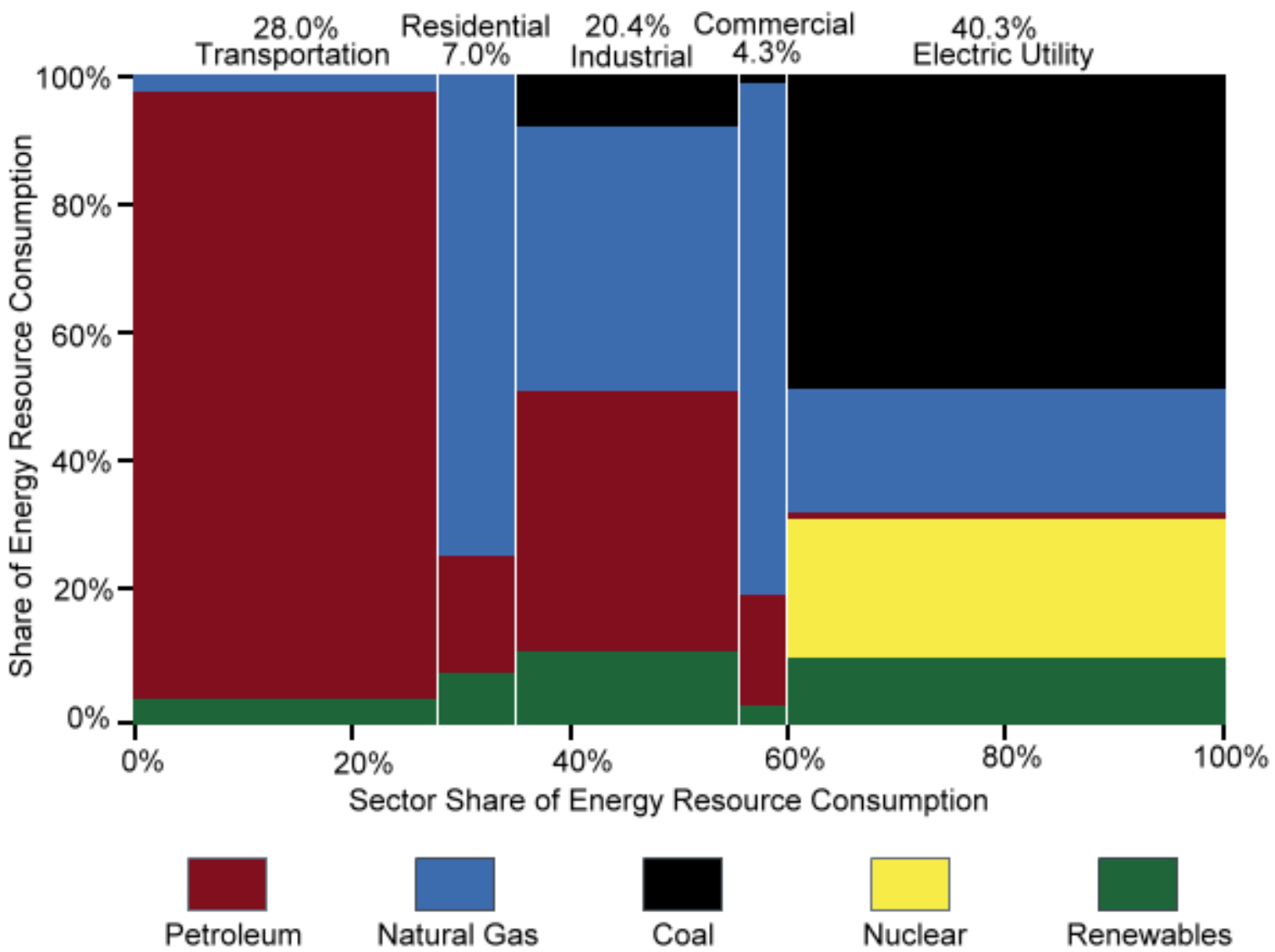


\section{Supporting Information}

\begin{tabular}{|c|c|c|c|c|c|}
\hline \multicolumn{6}{|c|}{ Energy Consumption by Sector and Source: 2010} \\
\hline & Transportation & Residential & Commercial & Industrial & \begin{tabular}{|c} 
Electric \\
Utility
\end{tabular} \\
\hline & \multicolumn{5}{|c|}{ Total Energy Share } \\
\hline & $28.0 \%$ & $7.0 \%$ & $4.3 \%$ & $20.4 \%$ & $40.4 \%$ \\
\hline & \multicolumn{5}{|c|}{ Energy Share by Source } \\
\hline Coal & $0.0 \%$ & $0.1 \%$ & $1.4 \%$ & $8.1 \%$ & $48.3 \%$ \\
\hline Natural Gas & $2.5 \%$ & $74.0 \%$ & $78.5 \%$ & $40.6 \%$ & $19.0 \%$ \\
\hline Petroleum & $93.5 \%$ & $17.8 \%$ & $17.1 \%$ & $40.1 \%$ & $1.0 \%$ \\
\hline Renewables & $4.0 \%$ & $8.1 \%$ & $3.0 \%$ & $11.3 \%$ & $10.2 \%$ \\
\hline Nuclear & $0.0 \%$ & $0.0 \%$ & $0.0 \%$ & $0.0 \%$ & $21.3 \%$ \\
\hline \multirow[t]{3}{*}{ Total } & $100.0 \%$ & $100.0 \%$ & $100.0 \%$ & $100.0 \%$ & $100.0 \%$ \\
\hline & \multicolumn{5}{|c|}{ Primary Energy Use in Quadrillion Btu } \\
\hline & 27.4 & 6.8 & 4.2 & 20.0 & 39.6 \\
\hline $\begin{array}{l}\text { Source: } \\
\text { Source: U.S. } \\
\text { [Original sourc }\end{array}$ & $\begin{array}{l}\text { epartment of Energ } \\
\text { e: Energy Informatic }\end{array}$ & $\frac{2010 \text { Vehicle }}{\text { Administratic }}$ & $\frac{\text { Technologies }}{\text { on, Monthly En }}$ & $\frac{\text { larket Repor }}{\text { gy Review.] }}$ & \\
\hline
\end{tabular}




\section{u.s. oppaprusur of | Energy Efficiency \& \\ Renewable Energy}

\section{Vehicle Technologies Program}

\section{Fact \#690: August 29, 2011 Characteristics of New Light Vehicles over Time}

From model year (MY) 1980 to 2010, there have been significant gains made in automotive technology. For new light vehicles, horsepower has more than doubled and "0-to-60" times have dropped from 14.3 to 9.5 seconds. The average weight grew to a high of 4,111 pounds in 2004 and has dropped slightly since then (4,009 pounds in 2010). The average fuel economy of new light vehicles has gradually fluctuated higher and lower over the years, with fuel economy in 2010 being just above the 1987 average.

Characteristics of New Light Vehicles Sold, MY 1980-2010

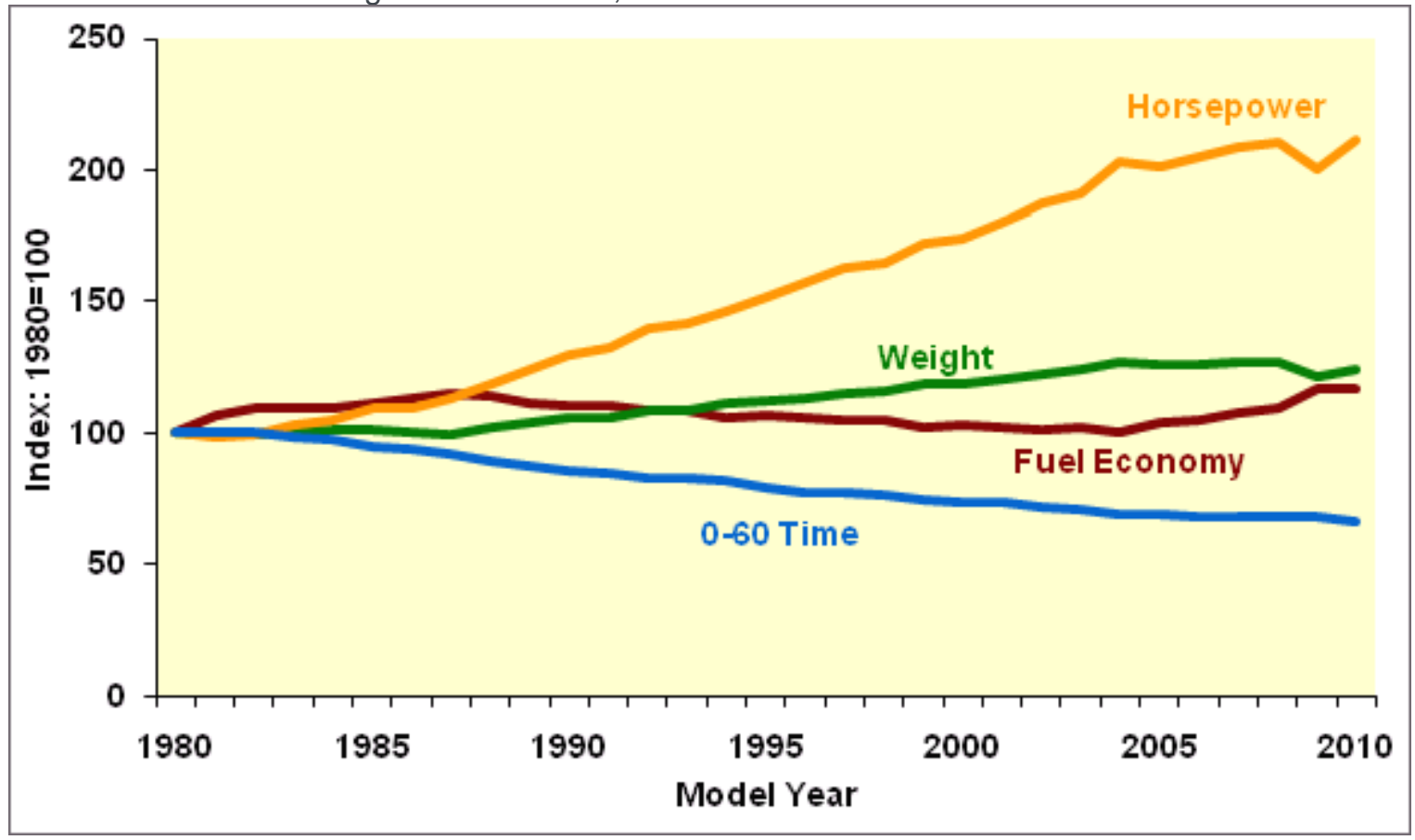




\section{Supporting Information}

\begin{tabular}{|c|c|c|c|c|}
\hline \multicolumn{5}{|c|}{$\begin{array}{l}\text { Characteristics of New Light Vehicles Sold, MY 1980- } \\
\qquad 2010\end{array}$} \\
\hline $\begin{array}{l}\text { Model } \\
\text { Year }\end{array}$ & MPG & Weight & Horsepower & $\begin{array}{l}0-60 \\
\text { Time }\end{array}$ \\
\hline 1980 & 19.2 & 3,228 & 104 & 14.3 \\
\hline 1981 & 20.5 & 3,202 & 102 & 14.4 \\
\hline 1982 & 21.1 & 3,202 & 103 & 14.4 \\
\hline 1983 & 21.0 & 3,257 & 107 & 14.1 \\
\hline 1984 & 21.0 & 3,262 & 109 & 14.0 \\
\hline 1985 & 21.3 & 3,271 & 114 & 13.5 \\
\hline 1986 & 21.8 & 3,238 & 114 & 13.4 \\
\hline 1987 & 22.0 & 3,221 & 118 & 13.1 \\
\hline 1988 & 21.9 & 3,283 & 123 & 12.8 \\
\hline 1989 & 21.4 & 3,351 & 129 & 12.5 \\
\hline 1990 & 21.2 & 3,426 & 135 & 12.2 \\
\hline 1991 & 21.2 & 3,410 & 138 & 12.1 \\
\hline 1992 & 20.8 & 3,512 & 145 & 11.8 \\
\hline 1993 & 20.9 & 3,519 & 147 & 11.8 \\
\hline 1994 & 20.4 & 3,603 & 152 & 11.7 \\
\hline 1995 & 20.5 & 3,613 & 158 & 11.3 \\
\hline 1996 & 20.4 & 3,659 & 164 & 11.1 \\
\hline 1997 & 20.1 & 3,727 & 169 & 11.0 \\
\hline 1998 & 20.1 & 3,744 & 171 & 10.9 \\
\hline 1999 & 19.7 & 3,835 & 179 & 10.7 \\
\hline 2000 & 19.8 & 3,821 & 181 & 10.6 \\
\hline 2001 & 19.6 & 3,879 & 187 & 10.5 \\
\hline 2002 & 19.4 & 3,951 & 195 & 10.3 \\
\hline 2003 & 19.6 & 3,999 & 199 & 10.2 \\
\hline 2004 & 19.3 & 4,111 & 211 & 9.9 \\
\hline 2005 & 19.9 & 4,059 & 209 & 9.9 \\
\hline
\end{tabular}




\begin{tabular}{|c|c|c|c|c|}
\hline \multicolumn{5}{|c|}{$\begin{array}{l}\text { Characteristics of New Light Vehicles Sold, MY } 1980 \\
\qquad 2010\end{array}$} \\
\hline $\begin{array}{l}\text { Model } \\
\text { Year }\end{array}$ & MPG & Weight & Horsepower & $\begin{array}{l}0-60 \\
\text { Time }\end{array}$ \\
\hline 2006 & 20.1 & 4,067 & 213 & 9.8 \\
\hline 2007 & 20.6 & 4,093 & 217 & 9.7 \\
\hline 2008 & 21.0 & 4,085 & 219 & 9.7 \\
\hline 2009 & 22.4 & 3,917 & 208 & 9.7 \\
\hline 2010 & 22.5 & 4,009 & 220 & 9.5 \\
\hline \multicolumn{5}{|c|}{$\begin{array}{l}\text { Source: } \\
\text { U.S. Department of Energy, } 2010 \text { Vehicle Technologies } \\
\text { Market Report } \\
\text { Protection Agency, Light-Duty Automotive Technology, } \\
\text { Carbon Dioxide Emissions, and Fuel Economy Trends: } 1975 \\
\text { Through 2010] }\end{array}$} \\
\hline
\end{tabular}




\section{u.s. oppaprusur of |Energy Efficiency \& \\ Renewable Energy}

\section{Vehicle Technologies Program}

\section{Fact \#691: September 5, 2011}

\section{Mexico Surpassed Canada in Vehicle Production}

In 2008, Mexico produced more vehicles than Canada for the first time. In 2010, the gap widened. Seven automakers operate 12 assembly plants in Mexico. As a result, some major automotive suppliers are locating facilities in Mexico as well. U.S. production of vehicles fell to a low of 5.6 million vehicles in 2009, but rose in 2010 .

North American Vehicle Production by Country, 1990-2010

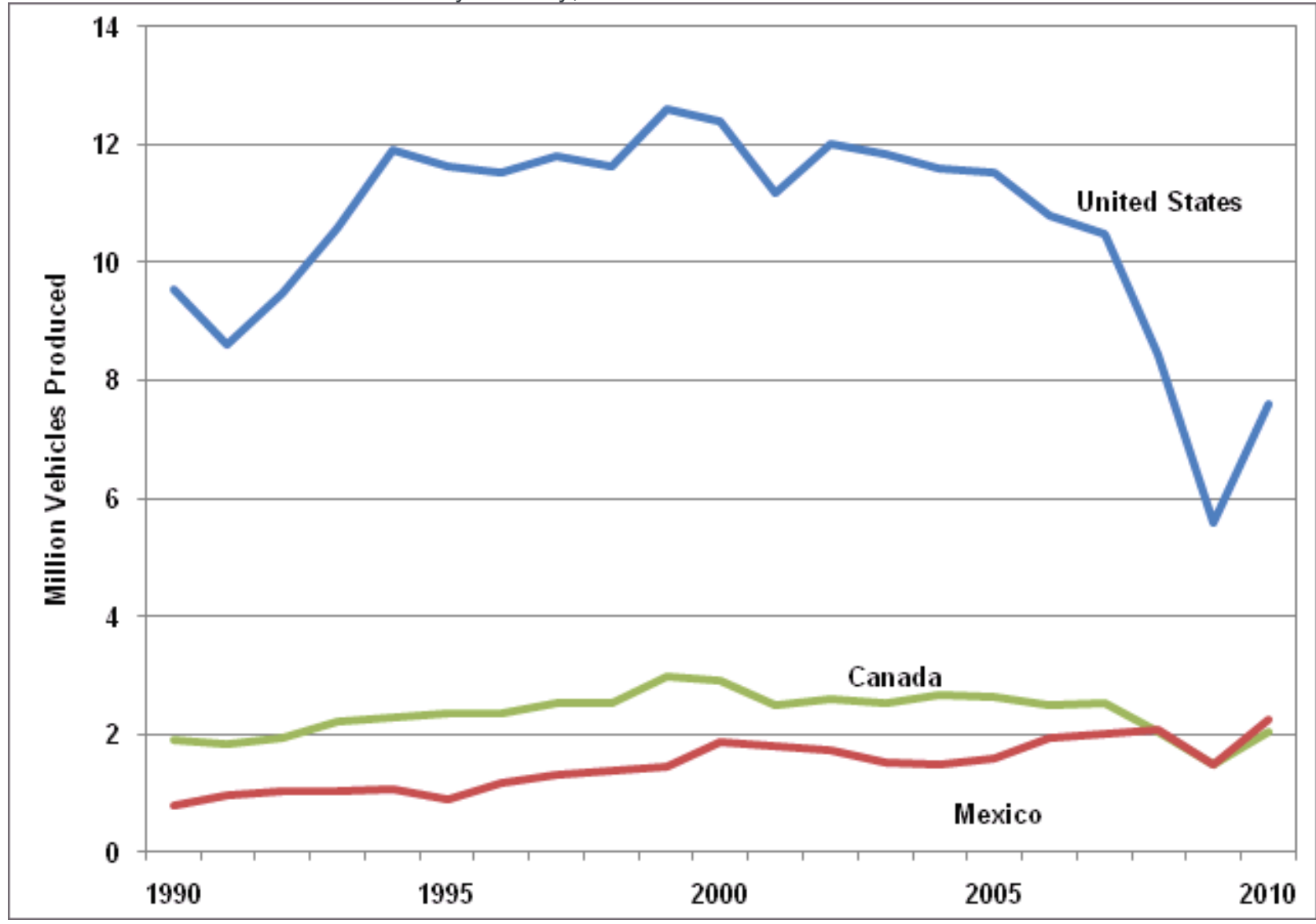




\section{Supporting Information}

\begin{tabular}{|c|c|c|c|}
\hline \multicolumn{4}{|c|}{$\begin{array}{l}\text { North American Vehicle Production by Country, } \\
\qquad 1990-2010\end{array}$} \\
\hline Calendar Year & $\begin{array}{l}\text { United } \\
\text { States }\end{array}$ & Canada & Mexico \\
\hline 1990 & 9.5 & 1.9 & 0.8 \\
\hline 1991 & 8.6 & 1.9 & 1.0 \\
\hline 1992 & 9.5 & 1.9 & 1.0 \\
\hline 1993 & 10.6 & 2.2 & 1.0 \\
\hline 1994 & 11.9 & 2.3 & 1.1 \\
\hline 1995 & 11.6 & 2.4 & 0.9 \\
\hline 1996 & 11.5 & 2.4 & 1.2 \\
\hline 1997 & 11.8 & 2.5 & 1.3 \\
\hline 1998 & 11.6 & 2.5 & 1.4 \\
\hline 1999 & 12.6 & 3.0 & 1.4 \\
\hline 2000 & 12.4 & 2.9 & 1.9 \\
\hline 2001 & 11.2 & 2.5 & 1.8 \\
\hline 2002 & 12.0 & 2.6 & 1.8 \\
\hline 2003 & 11.8 & 2.5 & 1.5 \\
\hline 2004 & 11.6 & 2.7 & 1.5 \\
\hline 2005 & 11.5 & 2.6 & 1.6 \\
\hline 2006 & 10.8 & 2.5 & 2.0 \\
\hline 2007 & 10.5 & 2.5 & 2.0 \\
\hline 2008 & 8.4 & 2.0 & 2.1 \\
\hline 2009 & 5.6 & 1.5 & 1.5 \\
\hline 2010 & 7.6 & 2.1 & 2.3 \\
\hline $\begin{array}{l}\text { Sources: Crain C } \\
\text { 2011, and Ward's }\end{array}$ & icatio & otive $N$ & June 27, \\
\hline
\end{tabular}




\section{U.s. ofpartment of | Energy Efficiency \& \\ Renewable Energy}

\section{Vehicle Technologies Program}

\section{Fact \#692: September 12, 2011 Fuel Economy Distribution for New Cars and Light Trucks}

Nearly 64\% of new cars sold in model year (MY) 1975 had combined highway/city fuel economy of 15 miles per gallon (mpg) or less [blue shading]. By 2010,63\% of cars had fuel economy of $25 \mathrm{mpg}$ or higher [green shading and up]. Light trucks, which typically have lower fuel economy than cars, show a drastic change in the market in the early 1980's - moving towards new light trucks with 15-20 mpg. While the majority of light trucks sold are still in the 15-20 mpg range, more than 37\% in 2009 and 2010 were 20 mpg or higher.

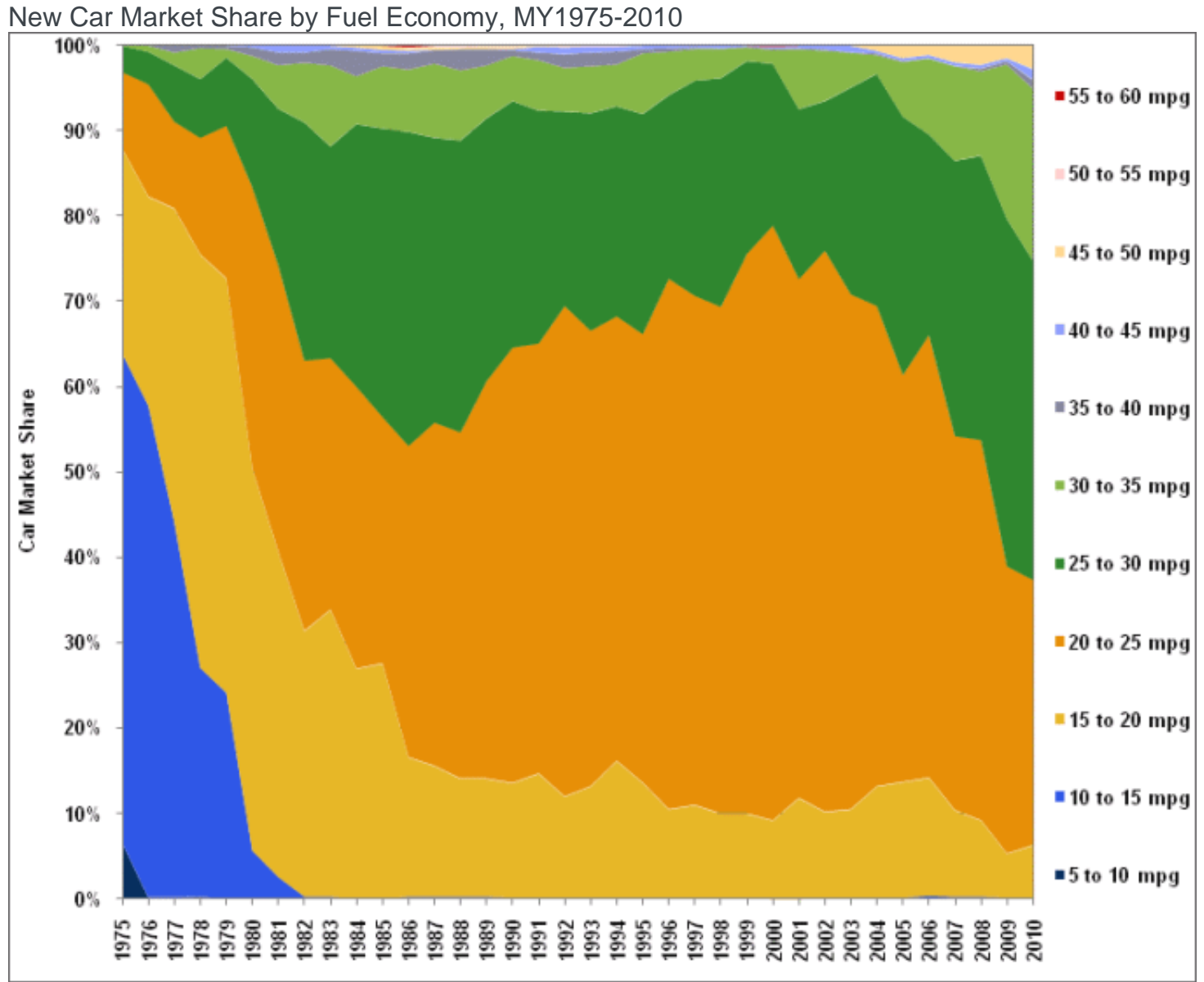


New Light Truck Market Share by Fuel Economy, MY1975-2010

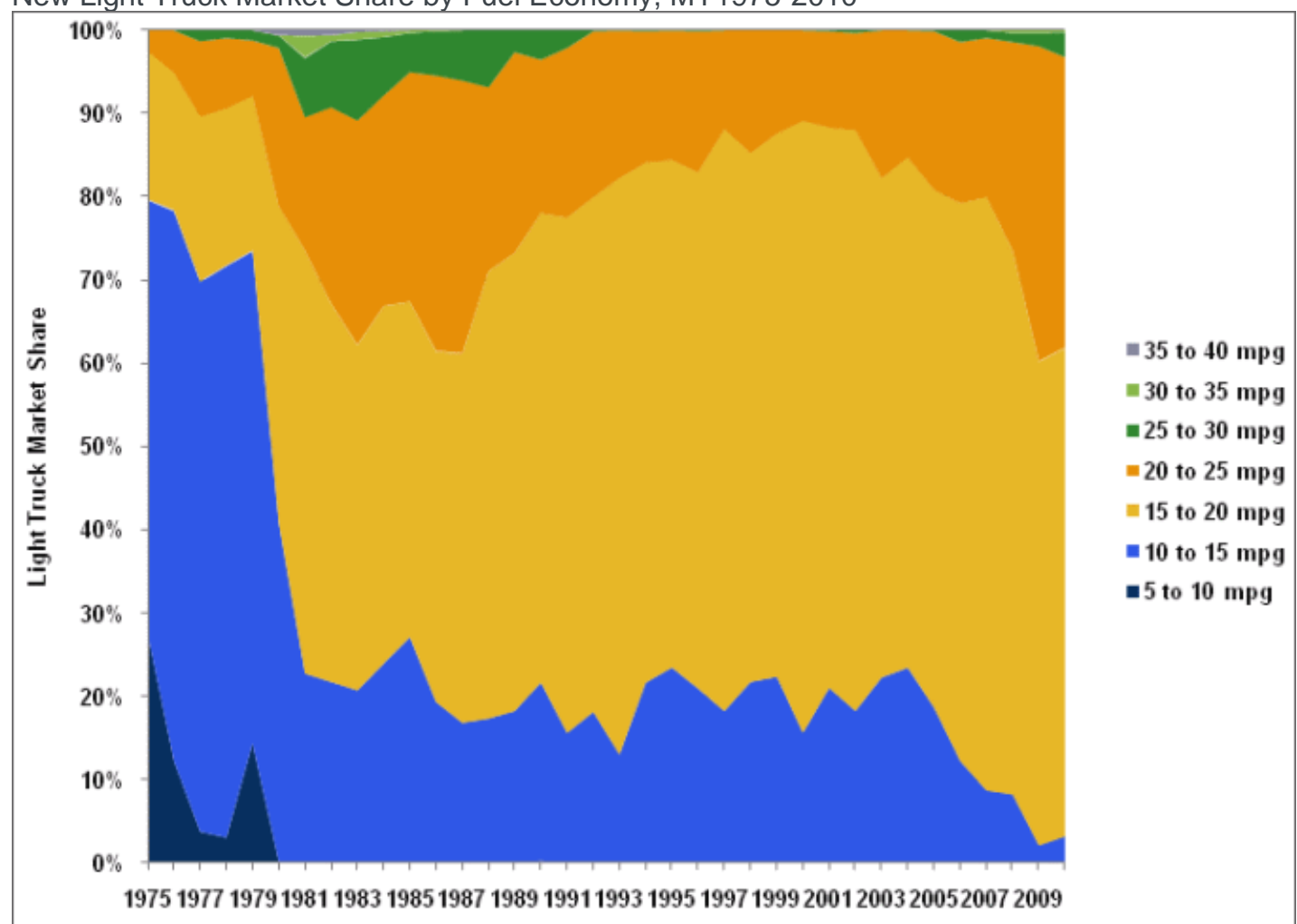

\section{Supporting Information}

\begin{tabular}{|c|c|c|c|c|c|c|c|c|c|c|c|}
\hline \multicolumn{12}{|c|}{ Car Market Share } \\
\hline $\begin{array}{l}\text { Model } \\
\text { Year }\end{array}$ & $\begin{array}{l}\text { 5-10 } \\
\mathrm{mpg}\end{array}$ & $\begin{array}{c}\text { 10-15 } \\
\text { mpg }\end{array}$ & $\begin{array}{c}15-20 \\
\mathrm{mpg}\end{array}$ & $\begin{array}{c}20-25 \\
\mathrm{mpg}\end{array}$ & $\begin{array}{c}25-30 \\
\mathrm{mpg}\end{array}$ & $\begin{array}{c}30-35 \\
\mathrm{mpg}\end{array}$ & $\begin{array}{c}35- \\
40 \\
\mathrm{mpg}\end{array}$ & $\begin{array}{c}40- \\
45 \\
\mathrm{mpg}\end{array}$ & $\begin{array}{c}45- \\
50 \\
\mathrm{mpg}\end{array}$ & $\begin{array}{c}50- \\
55 \\
\mathrm{mpg}\end{array}$ & $\begin{array}{c}55- \\
60 \\
\mathrm{mpg}\end{array}$ \\
\hline 1975 & $6.6 \%$ & $57.3 \%$ & $23.9 \%$ & $9.0 \%$ & $3.1 \%$ & & & & & & \\
\hline 1976 & $0.2 \%$ & $57.6 \%$ & $24.4 \%$ & $13.2 \%$ & $3.8 \%$ & $0.7 \%$ & & & & & \\
\hline 1977 & $0.2 \%$ & $44.0 \%$ & $36.7 \%$ & $10.2 \%$ & $6.6 \%$ & $1.5 \%$ & $0.8 \%$ & & & & \\
\hline 1978 & $0.3 \%$ & $26.8 \%$ & $48.4 \%$ & $13.7 \%$ & $6.9 \%$ & $3.6 \%$ & $0.3 \%$ & & & & \\
\hline 1979 & $0.0 \%$ & $24.2 \%$ & $48.5 \%$ & $17.9 \%$ & $8.0 \%$ & $1.0 \%$ & $0.4 \%$ & & & & \\
\hline 1980 & $0.0 \%$ & $5.7 \%$ & $44.7 \%$ & $33.0 \%$ & $12.7 \%$ & $2.7 \%$ & $1.0 \%$ & $0.2 \%$ & & & \\
\hline 1981 & $0.0 \%$ & $2.6 \%$ & $38.1 \%$ & $33.6 \%$ & $18.4 \%$ & $5.1 \%$ & $1.5 \%$ & $0.8 \%$ & & & \\
\hline 1982 & $0.0 \%$ & $0.3 \%$ & $31.1 \%$ & $31.7 \%$ & $27.9 \%$ & $7.0 \%$ & $1.2 \%$ & $0.8 \%$ & & & \\
\hline 1983 & $0.0 \%$ & $0.3 \%$ & $33.6 \%$ & $29.5 \%$ & $24.8 \%$ & $9.5 \%$ & $1.9 \%$ & $0.4 \%$ & $0.0 \%$ & & \\
\hline 1984 & $0.0 \%$ & $0.1 \%$ & $26.9 \%$ & $33.1 \%$ & $30.8 \%$ & $5.6 \%$ & $3.0 \%$ & $0.4 \%$ & $0.2 \%$ & & \\
\hline 1985 & $0.0 \%$ & $0.1 \%$ & $27.5 \%$ & $28.9 \%$ & $33.8 \%$ & $7.3 \%$ & $1.5 \%$ & $0.4 \%$ & $0.5 \%$ & & \\
\hline
\end{tabular}




\begin{tabular}{|c|c|c|c|c|c|c|c|c|c|c|c|}
\hline \multicolumn{12}{|c|}{ Car Market Share } \\
\hline $\begin{array}{l}\text { Model } \\
\text { Year }\end{array}$ & $\begin{array}{l}5-10 \\
\mathrm{mpg}\end{array}$ & $\begin{array}{c}10-15 \\
\mathrm{mpg}\end{array}$ & $\begin{array}{c}15-20 \\
\mathrm{mpg}\end{array}$ & $\begin{array}{c}20-25 \\
\mathrm{mpg}\end{array}$ & $\begin{array}{c}25-30 \\
\mathrm{mpg}\end{array}$ & $\begin{array}{c}\text { 30-35 } \\
\text { mpg }\end{array}$ & $\begin{array}{c}35- \\
40 \\
\mathrm{mpg}\end{array}$ & $\begin{array}{c}40- \\
45 \\
\mathrm{mpg}\end{array}$ & $\begin{array}{c}45- \\
50 \\
\mathrm{mpg}\end{array}$ & $\begin{array}{c}50- \\
55 \\
\mathrm{mpg}\end{array}$ & $\begin{array}{c}55- \\
60 \\
\mathrm{mpg}\end{array}$ \\
\hline 1986 & $0.0 \%$ & $0.3 \%$ & $16.3 \%$ & $36.5 \%$ & $36.8 \%$ & $7.3 \%$ & $1.9 \%$ & $0.3 \%$ & $0.3 \%$ & $0.1 \%$ & $0.2 \%$ \\
\hline 1987 & $0.0 \%$ & $0.3 \%$ & $15.3 \%$ & $40.3 \%$ & $33.4 \%$ & $8.7 \%$ & $1.6 \%$ & $0.0 \%$ & $0.3 \%$ & $0.2 \%$ & \\
\hline 1988 & $0.0 \%$ & $0.3 \%$ & $13.8 \%$ & $40.6 \%$ & $34.2 \%$ & $8.2 \%$ & $2.5 \%$ & $0.0 \%$ & $0.2 \%$ & $0.2 \%$ & \\
\hline 1989 & $0.0 \%$ & $0.3 \%$ & $13.8 \%$ & $46.6 \%$ & $30.8 \%$ & $6.2 \%$ & $1.9 \%$ & $0.0 \%$ & $0.3 \%$ & $0.1 \%$ & \\
\hline 1990 & $0.0 \%$ & $0.2 \%$ & $13.4 \%$ & $51.1 \%$ & $28.9 \%$ & $5.3 \%$ & $0.7 \%$ & $0.1 \%$ & $0.3 \%$ & $0.1 \%$ & \\
\hline 1991 & $0.0 \%$ & $0.2 \%$ & $14.5 \%$ & $50.4 \%$ & $27.3 \%$ & $5.9 \%$ & $0.9 \%$ & $0.7 \%$ & $0.1 \%$ & $0.0 \%$ & \\
\hline 1992 & $0.0 \%$ & $0.2 \%$ & $11.8 \%$ & $57.5 \%$ & $22.8 \%$ & $5.1 \%$ & $1.6 \%$ & $0.8 \%$ & $0.1 \%$ & $0.1 \%$ & \\
\hline 1993 & $0.0 \%$ & $0.1 \%$ & $13.1 \%$ & $53.4 \%$ & $25.5 \%$ & $5.5 \%$ & $1.6 \%$ & $0.7 \%$ & $0.0 \%$ & $0.1 \%$ & \\
\hline 1994 & & $0.1 \%$ & $16.1 \%$ & $52.1 \%$ & $24.6 \%$ & $4.9 \%$ & $1.5 \%$ & $0.6 \%$ & $0.0 \%$ & $0.1 \%$ & \\
\hline 1995 & $0.0 \%$ & $0.1 \%$ & $13.5 \%$ & $52.6 \%$ & $25.8 \%$ & $7.1 \%$ & $0.5 \%$ & $0.4 \%$ & $0.0 \%$ & & \\
\hline 1996 & & $0.1 \%$ & $10.4 \%$ & $62.2 \%$ & $21.5 \%$ & $5.1 \%$ & $0.4 \%$ & $0.3 \%$ & & & \\
\hline 1997 & & $0.1 \%$ & $10.9 \%$ & $59.7 \%$ & $25.2 \%$ & $3.7 \%$ & $0.2 \%$ & $0.2 \%$ & & & \\
\hline 1998 & & $0.1 \%$ & $9.9 \%$ & $59.4 \%$ & $26.8 \%$ & $3.4 \%$ & $0.1 \%$ & $0.3 \%$ & & & \\
\hline 1999 & & $0.1 \%$ & $9.9 \%$ & $65.6 \%$ & $22.6 \%$ & $1.6 \%$ & $0.1 \%$ & $0.1 \%$ & & & \\
\hline 2000 & $0.0 \%$ & $0.0 \%$ & $9.2 \%$ & $69.8 \%$ & $19.0 \%$ & $1.6 \%$ & $0.2 \%$ & $0.2 \%$ & & & $0.1 \%$ \\
\hline 2001 & $0.0 \%$ & $0.0 \%$ & $11.8 \%$ & $60.7 \%$ & $19.9 \%$ & $7.0 \%$ & $0.1 \%$ & $0.3 \%$ & $0.0 \%$ & & $0.0 \%$ \\
\hline 2002 & & $0.1 \%$ & $10.1 \%$ & $65.8 \%$ & $17.5 \%$ & $5.9 \%$ & $0.2 \%$ & $0.4 \%$ & $0.0 \%$ & & $0.0 \%$ \\
\hline 2003 & $0.0 \%$ & $0.1 \%$ & $10.4 \%$ & $60.3 \%$ & $24.2 \%$ & $4.1 \%$ & $0.0 \%$ & $0.8 \%$ & $0.0 \%$ & $0.0 \%$ & \\
\hline 2004 & & $0.1 \%$ & $13.1 \%$ & $56.3 \%$ & $27.2 \%$ & $2.2 \%$ & $0.2 \%$ & $0.3 \%$ & $0.6 \%$ & $0.0 \%$ & \\
\hline 2005 & & $0.2 \%$ & $13.5 \%$ & $47.7 \%$ & $30.3 \%$ & $6.4 \%$ & $0.1 \%$ & $0.3 \%$ & $1.5 \%$ & $0.0 \%$ & \\
\hline 2006 & & $0.4 \%$ & $13.8 \%$ & $51.9 \%$ & $23.5 \%$ & $8.9 \%$ & $0.0 \%$ & $0.4 \%$ & $1.1 \%$ & $0.0 \%$ & \\
\hline 2007 & & $0.3 \%$ & $10.1 \%$ & $43.9 \%$ & $32.3 \%$ & $11.1 \%$ & & $0.4 \%$ & $2.0 \%$ & & \\
\hline 2008 & & $0.3 \%$ & $8.9 \%$ & $44.6 \%$ & $33.3 \%$ & $9.9 \%$ & $0.3 \%$ & $0.4 \%$ & $2.3 \%$ & & \\
\hline 2009 & & $0.2 \%$ & $5.1 \%$ & $33.7 \%$ & $40.7 \%$ & $18.1 \%$ & $0.4 \%$ & $0.3 \%$ & $1.5 \%$ & & \\
\hline 2010 & & $0.2 \%$ & $6.1 \%$ & $31.1 \%$ & $37.4 \%$ & $20.2 \%$ & $1.0 \%$ & $1.2 \%$ & $2.8 \%$ & & \\
\hline
\end{tabular}




\begin{tabular}{|c|c|c|c|c|c|c|c|c|c|c|c|}
\hline \multicolumn{12}{|c|}{ Light Truck Market Share } \\
\hline $\begin{array}{c}\text { Model } \\
\text { Year }\end{array}$ & $\begin{array}{l}\text { 5-10 } \\
\mathrm{mpg}\end{array}$ & $\begin{array}{c}10-15 \\
\mathrm{mpg}\end{array}$ & $\begin{array}{c}15-20 \\
\mathrm{mpg}\end{array}$ & $\begin{array}{c}20-25 \\
\mathrm{mpg}\end{array}$ & $\begin{array}{c}25- \\
30 \\
\mathrm{mpg}\end{array}$ & $\begin{array}{c}30- \\
35 \\
\mathrm{mpg}\end{array}$ & $\begin{array}{c}35- \\
40 \\
\mathrm{mpg}\end{array}$ & $\begin{array}{c}40- \\
45 \\
\mathrm{mpg}\end{array}$ & $\begin{array}{c}45- \\
50 \\
\mathrm{mpg}\end{array}$ & $\begin{array}{c}50- \\
55 \\
\mathrm{mpg}\end{array}$ & $\begin{array}{c}55- \\
60 \\
\mathrm{mpg}\end{array}$ \\
\hline 1975 & $27.6 \%$ & $52.1 \%$ & $17.9 \%$ & $2.5 \%$ & & & & & & & \\
\hline 1976 & $12.3 \%$ & $66.0 \%$ & $16.6 \%$ & $5.1 \%$ & & & & & & & \\
\hline 1977 & $3.8 \%$ & $66.0 \%$ & $19.8 \%$ & $9.1 \%$ & $1.3 \%$ & & & & & & \\
\hline 1978 & $3.1 \%$ & $68.6 \%$ & $18.9 \%$ & $8.5 \%$ & $0.9 \%$ & & & & & & \\
\hline 1979 & $14.4 \%$ & $59.1 \%$ & $18.6 \%$ & $6.7 \%$ & $1.2 \%$ & & & & & & \\
\hline 1980 & $0.2 \%$ & $40.7 \%$ & $37.9 \%$ & $19.1 \%$ & $1.5 \%$ & & $0.6 \%$ & & & & \\
\hline 1981 & $0.0 \%$ & $22.8 \%$ & $50.7 \%$ & $16.1 \%$ & $7.1 \%$ & $2.6 \%$ & $0.7 \%$ & & & & \\
\hline 1982 & & $21.8 \%$ & $45.3 \%$ & $23.7 \%$ & $7.9 \%$ & $0.7 \%$ & $0.6 \%$ & & & & \\
\hline 1983 & & $20.8 \%$ & $41.4 \%$ & $27.1 \%$ & $9.7 \%$ & $0.9 \%$ & $0.2 \%$ & & & & \\
\hline 1984 & $0.1 \%$ & $23.9 \%$ & $42.8 \%$ & $25.4 \%$ & $7.0 \%$ & $0.7 \%$ & $0.1 \%$ & & & & \\
\hline 1985 & $0.0 \%$ & $27.2 \%$ & $40.2 \%$ & $27.7 \%$ & $4.7 \%$ & $0.3 \%$ & $0.0 \%$ & & & & \\
\hline 1986 & & $19.4 \%$ & $42.0 \%$ & $33.2 \%$ & $5.3 \%$ & $0.1 \%$ & $0.0 \%$ & & & & \\
\hline 1987 & & $16.9 \%$ & $44.2 \%$ & $32.9 \%$ & $6.0 \%$ & $0.0 \%$ & $0.0 \%$ & & & & \\
\hline 1988 & $0.0 \%$ & $17.4 \%$ & $53.6 \%$ & $22.2 \%$ & $6.8 \%$ & & & & & & \\
\hline 1989 & & $18.3 \%$ & $54.9 \%$ & $24.2 \%$ & $2.6 \%$ & & & & & & \\
\hline 1990 & $0.3 \%$ & $21.4 \%$ & $56.2 \%$ & $18.5 \%$ & $3.5 \%$ & & & & & & \\
\hline 1991 & & $15.7 \%$ & $61.8 \%$ & $20.5 \%$ & $2.1 \%$ & & & & & & \\
\hline 1992 & & $18.2 \%$ & $61.7 \%$ & $20.0 \%$ & $0.1 \%$ & & & & & & \\
\hline 1993 & & $13.1 \%$ & $69.1 \%$ & $17.8 \%$ & $0.0 \%$ & & & & & & \\
\hline 1994 & & $21.7 \%$ & $62.3 \%$ & $15.8 \%$ & $0.1 \%$ & & & & & & \\
\hline 1995 & & $23.5 \%$ & $60.8 \%$ & $15.6 \%$ & $0.0 \%$ & & & & & & \\
\hline 1996 & & $21.0 \%$ & $61.9 \%$ & $17.0 \%$ & $0.1 \%$ & & & & & & \\
\hline 1997 & & $18.3 \%$ & $69.8 \%$ & $11.9 \%$ & $0.0 \%$ & & & & & & \\
\hline 1998 & & $21.8 \%$ & $63.4 \%$ & $14.8 \%$ & $0.0 \%$ & & & & & & \\
\hline 1999 & & $22.4 \%$ & $65.1 \%$ & $12.4 \%$ & & & & & & & \\
\hline 2000 & & $15.7 \%$ & $73.4 \%$ & $10.9 \%$ & & & & & & & \\
\hline
\end{tabular}




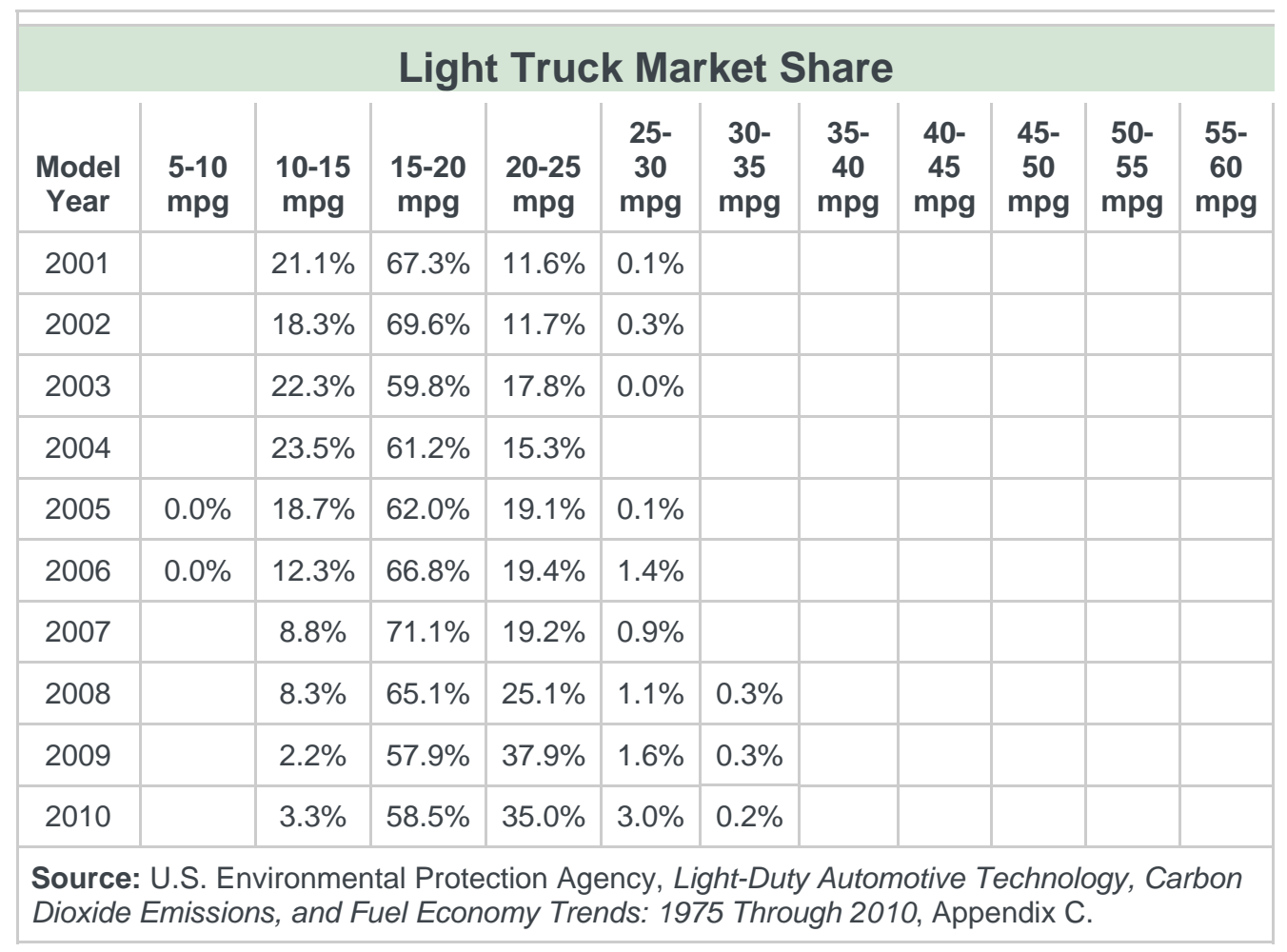




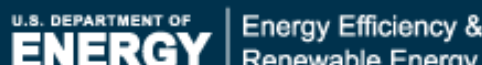 \\ Renewable Energy}

\section{Vehicle Technologies Program}

Fact \#693: September 19, 2011 Average Vehicle Footprint for Cars and Light Trucks

A vehicle footprint is the area defined by the four points where the tires touch the ground. It is calculated as the product of the wheelbase and the average track width of the vehicle. The upcoming Corporate Average Fuel Economy (CAFE) Standards have fuel economy targets based on the vehicle footprint. The average footprint for all cars sold in model year (MY) 2010 was 45.2 square feet (sq ft), down just $0.2 \mathrm{sq} f \mathrm{ft}$ from 2008. The average footprint for light trucks was higher - 54.0 in 2010.

Average Vehicle Footprint, 2008-2010

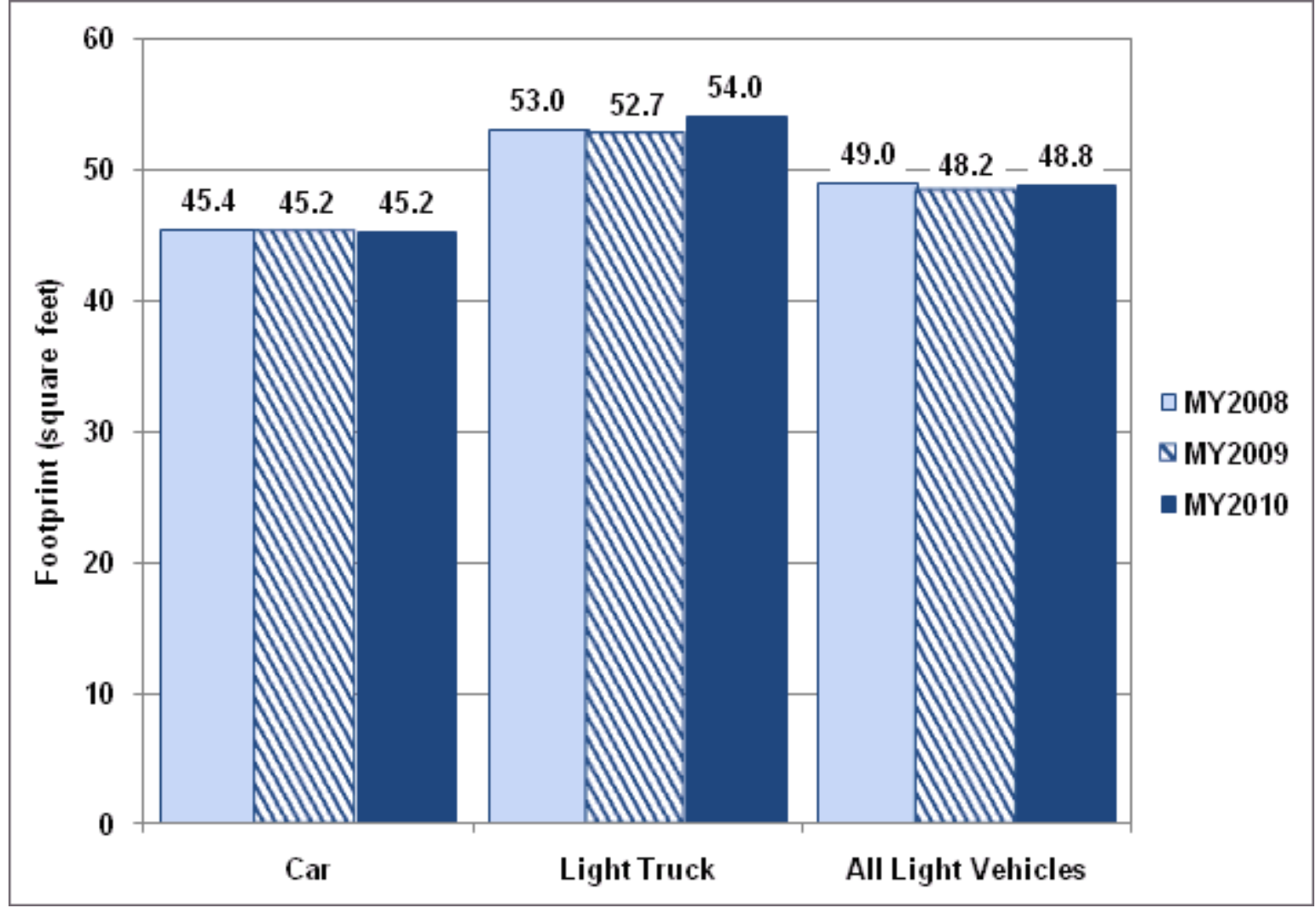




\section{Supporting Information}

\begin{tabular}{|c|c|c|c|}
\hline \multicolumn{4}{|c|}{ Average Vehicle Footprint, 2008-2010 } \\
\hline Model Year & Car & Light Truck & All Light Vehicles \\
\hline 2008 & 45.4 & 53.0 & 49.0 \\
\hline 2009 & 45.2 & 52.7 & 48.2 \\
\hline 2010 & 45.2 & 54.0 & 48.8 \\
\hline $\begin{array}{l}\text { Source: U.S. Environmental Protection Agency, Light-Duty } \\
\text { Automotive Technology, Carbon Dioxide Emissions, and Fuel } \\
\text { Economy Trends: 1975 Through 2010, Table 2. }\end{array}$ \\
\hline
\end{tabular}




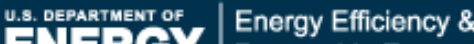 \\ Renewable Energy}

\section{Vehicle Technologies Program}

\section{Fact \#694: September 26, 2011 Costs of Owning a Vehicle by State}

There are many costs involved in owning a vehicle, such as depreciation, interest on financing, taxes and fees, insurance premiums, fuel, maintenance, and repairs.

Research has been done to calculate the difference in those costs over a five-year period on a State by State basis. The difference can be as much as $\$ 10,000$. South Dakota, New Hampshire, and Wisconsin are the least expensive States in terms of vehicle ownership, while Hawaii, Alaska, and California are the most expensive. 
Total Cost to Own a Vehicle over a Five-Year Period*

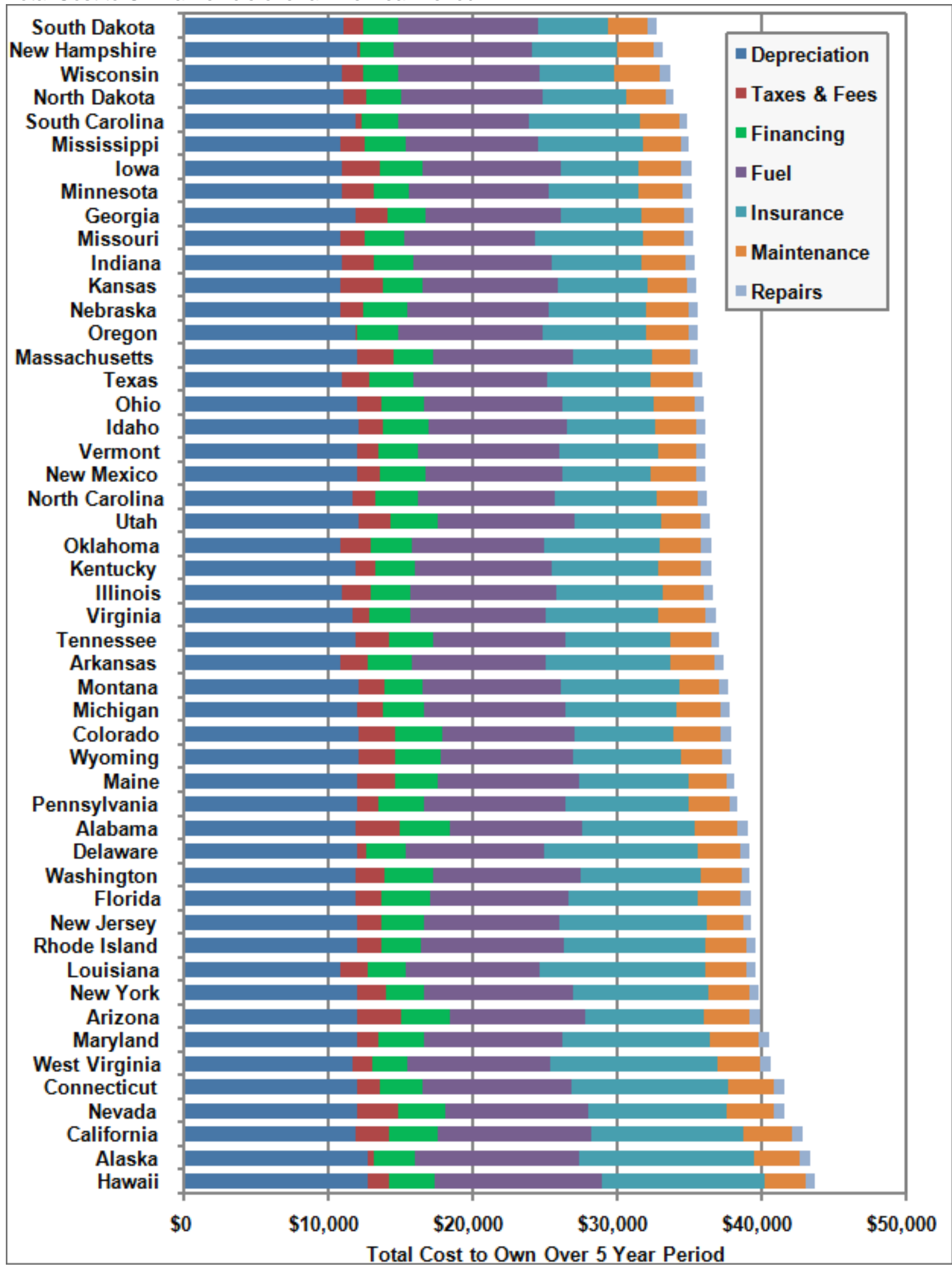

* Based on a 2011 Toyota Camry 4-door sedan on August 3, 2011. Different vehicle models will have different costs. 


\section{Supporting Information}

\begin{tabular}{|c|c|c|c|c|c|c|c|c|}
\hline \multicolumn{9}{|c|}{ Total Cost to Own a Vehicle over a Five-Year Period* } \\
\hline State & Depreciation & $\begin{array}{l}\text { Taxes \& } \\
\text { Fees }\end{array}$ & Financing & Fuel & Insurance & Maintenance & Repairs & $\begin{array}{l}\text { True Cost } \\
\text { to Own }\end{array}$ \\
\hline Alabama & $\$ 11,860$ & $\$ 3,082$ & $\$ 3,403$ & $\$ 9,252$ & $\$ 7,754$ & $\$ 2,977$ & $\$ 665$ & $\$ 38,993$ \\
\hline Alaska & $\$ 12,666$ & $\$ 489$ & $\$ 2,856$ & $\$ 11,365$ & $\$ 12,135$ & $\$ 3,137$ & $\$ 744$ & $\$ 43,392$ \\
\hline Arizona & $\$ 11,922$ & $\$ 3,150$ & $\$ 3,325$ & $\$ 9,405$ & $\$ 8,167$ & $\$ 3,198$ & $\$ 732$ & $\$ 39,899$ \\
\hline Arkansas & $\$ 10,853$ & $\$ 1,821$ & $\$ 3,107$ & $\$ 9,222$ & $\$ 8,687$ & $\$ 3,035$ & $\$ 683$ & $\$ 37,408$ \\
\hline California & $\$ 11,842$ & $\$ 2,355$ & $\$ 3,371$ & $\$ 10,660$ & $\$ 10,500$ & $\$ 3,324$ & $\$ 773$ & $\$ 42,825$ \\
\hline Colorado & $\$ 12,072$ & $\$ 2,560$ & $\$ 3,217$ & $\$ 9,222$ & $\$ 6,843$ & $\$ 3,204$ & $\$ 744$ & $\$ 37,862$ \\
\hline Connecticut & $\$ 11,927$ & $\$ 1,629$ & $\$ 2,989$ & $\$ 10,263$ & $\$ 10,833$ & $\$ 3,151$ & $\$ 726$ & $\$ 41,518$ \\
\hline Delaware & $\$ 11,927$ & $\$ 708$ & $\$ 2,731$ & $\$ 9,557$ & $\$ 10,693$ & $\$ 2,889$ & $\$ 671$ & $\$ 39,176$ \\
\hline Florida & $\$ 11,860$ & $\$ 1,753$ & $\$ 3,366$ & $\$ 9,650$ & $\$ 8,977$ & $\$ 2,960$ & $\$ 665$ & $\$ 39,231$ \\
\hline Georgia & $\$ 11,860$ & $\$ 2,251$ & $\$ 2,651$ & $\$ 9,344$ & $\$ 5,610$ & $\$ 2,850$ & $\$ 631$ & $\$ 35,197$ \\
\hline Hawaii & $\$ 12,666$ & $\$ 1,468$ & $\$ 3,172$ & $\$ 11,579$ & $\$ 11,299$ & $\$ 2,868$ & $\$ 648$ & $\$ 43,700$ \\
\hline Idaho & $\$ 12,072$ & $\$ 1,723$ & $\$ 3,096$ & $\$ 9,650$ & $\$ 6,055$ & $\$ 2,836$ & $\$ 631$ & $\$ 36,063$ \\
\hline Illinois & $\$ 10,969$ & $\$ 1,973$ & $\$ 2,739$ & $\$ 10,109$ & $\$ 7,326$ & $\$ 2,868$ & $\$ 637$ & $\$ 36,621$ \\
\hline Indiana & $\$ 10,969$ & $\$ 2,196$ & $\$ 2,655$ & $\$ 9,679$ & $\$ 6,187$ & $\$ 2,985$ & $\$ 671$ & $\$ 35,342$ \\
\hline lowa & $\$ 10,969$ & $\$ 2,626$ & $\$ 2,903$ & $\$ 9,557$ & $\$ 5,363$ & $\$ 3,002$ & $\$ 677$ & $\$ 35,097$ \\
\hline Kansas & $\$ 10,853$ & $\$ 2,882$ & $\$ 2,758$ & $\$ 9,405$ & $\$ 6,182$ & $\$ 2,763$ & $\$ 601$ & $\$ 35,444$ \\
\hline Kentucky & $\$ 11,853$ & $\$ 1,370$ & $\$ 2,753$ & $\$ 9,527$ & $\$ 7,352$ & $\$ 2,970$ & $\$ 671$ & $\$ 36,496$ \\
\hline Louisiana & $\$ 10,860$ & $\$ 1,835$ & $\$ 2,622$ & $\$ 9,252$ & $\$ 11,561$ & $\$ 2,847$ & $\$ 625$ & $\$ 39,602$ \\
\hline Maine & $\$ 11,927$ & $\$ 2,649$ & $\$ 2,991$ & $\$ 9,834$ & $\$ 7,497$ & $\$ 2,645$ & $\$ 577$ & $\$ 38,120$ \\
\hline Maryland & $\$ 11,927$ & $\$ 1,544$ & $\$ 3,097$ & $\$ 9,588$ & $\$ 10,231$ & $\$ 3,375$ & $\$ 797$ & $\$ 40,559$ \\
\hline Massachusetts & $\$ 11,927$ & $\$ 2,565$ & $\$ 2,735$ & $\$ 9,650$ & $\$ 5,551$ & $\$ 2,604$ & $\$ 560$ & $\$ 35,592$ \\
\hline Michigan & $\$ 11,969$ & $\$ 1,825$ & $\$ 2,785$ & $\$ 9,864$ & $\$ 7,674$ & $\$ 3,009$ & $\$ 683$ & $\$ 37,809$ \\
\hline Minnesota & $\$ 10,969$ & $\$ 2,202$ & $\$ 2,399$ & $\$ 9,650$ & $\$ 6,248$ & $\$ 3,024$ & $\$ 683$ & $\$ 35,175$ \\
\hline Mississippi & $\$ 10,860$ & $\$ 1,665$ & $\$ 2,770$ & $\$ 9,190$ & $\$ 7,260$ & $\$ 2,636$ & $\$ 565$ & $\$ 34,946$ \\
\hline Missouri & $\$ 10,853$ & $\$ 1,650$ & $\$ 2,699$ & $\$ 9,099$ & $\$ 7,449$ & $\$ 2,864$ & $\$ 637$ & $\$ 35,251$ \\
\hline Montana & $\$ 12,072$ & $\$ 1,841$ & $\$ 2,626$ & $\$ 9,557$ & $\$ 8,221$ & $\$ 2,695$ & $\$ 612$ & $\$ 37,624$ \\
\hline Nebraska & $\$ 10,853$ & $\$ 1,498$ & $\$ 3,127$ & $\$ 9,803$ & $\$ 6,688$ & $\$ 2,928$ & $\$ 659$ & $\$ 35,556$ \\
\hline Nevada & $\$ 11,922$ & $\$ 2,873$ & $\$ 3,244$ & $\$ 9,925$ & $\$ 9,610$ & $\$ 3,223$ & $\$ 744$ & $\$ 41,541$ \\
\hline $\begin{array}{l}\text { New } \\
\text { Hampshire }\end{array}$ & $\$ 11,927$ & $\$ 220$ & $\$ 2,369$ & $\$ 9,527$ & $\$ 5,921$ & $\$ 2,559$ & $\$ 571$ & $\$ 33,094$ \\
\hline New Jersey & $\$ 11,927$ & $\$ 1,746$ & $\$ 2,947$ & $\$ 9,313$ & $\$ 10,248$ & $\$ 2,557$ & $\$ 543$ & $\$ 39,281$ \\
\hline
\end{tabular}




\begin{tabular}{|c|c|c|c|c|c|c|c|c|}
\hline \multicolumn{9}{|c|}{ Total Cost to Own a Vehicle over a Five-Year Period* } \\
\hline State & Depreciation & $\begin{array}{c}\text { Taxes \& } \\
\text { Fees }\end{array}$ & Financing & Fuel & Insurance & Maintenance & Repairs & $\begin{array}{c}\text { True Cost } \\
\text { to Own }\end{array}$ \\
\hline New Mexico & $\$ 11,922$ & $\$ 1,673$ & $\$ 3,145$ & $\$ 9,466$ & $\$ 6,086$ & $\$ 3,122$ & $\$ 715$ & $\$ 36,129$ \\
\hline New York & $\$ 11,942$ & $\$ 1,992$ & $\$ 2,672$ & $\$ 10,293$ & $\$ 9,443$ & $\$ 2,826$ & $\$ 618$ & $\$ 39,786$ \\
\hline North Carolina & $\$ 11,639$ & $\$ 1,588$ & $\$ 2,949$ & $\$ 9,466$ & $\$ 7,042$ & $\$ 2,905$ & $\$ 648$ & $\$ 36,237$ \\
\hline North Dakota & $\$ 11,072$ & $\$ 1,576$ & $\$ 2,373$ & $\$ 9,834$ & $\$ 5,732$ & $\$ 2,730$ & $\$ 601$ & $\$ 33,918$ \\
\hline Ohio & $\$ 11,969$ & $\$ 1,684$ & $\$ 2,952$ & $\$ 9,619$ & $\$ 6,317$ & $\$ 2,848$ & $\$ 631$ & $\$ 36,020$ \\
\hline Oklahoma & $\$ 10,853$ & $\$ 2,049$ & $\$ 2,811$ & $\$ 9,252$ & $\$ 7,921$ & $\$ 2,937$ & $\$ 654$ & $\$ 36,477$ \\
\hline Oregon & $\$ 11,842$ & $\$ 129$ & $\$ 2,854$ & $\$ 10,048$ & $\$ 7,121$ & $\$ 2,908$ & $\$ 677$ & $\$ 35,579$ \\
\hline Pennsylvania & $\$ 11,927$ & $\$ 1,516$ & $\$ 3,170$ & $\$ 9,742$ & $\$ 8,580$ & $\$ 2,797$ & $\$ 618$ & $\$ 38,350$ \\
\hline Rhode Island & $\$ 11,927$ & $\$ 1,714$ & $\$ 2,777$ & $\$ 9,864$ & $\$ 9,776$ & $\$ 2,850$ & $\$ 631$ & $\$ 39,539$ \\
\hline South Carolina & $\$ 11,860$ & $\$ 377$ & $\$ 2,534$ & $\$ 9,099$ & $\$ 7,700$ & $\$ 2,715$ & $\$ 589$ & $\$ 34,874$ \\
\hline South Dakota & $\$ 11,072$ & $\$ 1,325$ & $\$ 2,426$ & $\$ 9,679$ & $\$ 4,815$ & $\$ 2,823$ & $\$ 631$ & $\$ 32,771$ \\
\hline Tennessee & $\$ 11,853$ & $\$ 2,348$ & $\$ 3,002$ & $\$ 9,190$ & $\$ 7,244$ & $\$ 2,825$ & $\$ 612$ & $\$ 37,074$ \\
\hline Texas & $\$ 10,922$ & $\$ 1,854$ & $\$ 3,092$ & $\$ 9,222$ & $\$ 7,159$ & $\$ 2,991$ & $\$ 671$ & $\$ 35,911$ \\
\hline Utah & $\$ 12,072$ & $\$ 2,243$ & $\$ 3,247$ & $\$ 9,466$ & $\$ 5,978$ & $\$ 2,784$ & $\$ 612$ & $\$ 36,402$ \\
\hline Vermont & $\$ 11,927$ & $\$ 1,525$ & $\$ 2,775$ & $\$ 9,803$ & $\$ 6,816$ & $\$ 2,660$ & $\$ 577$ & $\$ 36,083$ \\
\hline Virginia & $\$ 11,639$ & $\$ 1,208$ & $\$ 2,838$ & $\$ 9,313$ & $\$ 7,873$ & $\$ 3,186$ & $\$ 744$ & $\$ 36,801$ \\
\hline Washington & $\$ 11,842$ & $\$ 2,059$ & $\$ 3,313$ & $\$ 10,263$ & $\$ 8,344$ & $\$ 2,771$ & $\$ 601$ & $\$ 39,193$ \\
\hline West Virginia & $\$ 11,639$ & $\$ 1,426$ & $\$ 2,365$ & $\$ 9,896$ & $\$ 11,615$ & $\$ 2,970$ & $\$ 671$ & $\$ 40,582$ \\
\hline Wisconsin & $\$ 10,969$ & $\$ 1,427$ & $\$ 2,424$ & $\$ 9,742$ & $\$ 5,249$ & $\$ 3,156$ & $\$ 732$ & $\$ 33,699$ \\
\hline Wyoming & $\$ 12,072$ & $\$ 2,540$ & $\$ 3,138$ & $\$ 9,222$ & $\$ 7,444$ & $\$ 2,826$ & $\$ 631$ & $\$ 37,873$ \\
\hline
\end{tabular}




\section{u.s. Department of | Energy Efficiency \& \\ Renewable Energy}

\section{Vehicle Technologies Program}

Fact \#695: October 3, 2011

New Car Dealerships

The number of new car dealerships has declined by nearly 25\% from 1991 to 2011 . The largest decline in this period was for dealerships that sold 150-399 new cars annually. The smallest dealerships, selling less than 150 new cars annually, declined from 1991 to 2011 , but grew in number from 2001 to 2011 . The number of large dealers (750 new car sales and up) was the highest in 2001.

Number of New Car Dealerships by Sales Volume

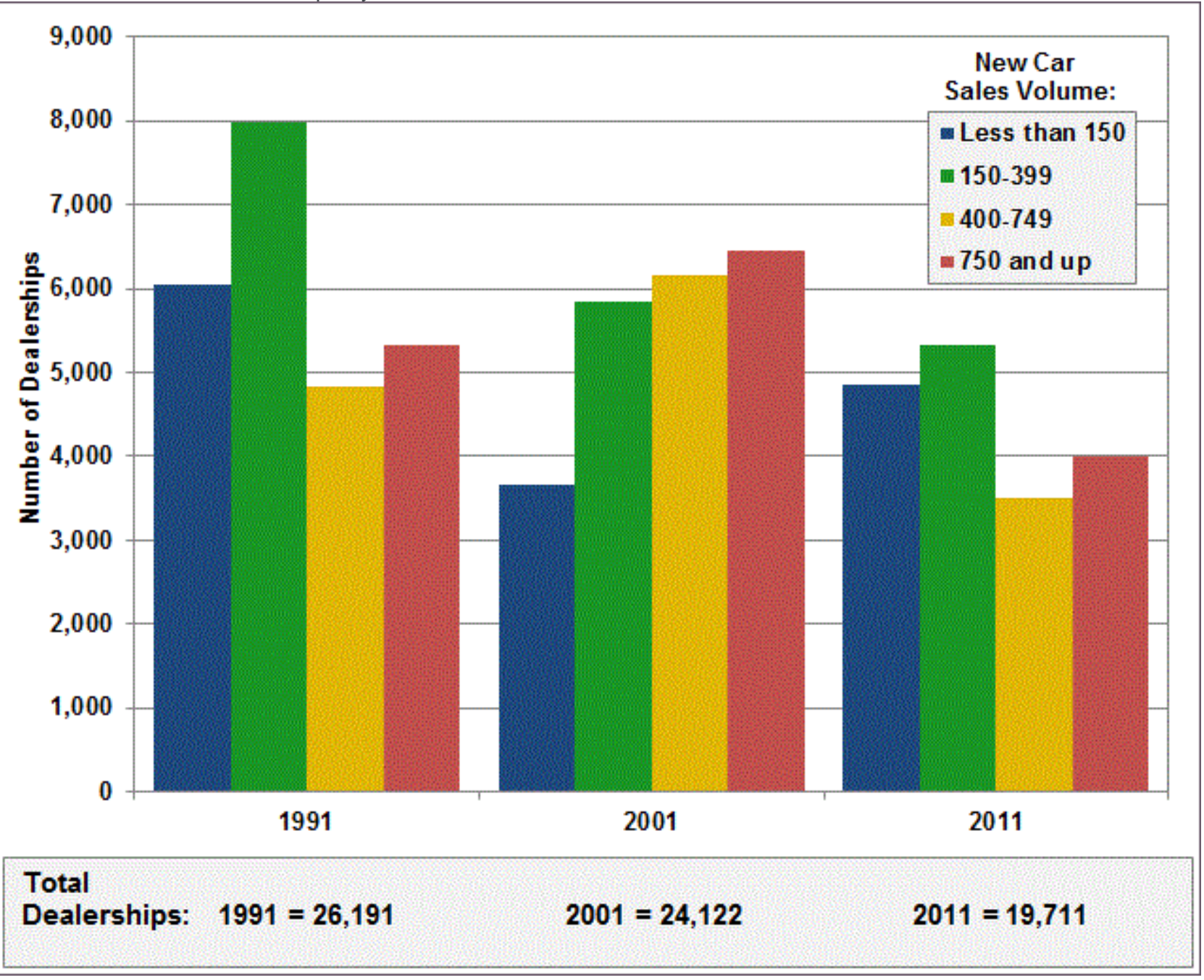




\section{Supporting Information}

\begin{tabular}{|c|c|c|c|c|c|}
\hline \multicolumn{6}{|c|}{ Number of New Car Dealerships by Sales Volume } \\
\hline $\begin{array}{c}\text { New } \\
\text { Car } \\
\text { Sales } \\
\text { Volume }\end{array}$ & 1991 & 2001 & 2011 & $\begin{array}{c}\text { Percent } \\
\text { change } \\
\text { from } \\
2011 \text { to } \\
1991\end{array}$ & $\begin{array}{c}\text { Percent } \\
\text { change } \\
\text { from } \\
2011 \text { to } \\
2001\end{array}$ \\
\hline $\begin{array}{l}\text { Less } \\
\text { than } \\
150\end{array}$ & 6,050 & 3,669 & 4,864 & $-19.6 \%$ & $32.6 \%$ \\
\hline 150-399 & 7,986 & 5,848 & 5,337 & $-33.2 \%$ & $-8.7 \%$ \\
\hline $400-749$ & 4,840 & 6,158 & 3,512 & $-27.4 \%$ & $-43.0 \%$ \\
\hline $\begin{array}{l}750 \text { and } \\
\text { up }\end{array}$ & 5,324 & 6,446 & 3,987 & $-25.1 \%$ & $-38.1 \%$ \\
\hline Total & 26,191 & 24,122 & 19,711 & $-24.7 \%$ & $-18.3 \%$ \\
\hline \multicolumn{6}{|c|}{ Source: NADA DATA 2011.} \\
\hline
\end{tabular}




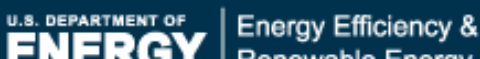 \\ Renewable Energy}

\section{Vehicle Technologies Program}

\section{Fact \#696: October 10, 2011 Top Ten "Real World" Fuel Economy Leaders}

The Environmental Protection Agency (EPA) fuel economy ratings on the window stickers of new cars are based on strict test cycles conducted in a controlled laboratory setting. These official EPA estimates do not reflect all the varied conditions encountered in real world driving such as congestion, terrain, weather, driving style, fuel blends and other factors. On the website www.fueleconomy.gov, drivers are encouraged to enter their real world fuel economy to compare their results with EPA ratings and with other car owners across the nation. The model year (MY) 2004 - 2006 Honda Insight is currently the vehicle in the database with the best real world fuel economy, followed by the MY 1990 - 1994 Geo Metro. Both vehicles have real world average fuel economies higher than the EPA ratings. The table also shows the number of users who have entered data for that particular vehicle type - the MY 2010-2011 Toyota Prius (\#4 on the list) has 158 people who entered their fuel economy information.

If you would like to enter fuel economy information for your vehicle, go to Your MPG page on the www.fueleconomy.gov website.

\section{Top Ten "Real World" Fuel Economy Leaders}

1.

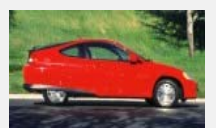

Honda Insight Model Years 2004-2006

3 cyl, 1.0L, Manual 5-spd, Regular

2.

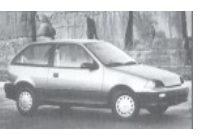

3.

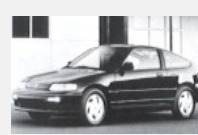

Geo Metro XFI Model Years 1990-1994

3 cyl, 1.0L, Manual 5-spd, Regular

Honda Civic CRX HF Model Years 1990-1991

4 cyl, 1.5L, Manual 5-spd, Regular
User Average 71.2

(based on 12 Your MPG users)

EPA Combined 52

User Average $\mathbf{5 0 . 4}$

(based on 15 Your MPG users)

EPA Combined 47

User Average $\mathbf{5 0 . 2}$

(based on 11 Your MPG users)

EPA Combined 43 


\begin{tabular}{|c|c|c|c|}
\hline \multicolumn{4}{|c|}{ Top Ten "Real World" Fuel Economy Leaders } \\
\hline \multirow[t]{2}{*}{4.} & \multirow{2}{*}{ (2) } & Toyota Prius Model Years 2010-2011 & $\begin{array}{l}\text { User Average } 49.1 \\
\text { (based on } 158 \text { Your MPG } \\
\text { users) }\end{array}$ \\
\hline & & $\begin{array}{l}4 \text { cyl, 1.8L, Automatic (variable gear ratios), } \\
\text { Regular }\end{array}$ & EPA Combined 50 \\
\hline \multirow[t]{2}{*}{5.} & \multirow[t]{2}{*}{$=0$} & Honda Insight Model Years 2010-2011 & $\begin{array}{l}\text { User Average } 48.6 \\
\text { (based on } 18 \text { Your MPG } \\
\text { users) }\end{array}$ \\
\hline & & 4 cyl, 1.3L, Auto(AV-S7), Regular & EPA Combined 41 \\
\hline \multirow[t]{2}{*}{6.} & & $\begin{array}{l}\text { Volkswagen Jetta Wagon Model Years } \\
\text { 2002-2003 }\end{array}$ & $\begin{array}{l}\text { User Average } 48.1 \\
\text { (based on } 23 \text { Your MPG } \\
\text { users) }\end{array}$ \\
\hline & & 4 cyl, 1.9L, Manual 5-spd, Diesel & EPA Combined 39 \\
\hline \multirow[t]{2}{*}{7.} & \multirow[t]{2}{*}{$=0$} & Honda Civic Hybrid Model Years 2003-2005 & $\begin{array}{l}\text { User Average } 47.9 \\
\text { (based on } 20 \text { Your MPG } \\
\text { users) }\end{array}$ \\
\hline & & 4 cyl, 1.3L, Manual 5-spd, Regular & EPA Combined 41 \\
\hline \multirow[t]{2}{*}{8.} & & Volkswagen Golf Model Years 2000-2003 & $\begin{array}{l}\text { User Average } 47.1 \\
\text { (based on } 52 \text { Your MPG } \\
\text { users) }\end{array}$ \\
\hline & & 4 cyl, 1.9L, Manual 5-spd, Diesel & EPA Combined 38 \\
\hline \multirow[t]{2}{*}{9.} & \multirow[t]{2}{*}{$50=0$} & Honda Civic HB VX Model Years 1992-1995 & $\begin{array}{l}\text { User Average } 47.1 \\
\text { (based on } 14 \text { Your MPG } \\
\text { users) }\end{array}$ \\
\hline & & 4 cyl, 1.5L, Manual 5-spd, Regular & EPA Combined 43 \\
\hline \multirow[t]{2}{*}{10.} & \multirow[t]{2}{*}{ 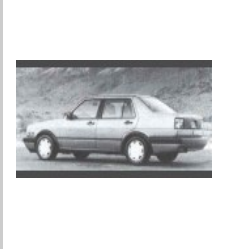 } & Volkswagen Jetta Model Years 1985-1991 & $\begin{array}{l}\text { User Average } 46.4 \\
\text { (based on } 13 \text { Your MPG } \\
\text { users) }\end{array}$ \\
\hline & & 4 cyl, 1.6L, Manual 5-spd, Diesel & EPA Combined 34 \\
\hline
\end{tabular}




\section{Vehicle Technologies Program}

\section{Fact \#697: October 17, 2011 \\ Comparison of Vehicles per Thousand People in Selected Countries/Regions}

The U S. data for vehicles per thousand people are displayed in the line which goes from 1900 to 2009. The points labeled on that line show data for other countries/regions around the world and how their vehicles per thousand people compare to the U.S. at two different points in time, 1999 and 2009. For instance, the graph shows that in 1999 Western Europe's vehicles per thousand people was about where the U.S. was in 1969, but by 2009 it was about where the U.S. was in 1972. The lower part of the graph is enlarged below, showing that China moved from where the U.S. was in 1912 to where the U.S. was in 1917.

Vehicles per Thousand People: US (Over Time) Compared to Other Countries/Regions

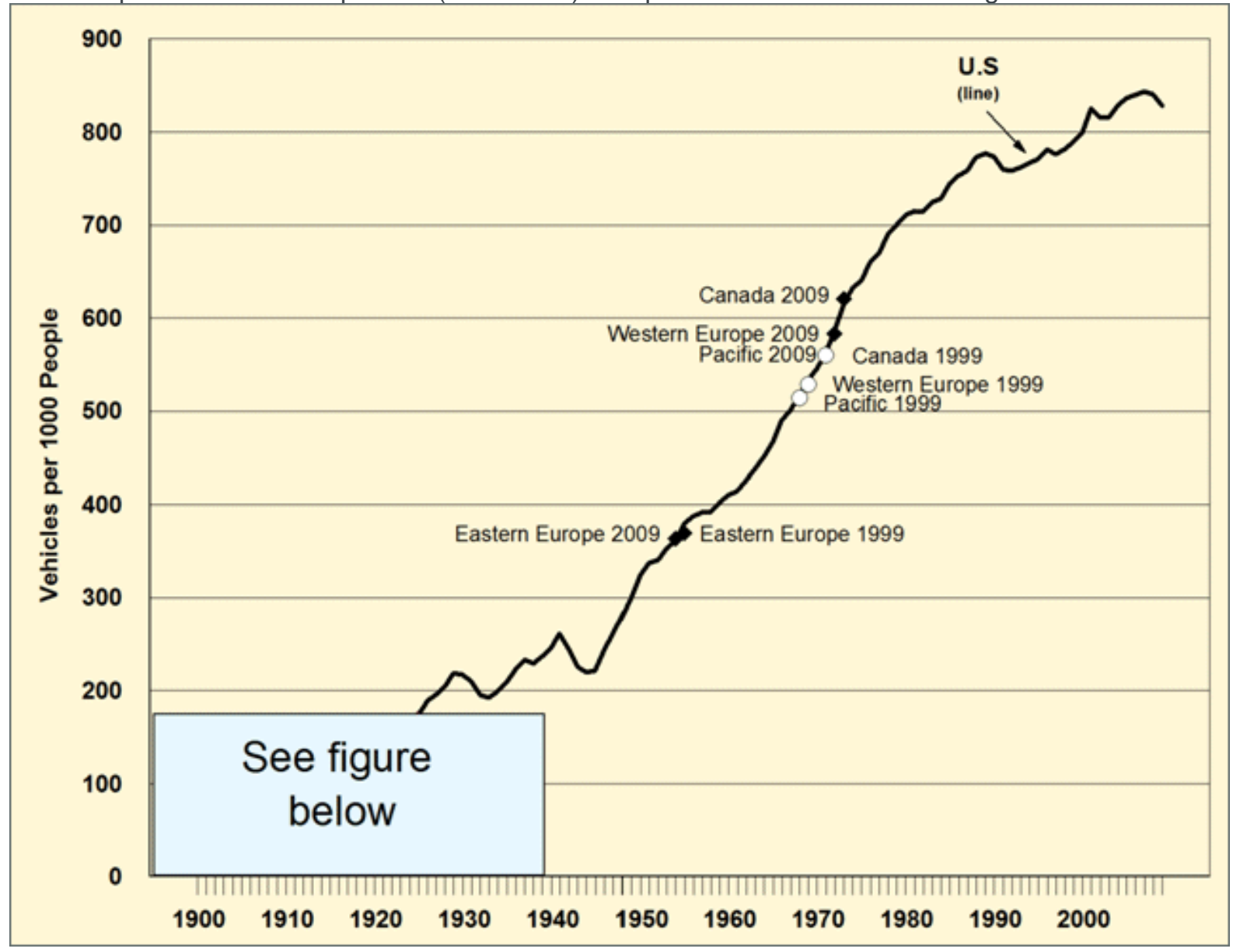


Enlarged bottom left of graph

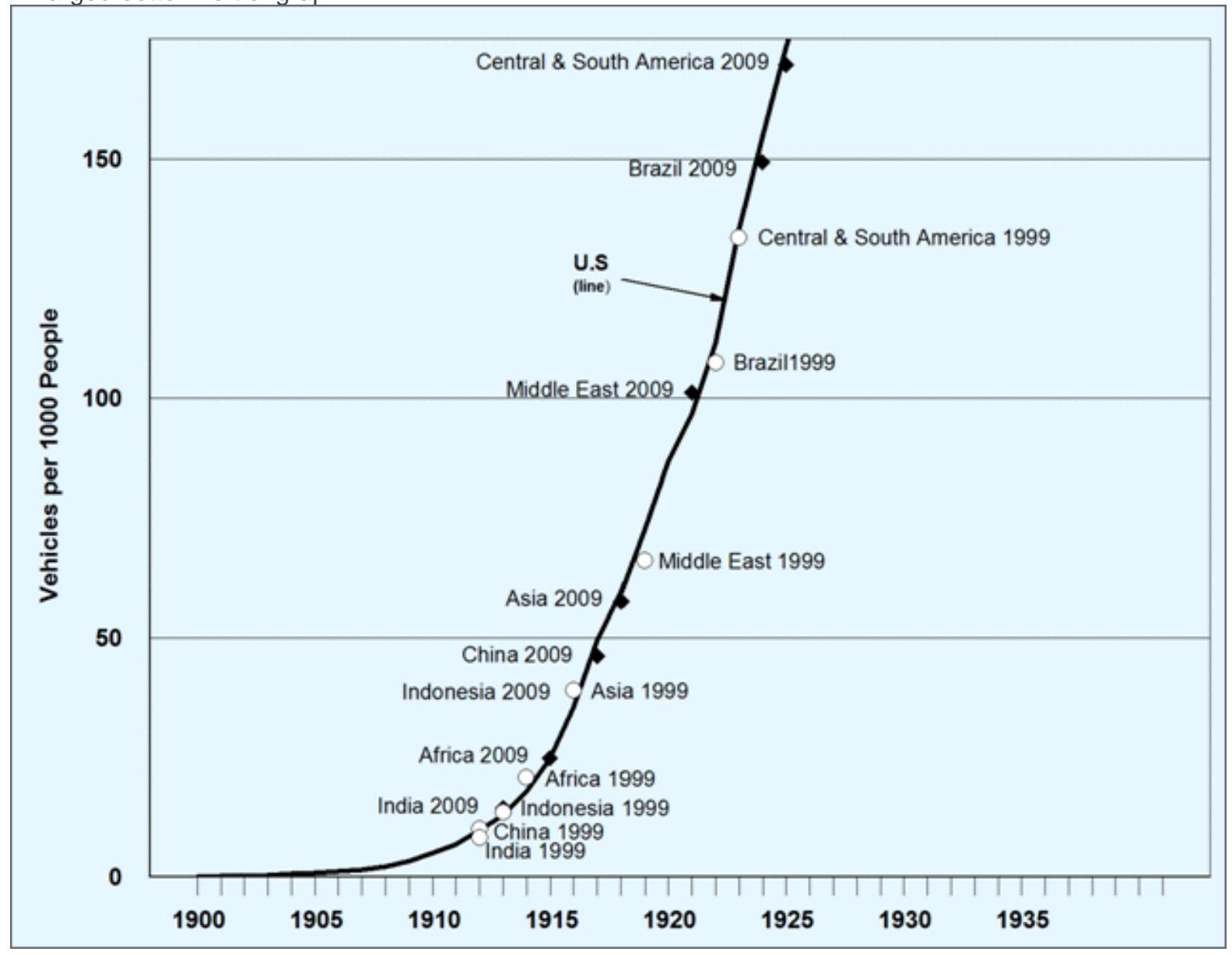




\section{Supporting Information}

\begin{tabular}{|c|c|c|}
\hline \multicolumn{3}{|c|}{$\begin{array}{l}\text { Vehicles per Thousand People in Other } \\
\text { Countries, } 1999 \text { and } 2009\end{array}$} \\
\hline \multirow[b]{2}{*}{ Country/Region } & \multicolumn{2}{|c|}{$\begin{array}{l}\text { Vehicles per } \\
1,000 \text { people }\end{array}$} \\
\hline & 1999 & 2009 \\
\hline Africa & 20.9 & 24.9 \\
\hline Asia, Far East & 39.1 & 157.7 \\
\hline Asia, Middle East & 66.2 & 101.2 \\
\hline Brazil & 107.5 & 149.2 \\
\hline Canada & 560 & 620.9 \\
\hline Central \& South America & 133.6 & 169.7 \\
\hline China & 10.2 & 46.2 \\
\hline Europe, East & 370 & 363.9 \\
\hline Europe, West & 528.8 & 583.3 \\
\hline India & 8.3 & 14.4 \\
\hline Indonesia & 13.7 & 35.9 \\
\hline Pacific & 513.9 & 560.9 \\
\hline $\begin{array}{l}\text { Source: Oak Ridge Nation } \\
\text { Transportation Energy Data } \\
\text { ORNL-6986, June } 2011 .\end{array}$ & $\begin{array}{l}\text { aborator } \\
\text { k: Editic }\end{array}$ & \\
\hline
\end{tabular}




\begin{tabular}{|c|c|c|c|c|c|c|c|c|c|}
\hline \multicolumn{10}{|c|}{ Vehicles per Thousand People in the United States, 1990 and 2009} \\
\hline Year & $\begin{array}{c}\text { U.S. } \\
\text { vehicles } \\
\text { per } \\
1,000 \\
\text { people }\end{array}$ & Year & $\begin{array}{c}\text { U.S. } \\
\text { vehicles } \\
\text { per } \\
1,000 \\
\text { people }\end{array}$ & Year & $\begin{array}{c}\text { U.S. } \\
\text { vehicles } \\
\text { per } \\
1,000 \\
\text { people }\end{array}$ & Year & $\begin{array}{c}\text { U.S. } \\
\text { vehicles } \\
\text { per } \\
1,000 \\
\text { people }\end{array}$ & Year & $\begin{array}{c}\text { U.S. } \\
\text { vehicles } \\
\text { per } \\
1,000 \\
\text { people }\end{array}$ \\
\hline 1900 & 0.11 & 1922 & 111.53 & 1944 & 220.23 & 1966 & 489.34 & 1988 & 772.92 \\
\hline 1901 & 0.19 & 1923 & 134.90 & 1945 & 221.80 & 1967 & 500.66 & 1989 & 776.99 \\
\hline 1902 & 0.29 & 1924 & 154.35 & 1946 & 243.11 & 1968 & 516.49 & 1990 & 773.40 \\
\hline 1903 & 0.41 & 1925 & 173.26 & 1947 & 262.56 & 1969 & 533.37 & 1991 & 760.19 \\
\hline 1904 & 0.67 & 1926 & 189.10 & 1948 & 280.20 & 1970 & 545.35 & 1992 & 757.96 \\
\hline 1905 & 0.94 & 1927 & 195.77 & 1949 & 299.56 & 1971 & 562.45 & 1993 & 761.94 \\
\hline 1906 & 1.27 & 1928 & 204.87 & 1950 & 323.71 & 1972 & 585.60 & 1994 & 766.94 \\
\hline 1907 & 1.65 & 1929 & 219.31 & 1951 & 337.14 & 1973 & 615.19 & 1995 & 770.99 \\
\hline 1908 & 2.24 & 1930 & 217.34 & 1952 & 340.57 & 1974 & 632.32 & 1996 & 781.16 \\
\hline 1909 & 3.45 & 1931 & 210.37 & 1953 & 353.67 & 1975 & 640.07 & 1997 & 776.02 \\
\hline 1910 & 5.07 & 1932 & 195.38 & 1954 & 361.40 & 1976 & 659.47 & 1998 & 781.20 \\
\hline 1911 & 6.81 & 1933 & 192.38 & 1955 & 379.77 & 1977 & 669.03 & 1999 & 790.07 \\
\hline 1912 & 9.90 & 1934 & 199.90 & 1956 & 387.58 & 1978 & 690.17 & 2000 & 800.30 \\
\hline 1913 & 12.94 & 1935 & 208.61 & 1957 & 392.11 & 1979 & 700.42 & 2001 & 825.49 \\
\hline 1914 & 17.79 & 1936 & 222.62 & 1958 & 392.17 & 1980 & 710.71 & 2002 & 815.22 \\
\hline 1915 & 24.77 & 1937 & 233.33 & 1959 & 402.83 & 1981 & 715.22 & 2003 & 815.50 \\
\hline 1916 & 35.48 & 1938 & 229.65 & 1960 & 410.37 & 1982 & 713.95 & 2004 & 829.26 \\
\hline 1917 & 49.57 & 1939 & 236.93 & 1961 & 415.11 & 1983 & 724.30 & 2005 & 836.58 \\
\hline 1918 & 59.69 & 1940 & 245.63 & 1962 & 426.06 & 1984 & 728.20 & 2006 & 840.09 \\
\hline 1919 & 72.50 & 1941 & 261.57 & 1963 & 438.75 & 1985 & 744.50 & 2007 & 843.57 \\
\hline 1920 & 86.78 & 1942 & 244.73 & 1964 & 451.57 & 1986 & 753.33 & 2008 & 840.80 \\
\hline 1921 & 96.68 & 1943 & 225.89 & 1965 & 466.90 & 1987 & 758.58 & 2009 & 828.04 \\
\hline
\end{tabular}




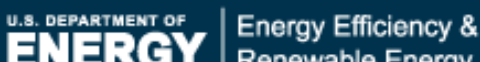 \\ Renewable Energy}

\section{Vehicle Technologies Program}

\section{Fact \#698: October 24, 2011 Changes in the Federal Highway Administration Vehicle Travel Data}

With the April release of Table VM-1 from Highway Statistics 2009 came several changes to the availability of data on vehicle miles of travel (VMT). From 1966 to 2008, the Federal Highway Administration (FHWA) published the VMT, fuel use, and average fuel economy of 2-axle, 4-tire trucks separately from cars on Table VM-1. The 2-axle, 4tire truck category included pickups, vans, sport utility vehicles, and other 4-tire trucks. For 2009 data and thereafter, car and 2-axle, 4-tire truck data are aggregated together and reported by the length of the vehicle's wheelbase (short and long). In addition, significant methodology changes were made to the single-unit truck and the combination truck data on VM-1, such that the data are not comparable with the previous years' data. Knowing that comparability would be an issue, the FHWA released revised versions of Table VM-1 going back to the year 2007. You can find the revised data in the Highway Statistics on the U.S. Department of Transportation Federal Highway Administration web site.

The 2008 VM-1 Original Table

\begin{tabular}{|c|c|c|c|c|c|c|c|c|c|c|}
\hline \multicolumn{11}{|c|}{$\begin{array}{c}\text { Annual Vehicle Distance Traveled in Miles and Related Data - } 2008 \text { 1/ } \\
\text { by Highway Category and Vehicle Type } \\
\text { December } 2009 \\
\text { Table VM-1 }\end{array}$} \\
\hline \multirow[b]{2}{*}{ YEAR } & \multirow[b]{2}{*}{ ITEM } & \multirow[b]{2}{*}{$\begin{array}{c}\text { PASSENGER } \\
\text { CARS }\end{array}$} & \multirow[b]{2}{*}{$\begin{array}{l}\text { MOTOR- } \\
\text { CYCLES }\end{array}$} & \multirow[b]{2}{*}{ BUSES } & \multirow[b]{2}{*}{$\begin{array}{l}\text { OTHER 2- } \\
\text { AXLE 4- } \\
\text { TIRE } \\
\text { VEHICLES } \\
3 l\end{array}$} & \multirow[b]{2}{*}{$\begin{array}{l}\text { SINGLE- } \\
\text { UNIT 2- } \\
\text { AXLE 6- } \\
\text { TIRE OR } \\
\text { MORE } \\
\text { TRUCKS 4I }\end{array}$} & \multirow[b]{2}{*}{$\begin{array}{l}\text { COMBINATION } \\
\text { TRUCKS }\end{array}$} & \multicolumn{2}{|c|}{ SUBTOTALS } & \multirow[b]{2}{*}{$\begin{array}{l}\text { ALL } \\
\text { MOTOR } \\
\text { VEHICLES } \\
2 l\end{array}$} \\
\hline & & & & & & & & $\begin{array}{l}\text { PASSENGER } \\
\text { CARS AND } \\
\text { OTHER 2- } \\
\text { AXLE 4-TIRE } \\
\text { VEHICLES }\end{array}$ & $\begin{array}{l}\text { SINGE-UNIT 2- } \\
\text { AXLE 6-TIRE OR } \\
\text { MORE AND } \\
\text { COMBINATION } \\
\text { TRUCKS }\end{array}$ & \\
\hline & \multicolumn{10}{|c|}{ Motor-Vehicle Travel: (millions of vehicle-miles) } \\
\hline 2008 & \multirow{2}{*}{$\begin{array}{l}\text { Interstate } \\
\text { Rural }\end{array}$} & 115,532 & 1,348 & 1,027 & 77,842 & 7,299 & 40,242 & 193,373 & 47,542 & 243,290 \\
\hline 2007 & & 122,183 & 1,420 & 986 & 82,030 & 7,188 & 42,632 & 204,212 & 49,819 & 256,438 \\
\hline 2008 & \multirow{2}{*}{$\begin{array}{l}\text { Other Arterial } \\
\text { Rural }\end{array}$} & 191,897 & 2,418 & 1,020 & 139,867 & 13,646 & 25,426 & 331,764 & 39,071 & 374,273 \\
\hline 2007 & & 204,123 & 2,305 & 1,015 & 145,985 & 13,877 & 26,160 & 350,108 & 40,037 & 393,465 \\
\hline 2008 & \multirow{2}{*}{ Other Rural } & 195,684 & 1,929 & 1,772 & 144,171 & 15,478 & 13,820 & 339,855 & 29,298 & 372,855 \\
\hline 2007 & & 203,485 & 1,820 & 1,722 & 148,612 & 15,659 & 14,101 & 352,097 & 29,760 & 385,400 \\
\hline 2008 & \multirow{2}{*}{ All Rural } & 503,112 & 5,695 & 3,819 & 361,880 & 36,423 & 79,488 & 864,993 & 115,911 & 990,418 \\
\hline 2007 & & 529,791 & 5,546 & 3,723 & 376,627 & 36,723 & 82,893 & 906,418 & 119,616 & $1,035,303$ \\
\hline 2008 & \multirow{2}{*}{$\begin{array}{l}\text { Interstate } \\
\text { Urban }\end{array}$} & 262,321 & 2,738 & 1,077 & 169,605 & 10,127 & 30,223 & 431,926 & 40,350 & 476,091 \\
\hline 2007 & & 267,559 & 2,631 & 1,052 & 170,669 & 10,143 & 31,262 & 438,228 & 41,405 & 483,315 \\
\hline 2008 & \multirow{2}{*}{ Other Urban } & 850,417 & 6,051 & 2,218 & 577,117 & 37,400 & 33,797 & $1,427,534$ & 71,197 & $1,507,000$ \\
\hline 2007 & & 875,118 & 5,444 & 2,205 & 564,975 & 35,147 & 30,892 & $1,440,093$ & 66,039 & $1,513,781$ \\
\hline
\end{tabular}




\begin{tabular}{|c|c|c|c|c|c|c|c|c|c|c|}
\hline \multicolumn{11}{|c|}{$\begin{array}{c}\text { Annual Vehicle Distance Traveled in Miles and Related Data - } 20081 \text { 1/ } \\
\text { by Highway Category and Vehicle Type } \\
\text { December } 2009 \\
\text { Table VM-1 }\end{array}$} \\
\hline \multirow[b]{2}{*}{ YEAR } & \multirow[b]{2}{*}{ ITEM } & \multirow[b]{2}{*}{$\begin{array}{l}\text { PASSENGER } \\
\text { CARS }\end{array}$} & \multirow[b]{2}{*}{$\begin{array}{l}\text { MOTOR- } \\
\text { CYCLES }\end{array}$} & \multirow[b]{2}{*}{ BUSES } & \multirow[b]{2}{*}{$\begin{array}{l}\text { OTHER 2- } \\
\text { AXLE 4- } \\
\text { TIRE } \\
\text { VEHICLES } \\
3 l\end{array}$} & \multirow[b]{2}{*}{$\begin{array}{l}\text { SINGLE- } \\
\text { UNIT 2- } \\
\text { AXLE 6- } \\
\text { TIRE OR } \\
\text { MORE } \\
\text { TRUCKS 4I }\end{array}$} & \multirow[b]{2}{*}{$\begin{array}{l}\text { COMBINATION } \\
\text { TRUCKS }\end{array}$} & \multicolumn{2}{|c|}{ SUBTOTALS } & \multirow[b]{2}{*}{$\begin{array}{l}\text { ALL } \\
\text { MOTOR } \\
\text { VEHICLES } \\
2 l\end{array}$} \\
\hline & & & & & & & & $\begin{array}{l}\text { PASSENGER } \\
\text { CARS AND } \\
\text { OTHER 2- } \\
\text { AXLE 4-TIRE } \\
\text { VEHICLES }\end{array}$ & $\begin{array}{l}\text { SINGE-UNIT 2- } \\
\text { AXLE 6-TIRE OR } \\
\text { MORE AND } \\
\text { COMBINATION } \\
\text { TRUCKS }\end{array}$ & \\
\hline & \multicolumn{10}{|c|}{ Motor-Vehicle Travel: (millions of vehicle-miles) } \\
\hline 2008 & \multirow{2}{*}{ All Urban } & $1,112,738$ & 8,789 & 3,295 & 746,722 & 47,527 & 64,019 & $1,859,460$ & 111,547 & $1,983,091$ \\
\hline 2007 & & $1,142,677$ & 8,075 & 3,257 & 735,644 & 45,290 & 62,153 & $1,878,320$ & 107,444 & $1,997,096$ \\
\hline 2008 & \multirow{2}{*}{$\begin{array}{l}\text { Total Rural } \\
\text { and Urban }\end{array}$} & $1,615,850$ & 14,484 & 7,114 & $1,108,603$ & 83,951 & 143,507 & $2,724,453$ & 227,458 & $2,973,509$ \\
\hline 2007 & & $1,672,467$ & 13,621 & 6,980 & $1,112,271$ & 82,014 & 145,046 & $2,784,738$ & 227,060 & $3,032,399$ \\
\hline 2008 & \multirow{2}{*}{$\begin{array}{l}\text { Number of } \\
\text { motor } \\
\text { vehicles } \\
\text { registered } 5 /\end{array}$} & $137,079,843$ & $7,752,926$ & 843,308 & $101,234,849$ & $6,790,882$ & $2,215,856$ & $238,314,692$ & $9,006,738$ & $255,917,664$ \\
\hline 2007 & & $135,932,930$ & $7,138,476$ & 834,436 & $101,469,615$ & $6,806,630$ & $2,220,995$ & $237,402,545$ & $9,027,624$ & $254,403,081$ \\
\hline 2008 & \multirow{2}{*}{$\begin{array}{l}\text { Average } \\
\text { miles traveled } \\
\text { per vehicle }\end{array}$} & 11,788 & 1,868 & 8,436 & 10,951 & 12,362 & 64,764 & 11,432 & 25,254 & 11,619 \\
\hline 2007 & & 12,304 & 1,908 & 8,365 & 10,962 & 12,049 & 65,307 & 11,730 & 25,152 & 11,920 \\
\hline 2008 & \multirow{2}{*}{$\begin{array}{l}\text { Person-miles } \\
\text { of travel } 6 / \\
\text { (millions) }\end{array}$} & $2,553,043$ & 18,395 & 150,827 & $1,921,960$ & 83,951 & 143,507 & $4,475,004$ & 227,458 & $4,871,683$ \\
\hline 2007 & & $2,642,498$ & 17,298 & 147,985 & $1,928,319$ & 82,014 & 145,046 & $4,570,818$ & 227,060 & $4,963,161$ \\
\hline 2008 & \multirow{2}{*}{$\begin{array}{l}\text { Fuel } \\
\text { consumed } 7 / \\
\text { (thousand } \\
\text { gallons) }\end{array}$} & $71,497,204$ & 256,358 & $1,109,636$ & $61,198,934$ & $9,888,729$ & $26,814,441$ & $132,696,139$ & $36,703,170$ & $170,765,303$ \\
\hline 2007 & & $74,377,197$ & 242,241 & $1,144,861$ & $61,836,216$ & $10,043,778$ & $28,545,442$ & $136,213,413$ & $38,589,220$ & $176,189,735$ \\
\hline 2008 & \multirow{2}{*}{$\begin{array}{l}\text { Average fuel } \\
\text { consumption } \\
\text { per vehicle } \\
\text { (gallons) } 7 /\end{array}$} & 522 & 33 & 1,316 & 605 & 1,456 & 12,101 & 557 & 4,075 & 667 \\
\hline 2007 & & 547 & 34 & 1,372 & 609 & 1,476 & 12,853 & 574 & 4,275 & 693 \\
\hline 2008 & \multirow{2}{*}{$\begin{array}{l}\text { Average } \\
\text { miles traveled } \\
\text { per gallon of } \\
\text { fuel } \\
\text { consumed } 7 /\end{array}$} & 22.6 & 56.5 & 6.4 & 18.1 & 8.5 & 5.4 & 20.5 & 6.2 & 17.4 \\
\hline 2007 & & 22.5 & 56.2 & 6.1 & 18.0 & 8.2 & 5.1 & 20.4 & 5.9 & 17.2 \\
\hline \multicolumn{11}{|c|}{$\begin{array}{l}\text { 1/ The } 50 \text { states and the District of Columbia report travel by highway category, number of motor vehicles registered, and total fuel consumed. The travel and fuel data by } \\
\text { vehicle type and stratification of trucks are estimated by the Federal Highway Administration (FHWA). Estimation procedures include use of State supplied data, the } 2002 \\
\text { Census of Transportation Vehicle Inventory and Use Survey (VIUS), and other sources. } \\
\text { 2/ Totals by highway category are from table VM-2. Some changes between rural and urban roadways can be attributed to } 2002 \text { census boundary changes. } \\
\text { 3/ Other 2-Axle 4-Tire Vehicles which are not passenger cars. These include vans, pickup trucks, and sport/utility vehicles. } \\
\text { 4/ Single-Unit 2-Axle 6-Tire or More Trucks on a single frame with at least two axles and six tires. } \\
5 \text { / Truck registration figures are from tables MV-1 and MV-9 with truck distribution estimated by the FHWA. } \\
\text { 6/ Vehicle occupancy is estimated by the FHWA from the } 2001 \text { National Household Travel Survey (NHTS); For heavy trucks, } 1 \text { motor vehicle miles traveled = } 1 \text { person-miles } \\
\text { traveled. } \\
\text { 7/ Total fuel consumption figures are from tables MF-21 and MF-27. Distribution by vehicle type is estimated by the FHWA based on miles per gallon for both diesel and } \\
\text { gasoline powered vehicles using State-supplied data, the } 2002 \text { VIUS, and other sources with nominal inputs for motorcycles and buses. }\end{array}$} \\
\hline
\end{tabular}


The 2009 VM-1 Revised Methodology Table

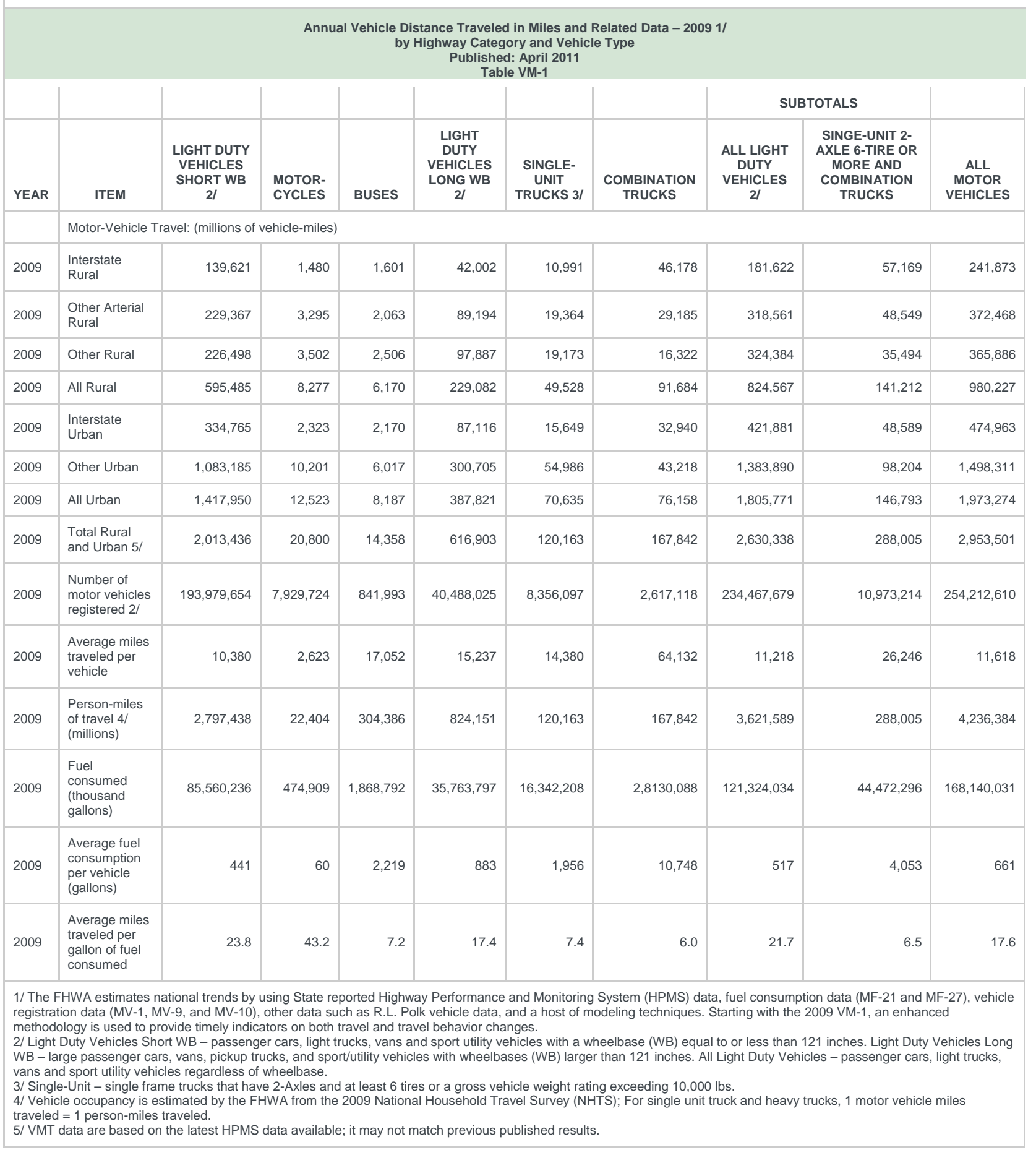

\section{Supporting Information}

U.S. Department of Transportation, Federal Highway Administration, Highway Statistics 2009. 


\section{u.s. oppaprusur of | Energy Efficiency \& \\ Renewable Energy}

\section{Vehicle Technologies Program}

Fact \#699: October 31, 2011

Transportation Energy Use by Mode and Fuel Type, 2009

Highway vehicles are responsible for most of the energy consumed by the transportation sector. Most of the fuel used in light vehicles is gasoline, while most of the fuel used in med/heavy trucks and buses is diesel.

Transportation Energy Use by Mode and Fuel Type, 2009

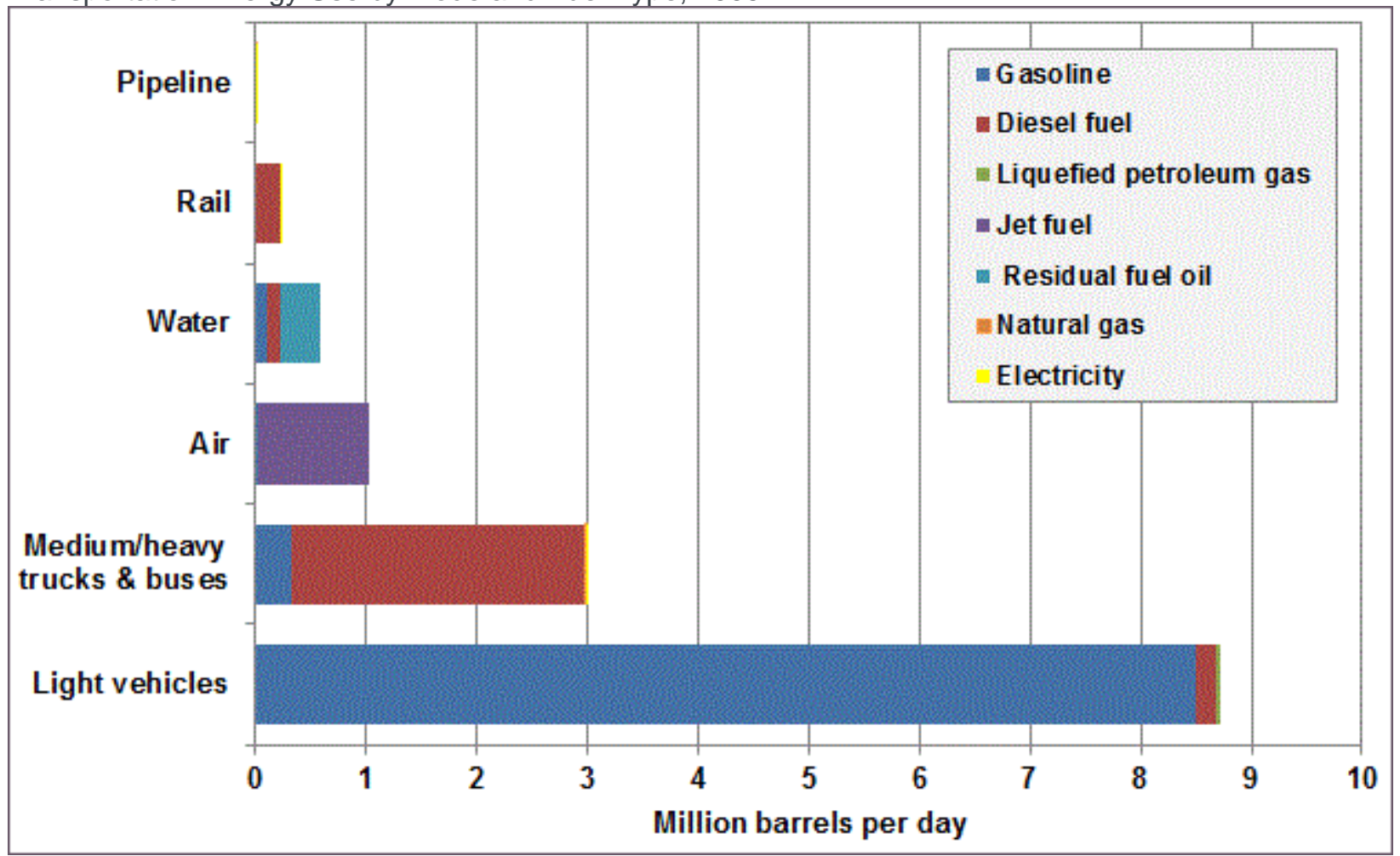




\section{Supporting Information}

\begin{tabular}{|c|c|c|c|c|c|c|c|c|}
\hline \multicolumn{9}{|c|}{ Transportation Energy Use by Mode and Fuel Type, 2009} \\
\hline & Gasoline & $\begin{array}{c}\text { Diesel } \\
\text { fuel }\end{array}$ & $\begin{array}{l}\text { Liquefied } \\
\text { petroleum } \\
\text { gas }\end{array}$ & $\begin{array}{l}\text { Jet } \\
\text { fuel }\end{array}$ & $\begin{array}{c}\text { Residual } \\
\text { fuel oil }\end{array}$ & $\begin{array}{c}\text { Natural } \\
\text { gas }\end{array}$ & Electricity & Total \\
\hline Light vehicles & 8.5 & 0.2 & 0.0 & 0.0 & 0.0 & 0.0 & 0.0 & 8.7 \\
\hline $\begin{array}{l}\text { Medium/heavy } \\
\text { trucks \& } \\
\text { buses }\end{array}$ & 0.3 & 2.6 & 0.0 & 0.0 & 0.0 & 0.0 & 0.0 & 3.0 \\
\hline Air & 0.0 & 0.0 & 0.0 & 1.0 & 0.0 & 0.0 & 0.0 & 1.0 \\
\hline Water & 0.1 & 0.1 & 0.4 & 0.0 & 0.0 & 0.0 & 0.0 & 0.6 \\
\hline Rail & 0.0 & 0.2 & 0.0 & 0.0 & 0.0 & 0.0 & 0.0 & 0.2 \\
\hline Pipelines & 0.0 & 0.0 & 0.0 & 0.0 & 0.0 & 0.007 & 0.003 & 0.0 \\
\hline $\begin{array}{l}\text { TOTAL } \\
\text { Highway \& } \\
\text { Nonhighway }\end{array}$ & 8.9 & 3.2 & 0.4 & 1.0 & 0.0 & 0.0 & 0.0 & 13.6 \\
\hline $\begin{array}{l}\text { Notes: Civilian c } \\
\text { not include all sol } \\
\text { Source: Oak Ric } \\
\text { June } 2011 .\end{array}$ & $\begin{array}{l}\text { onsumption } \\
\text { urces of trans } \\
\text { dge National }\end{array}$ & $\begin{array}{l}\text { only. Air } \\
\text { sportatior } \\
\text { Laborato }\end{array}$ & $\begin{array}{l}\text { includes fuel u } \\
\text { ר, e.g. snowme } \\
\text { ry, Transporta }\end{array}$ & $\begin{array}{l}\text { use for } \\
\text { obiles. }\end{array}$ & $\begin{array}{l}\text { half of all int } \\
\text { nergy Data B }\end{array}$ & ernational & $\begin{array}{l}\text { flights. Total } r \\
\text { on } 30 \text {, ORNL- }\end{array}$ & $\begin{array}{l}\text { nay } \\
6986,\end{array}$ \\
\hline
\end{tabular}




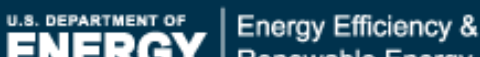 \\ Renewable Energy}

\section{Vehicle Technologies Program}

\section{Fact \#700: November 7, 2011}

\section{Biodiesel Consumption is on the Rise for 2011}

The U.S. Energy Information Administration began tracking biodiesel consumption in 2001. For the first few years biodiesel consumption remained relatively low - well under one thousand barrels per year. Beginning with 2005 the consumption of biodiesel began to increase dramatically and peaked at a high of 8.5 million barrels per year in 2007 . The consumption of biodiesel slowed in 2010 due to U.S. economic conditions and the discontinuation of a Federal biodiesel producer tax credit. The credit, which expired in December 2009, was reinstated in December 2010. With the recovering economy and the tax credit returned, biodiesel consumption has rallied, exceeding 2010 levels in just the first six months of 2011.

Biodiesel Consumption, 2001-2011*

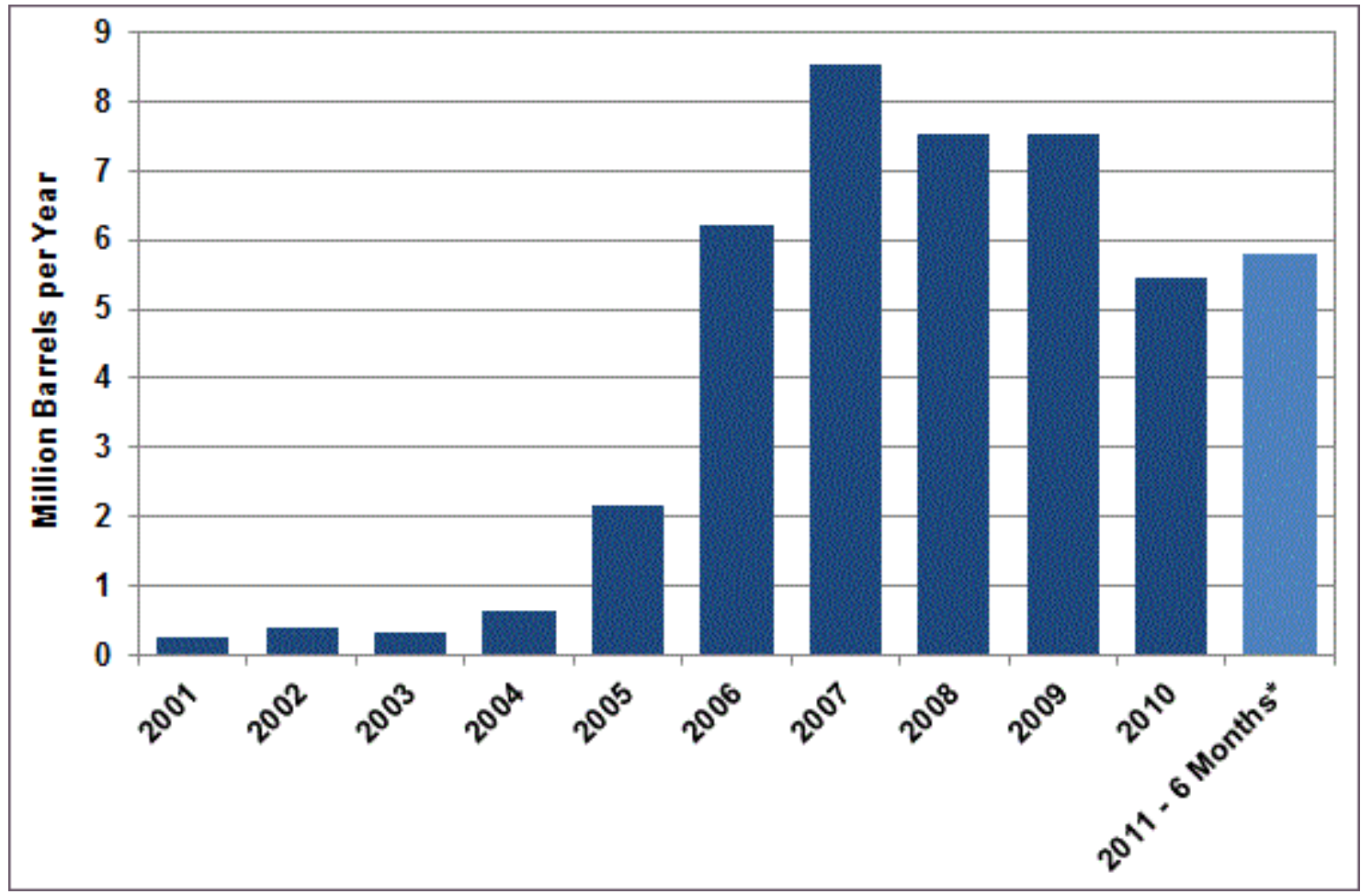

* 2011 figure is for the first 6 months. 


\section{Supporting Information}

\begin{tabular}{|c|c|}
\hline \multicolumn{2}{|c|}{ Biodiesel Consumption 2001-2011* } \\
\hline Year & $\begin{array}{l}\text { Biodiesel Consumption } \\
\text { (Million Barrels) }\end{array}$ \\
\hline 2001 & 0.243 \\
\hline 2002 & 0.385 \\
\hline 2003 & 0.322 \\
\hline 2004 & 0.640 \\
\hline 2005 & 2.163 \\
\hline 2006 & 6.204 \\
\hline 2007 & 8.528 \\
\hline 2008 & 7.519 \\
\hline 2009 & 7.537 \\
\hline 2010 & 5.447 \\
\hline 20116 Months* & 5.807 \\
\hline \multicolumn{2}{|c|}{ * 2011 figure is for the first 6 months. } \\
\hline \multicolumn{2}{|c|}{$\begin{array}{l}\text { Source: U.S. Energy Information Administration, } \\
\text { Monthly Energy Review, September 2011, Table } \\
\text { 10.4. } \\
\text { Additional resources: National Biodiesel Board, } \\
\text { Letter at Biodiesel Conference website and The }\end{array}$} \\
\hline
\end{tabular}




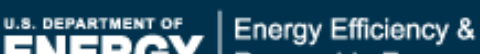 \\ Renewable Energy}

\section{Vehicle Technologies Program}

\section{Fact \#701: November 14, 2011 How Much More Would You Pay for an Electric Vehicle?}

A survey conducted worldwide between November 2010 and May 2011 asked respondents from 17 different countries questions about their willingness to purchase electric vehicles. More than half of the respondents in 14 of those countries said they would buy an electric vehicle only if the vehicle price were the same or less than a similar gasoline vehicle. In the US, only $28 \%$ of respondents were willing to pay an extra amount for an electric vehicle; $65 \%$ indicated they would only buy an EV if it were the same price or less (another 7\% responded "Don't know"). India, Korea and China had the highest shares of respondents willing to pay extra.

Question: How much more would you be willing to pay for an electric vehicle compared to a similar vehicle with a gasoline engine?

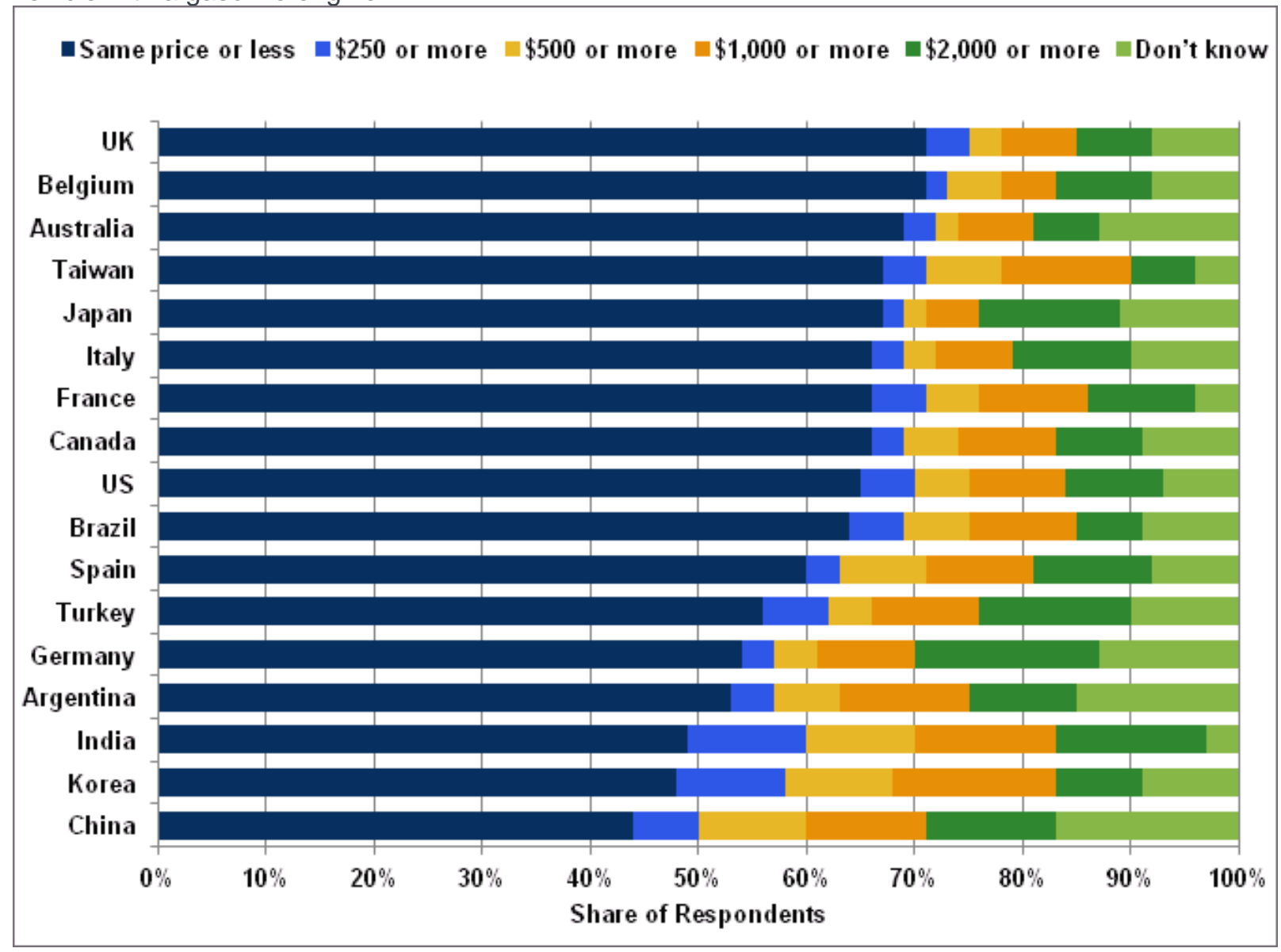

Note: All currency amounts are in U.S. dollars. 


\section{Supporting Information}

Question: How much more would you be willing to pay for an electric vehicle compared to a similar vehicle with a gasoline engine?

(Share of Respondents)

\begin{tabular}{|c|c|c|c|c|c|c|c|}
\hline Country & $\begin{array}{c}\text { Same } \\
\text { price } \\
\text { or } \\
\text { less }\end{array}$ & $\begin{array}{c}\$ 250 \\
\text { or } \\
\text { more }\end{array}$ & $\begin{array}{c}\$ 500 \\
\text { or } \\
\text { more }\end{array}$ & $\begin{array}{c}\$ 1,000 \\
\text { or } \\
\text { more }\end{array}$ & $\begin{array}{c}\$ 2,000 \\
\text { or } \\
\text { more }\end{array}$ & $\begin{array}{l}\text { Don't } \\
\text { know }\end{array}$ & Total \\
\hline UK & $71 \%$ & $4 \%$ & $3 \%$ & $7 \%$ & $7 \%$ & $8 \%$ & $100 \%$ \\
\hline Belgium & $71 \%$ & $2 \%$ & $5 \%$ & $5 \%$ & $9 \%$ & $8 \%$ & $100 \%$ \\
\hline Australia & $69 \%$ & $3 \%$ & $2 \%$ & $7 \%$ & $6 \%$ & $13 \%$ & $100 \%$ \\
\hline Taiwan & $67 \%$ & $4 \%$ & $7 \%$ & $12 \%$ & $6 \%$ & $4 \%$ & $100 \%$ \\
\hline Japan & $67 \%$ & $2 \%$ & $2 \%$ & $5 \%$ & $13 \%$ & $11 \%$ & $100 \%$ \\
\hline Canada & $66 \%$ & $3 \%$ & $5 \%$ & $9 \%$ & $8 \%$ & $9 \%$ & $100 \%$ \\
\hline France & $66 \%$ & $5 \%$ & $5 \%$ & $10 \%$ & $10 \%$ & $4 \%$ & $100 \%$ \\
\hline Italy & $66 \%$ & $3 \%$ & $3 \%$ & $7 \%$ & $11 \%$ & $10 \%$ & $100 \%$ \\
\hline US & $65 \%$ & $5 \%$ & $5 \%$ & $9 \%$ & $9 \%$ & $7 \%$ & $100 \%$ \\
\hline Brazil & $64 \%$ & $5 \%$ & $6 \%$ & $10 \%$ & $6 \%$ & $9 \%$ & $100 \%$ \\
\hline Spain & $60 \%$ & $3 \%$ & $8 \%$ & $10 \%$ & $11 \%$ & $8 \%$ & $100 \%$ \\
\hline Turkey & $56 \%$ & $6 \%$ & $4 \%$ & $10 \%$ & $14 \%$ & $10 \%$ & $100 \%$ \\
\hline Germany & $54 \%$ & $3 \%$ & $4 \%$ & $9 \%$ & $17 \%$ & $13 \%$ & $100 \%$ \\
\hline Argentina & $53 \%$ & $4 \%$ & $6 \%$ & $12 \%$ & $10 \%$ & $15 \%$ & $100 \%$ \\
\hline India & $49 \%$ & $11 \%$ & $10 \%$ & $13 \%$ & $14 \%$ & $3 \%$ & $100 \%$ \\
\hline Korea & $48 \%$ & $10 \%$ & $10 \%$ & $15 \%$ & $8 \%$ & $9 \%$ & $100 \%$ \\
\hline China & $44 \%$ & $6 \%$ & $10 \%$ & $11 \%$ & $12 \%$ & $17 \%$ & $100 \%$ \\
\hline
\end{tabular}

Note: All currency amounts are in U.S. dollars.

Source: Deloitte World EV Survey, N=over 13,000 respondents in 17 countries, conducted November 2010 - May 2011. 


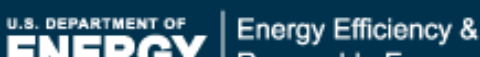 \\ Renewable Energy}

\section{Vehicle Technologies Program}

\section{Fact \#702: November 21, 2011 Consumer Preferences on Electric Vehicle Charging}

Data from a survey conducted between November 2010 and May 2011 show consumer preferences on electric vehicle (EV) charging times. Respondents from 17 different countries were asked for their longest acceptable charge time for an EV. In Taiwan, the country with the greatest number of respondents accepting longer charge times, nearly a third of the respondents said an 8-hour charge time was acceptable, while in Japan, the country with the least number of respondents accepting longer charge times, a third of the respondents answered 30 minutes or less. Currently, most EV batteries can charge within three to eight hours. Rapid charge systems can charge an EV within 30 minutes, but these systems are expensive, shorten the battery life, and put additional stress on the electricity grid.

Question: Considering your expected vehicle use, what is the longest time to fully recharge the battery that you would consider acceptable when buying or leasing an electric vehicle?

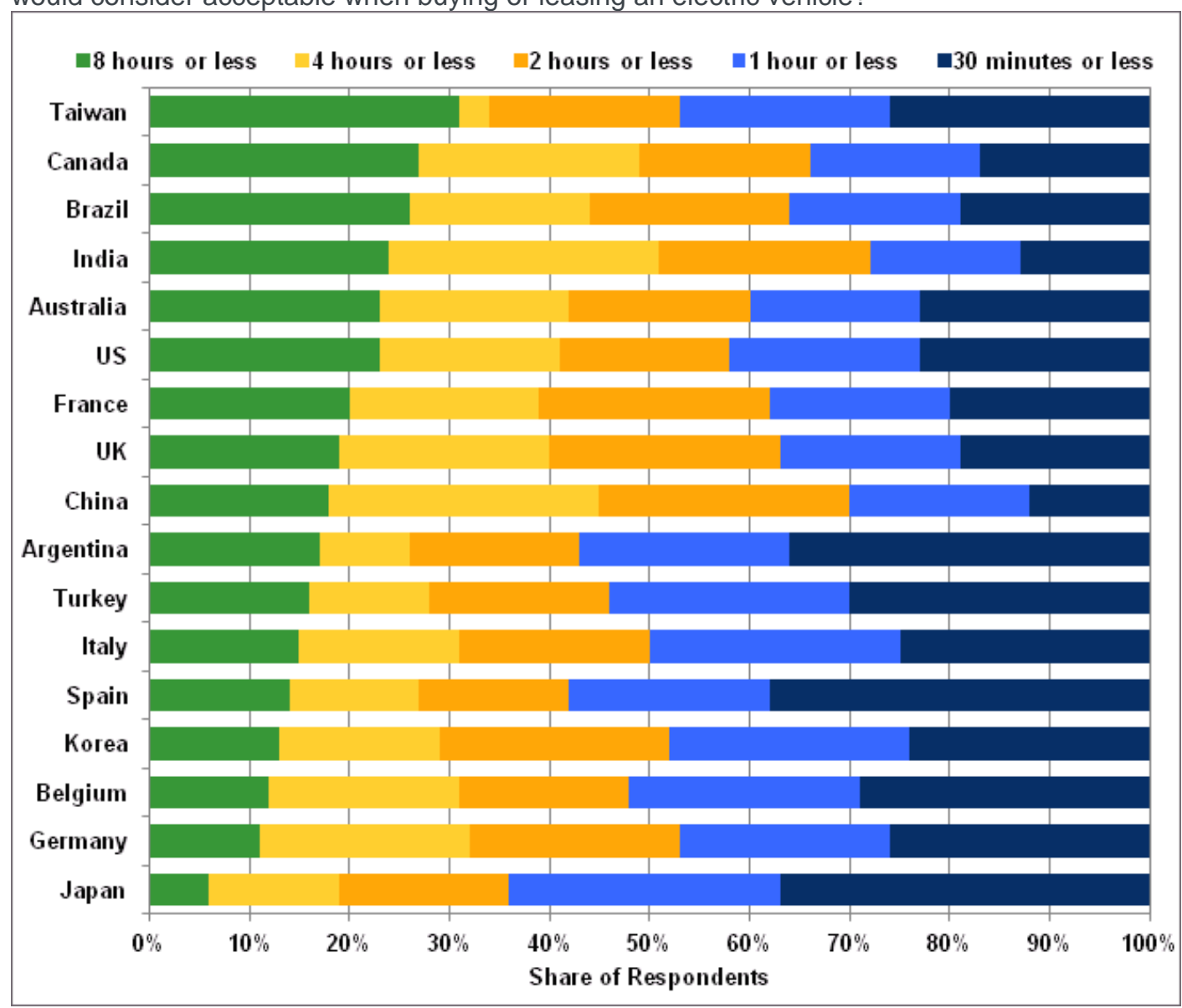




\section{Supporting Information}

\begin{tabular}{|c|c|c|c|c|c|c|}
\hline \multicolumn{7}{|c|}{$\begin{array}{l}\text { Question: Considering your expected vehicle use, } \\
\text { what is the longest time to fully recharge the battery } \\
\text { that you would consider acceptable when buying or } \\
\text { leasing an electric vehicle? }\end{array}$} \\
\hline Country & $\begin{array}{l}8 \\
\text { hours } \\
\text { or } \\
\text { less }\end{array}$ & $\begin{array}{l}4 \\
\text { hours } \\
\text { or } \\
\text { less }\end{array}$ & $\begin{array}{l}2 \\
\text { hours } \\
\text { or } \\
\text { less }\end{array}$ & $\begin{array}{l}1 \\
\text { hour } \\
\text { or } \\
\text { less }\end{array}$ & $\begin{array}{l}30 \\
\text { minutes } \\
\text { or } \\
\text { less }\end{array}$ & Total \\
\hline Tail & $\%$ & $3 \%$ & & 21 & $\%$ & $100 \%$ \\
\hline Can & 0 & $22 \%$ & $17 \%$ & $17 \%$ & $\%$ & $100 \%$ \\
\hline Brazil & $26 \%$ & $18 \%$ & $20 \%$ & $17 \%$ & $19 \%$ & $100 \%$ \\
\hline India & $24 \%$ & $27 \%$ & $21 \%$ & $15 \%$ & $13 \%$ & $100 \%$ \\
\hline US & $23 \%$ & $18 \%$ & $17 \%$ & $19 \%$ & $23 \%$ & $100 \%$ \\
\hline Australia & $23 \%$ & $19 \%$ & $18 \%$ & $17 \%$ & $23 \%$ & $100 \%$ \\
\hline France & $20 \%$ & $19 \%$ & $23 \%$ & $18 \%$ & $20 \%$ & $100 \%$ \\
\hline UK & $19 \%$ & $21 \%$ & 0 & $18 \%$ & $19 \%$ & $100 \%$ \\
\hline China & $18 \%$ & $27 \%$ & $25 \%$ & $18 \%$ & $12 \%$ & $100 \%$ \\
\hline Argentina & $17 \%$ & $9 \%$ & $17 \%$ & $21 \%$ & $36 \%$ & $100 \%$ \\
\hline Turkey & $16 \%$ & $12 \%$ & $18 \%$ & $24 \%$ & $30 \%$ & $100 \%$ \\
\hline Italy & $15 \%$ & $16 \%$ & $19 \%$ & $25 \%$ & $25 \%$ & $100 \%$ \\
\hline Spain & $14 \%$ & $13 \%$ & $15 \%$ & $20 \%$ & $38 \%$ & $100 \%$ \\
\hline Korea & $13 \%$ & $16 \%$ & $23 \%$ & $24 \%$ & $24 \%$ & $100 \%$ \\
\hline Belgium & $12 \%$ & $19 \%$ & $17 \%$ & $23 \%$ & $29 \%$ & $100 \%$ \\
\hline Germany & $11 \%$ & $21 \%$ & $21 \%$ & $21 \%$ & $26 \%$ & $100 \%$ \\
\hline Japan & $6 \%$ & $13 \%$ & $17 \%$ & $27 \%$ & $37 \%$ & $100 \%$ \\
\hline
\end{tabular}

Source: Deloitte, Unplugged: Electric vehicle realities versus consumer expectations se, 2011. 


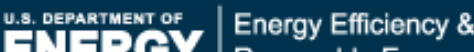 \\ Renewable Energy}

\section{Vehicle Technologies Program}

Fact \#703: November 28, 2011

Hybrid Vehicles Lose Market Share in 2010

For the first time since hybrid vehicles entered the market, the share of hybrid registrations declined in 2010 - from $2.9 \%$ in 2009 to $2.6 \%$ in 2010. Reasons for this include the relatively lower price of gasoline and the availability of several non-hybrid vehicles that get 40 miles per gallon or more.

Share of Hybrid Registrations, 2005-2010

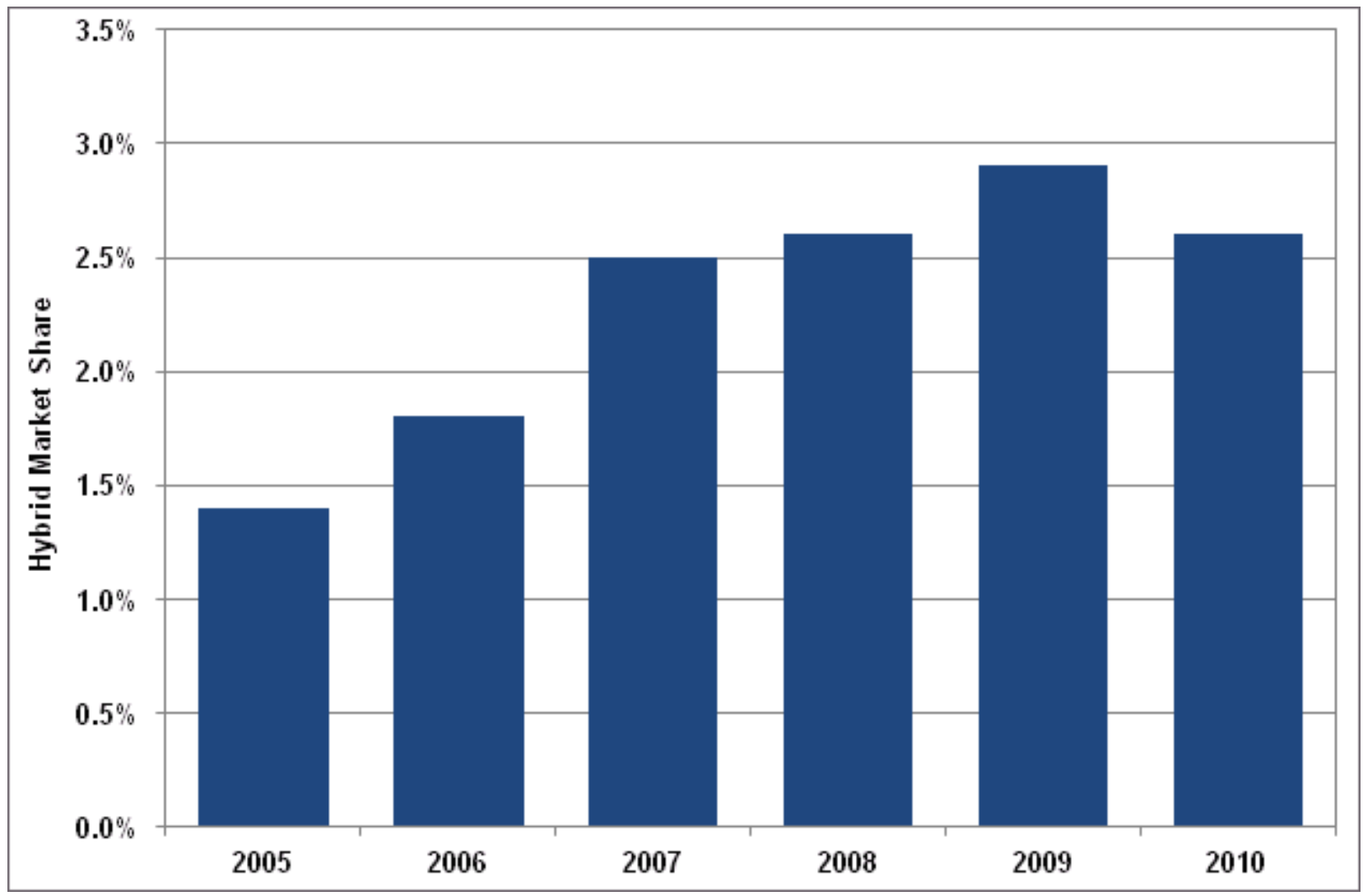




\section{Supporting Information}

Share of Hybrid Registrations, 2005-2010

\begin{tabular}{|c|c|}
\hline Calendar Year & Market Share \\
\hline 2005 & $1.4 \%$ \\
\hline 2006 & $1.8 \%$ \\
\hline 2007 & $2.5 \%$ \\
\hline 2008 & $2.6 \%$ \\
\hline 2009 & $2.9 \%$ \\
\hline 2010 & $2.6 \%$ \\
\hline
\end{tabular}

Source: R. L. Polk and Company, "U.S. Hybrid Market Share Suffers, Expected to Rebound," April 2011. 


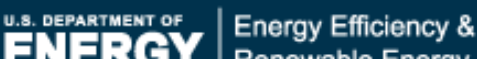 \\ Renewable Energy}

\section{Vehicle Technologies Program}

\section{Fact \#704: December 5, 2011}

\section{Fuel Consumption Standards for New Heavy Pickups and Vans}

In September 2011 the National Highway Traffic Safety Administration issued the final rule to set standards regulating the fuel use of new vehicles heavier than $8,500 \mathrm{lbs}$. gross vehicle weight. Included in the new standards are pickup trucks over 8,500 lbs., cargo trucks over 8,500 lbs., and passenger vans over 10,000 lbs. Standards were set separately for gasoline and diesel vehicles, on a scale that depends on a "work factor." The work factor, which is expressed in pounds, takes into account the vehicle's payload capacity, towing capacity, and whether or not the vehicle is four-wheel drive (see note below for work factor details). Standards for the years 2014 and 2015 are voluntary, but standards are mandatory thereafter.

Fuel Consumption Target Standards for Gasoline Heavy Pickups and Vans, 2014-2018

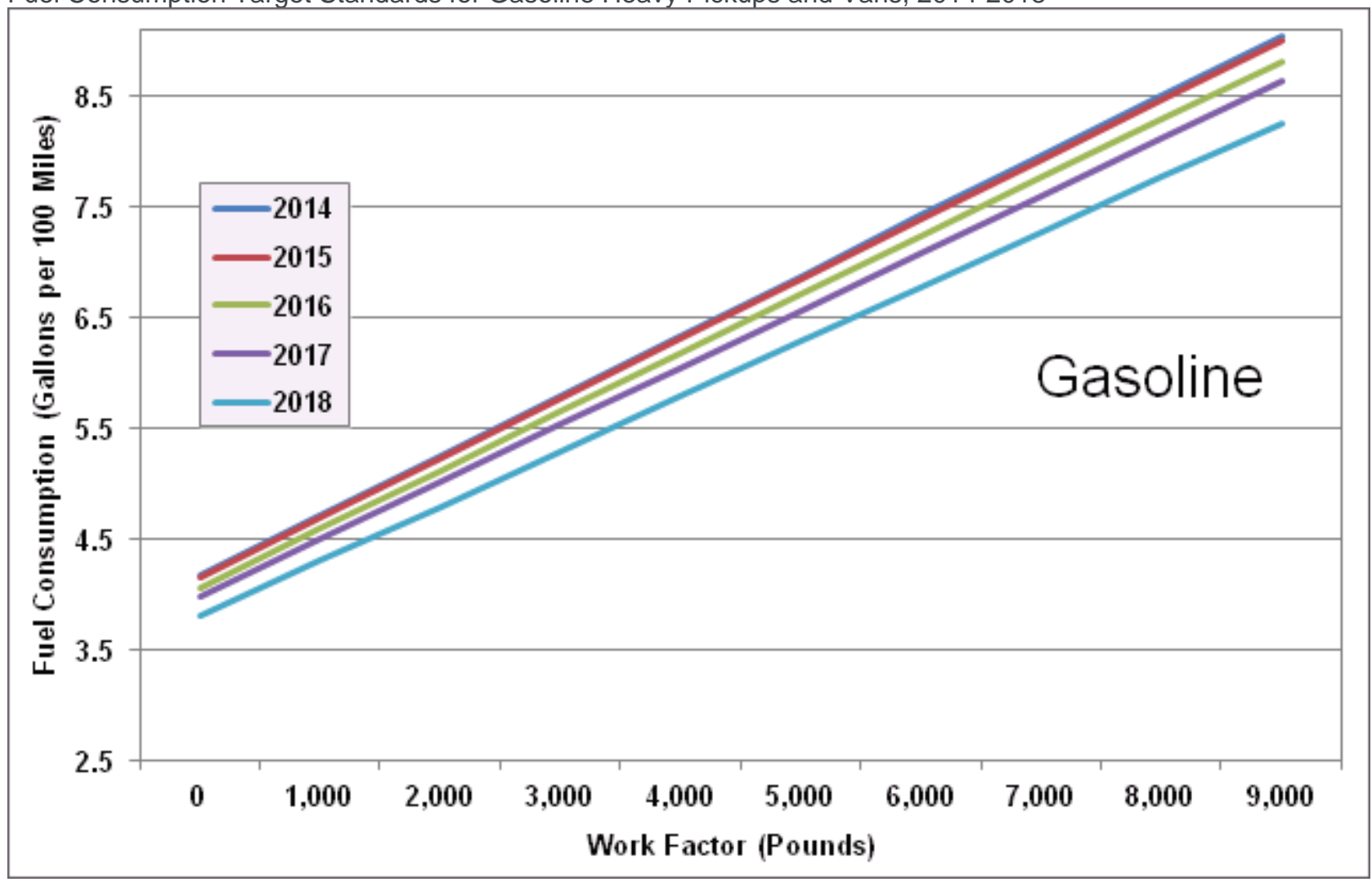


Fuel Consumption Target Standards for Diesel Heavy Pickups and Vans, 2014-2018

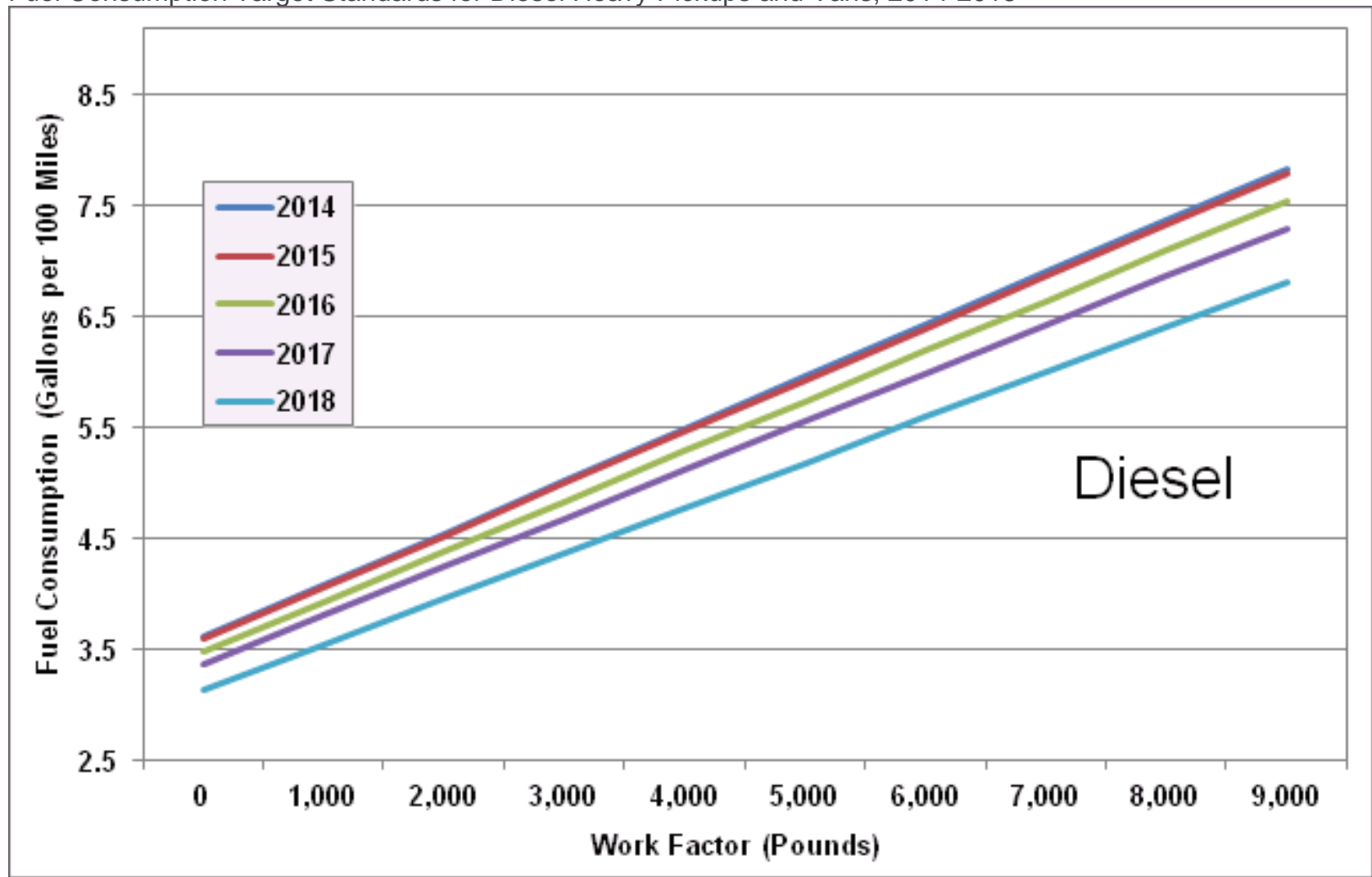

Note: Work factor is a weighted average of $25 \%$ towing capacity and $75 \%$ payload capacity. An additional $500 \mathrm{lbs}$. is added to payload capacity when the vehicle is four-wheel drive. 


\section{Supporting Information}

\begin{tabular}{|c|c|c|c|c|c|}
\hline $\begin{array}{l}\text { Work Factor } \\
\text { (pounds) }\end{array}$ & 2014 & 2015 & 2016 & 2017 & 2018 \\
\hline \multicolumn{6}{|c|}{ Gasoline Vehicles } \\
\hline 0 & 4.17 & 4.15 & 4.07 & 3.98 & 3.81 \\
\hline 1,000 & 4.71 & 4.69 & 4.60 & 4.50 & 4.31 \\
\hline 2,000 & 5.25 & 5.23 & 5.13 & 5.02 & 4.80 \\
\hline 3,000 & 5.80 & 5.77 & 5.65 & 5.53 & 5.30 \\
\hline 4,000 & 6.34 & 6.31 & 6.18 & 6.05 & 5.79 \\
\hline 5,000 & 6.88 & 6.85 & 6.71 & 6.57 & 6.29 \\
\hline 6,000 & 7.42 & 7.38 & 7.24 & 7.09 & 6.78 \\
\hline 7,000 & 7.96 & 7.92 & 7.77 & 7.61 & 7.28 \\
\hline 8,000 & 8.51 & 8.46 & 8.29 & 8.12 & 7.77 \\
\hline 9,000 & 9.05 & 9.00 & 8.82 & 8.64 & 8.27 \\
\hline \multicolumn{6}{|c|}{ Diesel Vehicles } \\
\hline 0 & 3.61 & 3.60 & 3.48 & 3.37 & 3.14 \\
\hline 1,000 & 4.08 & 4.07 & 3.93 & 3.81 & 3.55 \\
\hline 2,000 & 4.55 & 4.53 & 4.38 & 4.24 & 3.96 \\
\hline 3,000 & 5.02 & 5.00 & 4.84 & 4.68 & 4.37 \\
\hline 4,000 & 5.49 & 5.46 & 5.29 & 5.12 & 4.78 \\
\hline 5,000 & 5.96 & 5.93 & 5.74 & 5.56 & 5.19 \\
\hline 6,000 & 6.43 & 6.40 & 6.19 & 5.99 & 5.59 \\
\hline 7,000 & 6.9 & 6.86 & 6.64 & 6.43 & 6.00 \\
\hline 8,000 & 7.37 & 7.33 & 7.10 & 6.87 & 6.41 \\
\hline 9,000 & 7.84 & 7.79 & 7.55 & 7.30 & 6.82 \\
\hline
\end{tabular}

Note: This table shows targets by 1,000 -lb work factor increments. Actual standards move on a sliding scale as shown in the graph.

Source: Federal Register, Vol. 76, No. 179, September 15, 2011, pp. 25324 - 25728. 


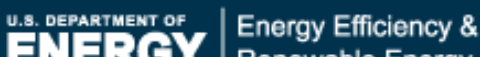 \\ Renewable Energy}

\section{Vehicle Technologies Program}

\section{Fact \#705: December 12, 2011}

\section{Fuel Consumption Standards for Combination Tractors}

The National Highway Traffic Safety Administration published a final rule setting fuel consumption standards for heavy trucks in September 2011. For tractor-trailers, the standards focus on the gallons of fuel per thousand ton-miles. Ton-miles are equal to the weight of a shipment transported multiplied by the distance hauled. Because differences in the tractors create differences in the fuel used, standards were set for varying roof height (low, mid, and high), gross vehicle weight rating (class 7 and 8), and types of tractor (day cab, sleeper cab).

Combination Tractor Fuel Consumption Standards, Model Years (MY) 2014-2017

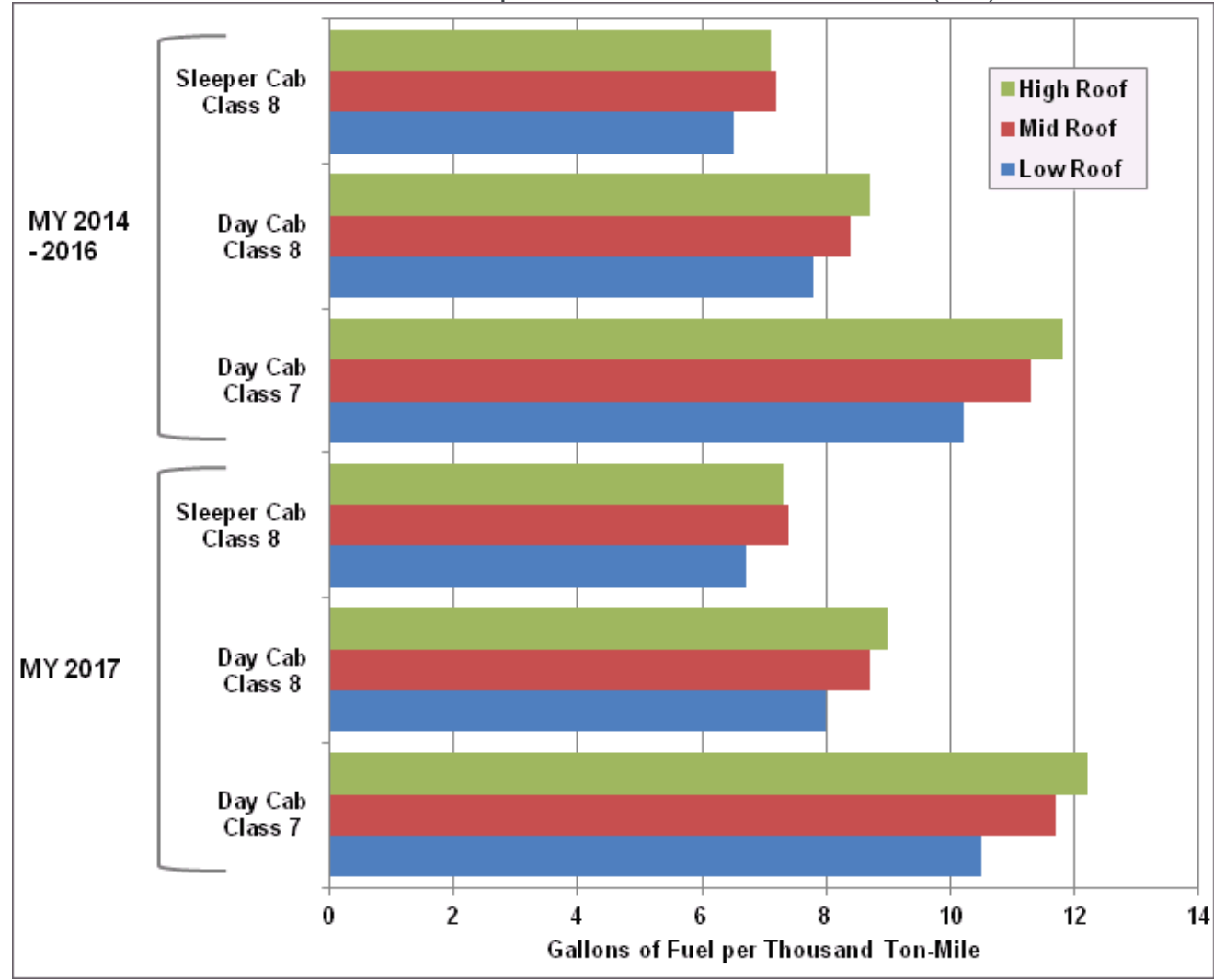

Note: The standards for 2014 and 2015 are voluntary.

Class 7 trucks have a gross vehicle weight rating between 26,000 and 33,000 lbs.

Class 8 trucks have a gross vehicle weight rating over 33,000 lbs. 


\section{Supporting Information}

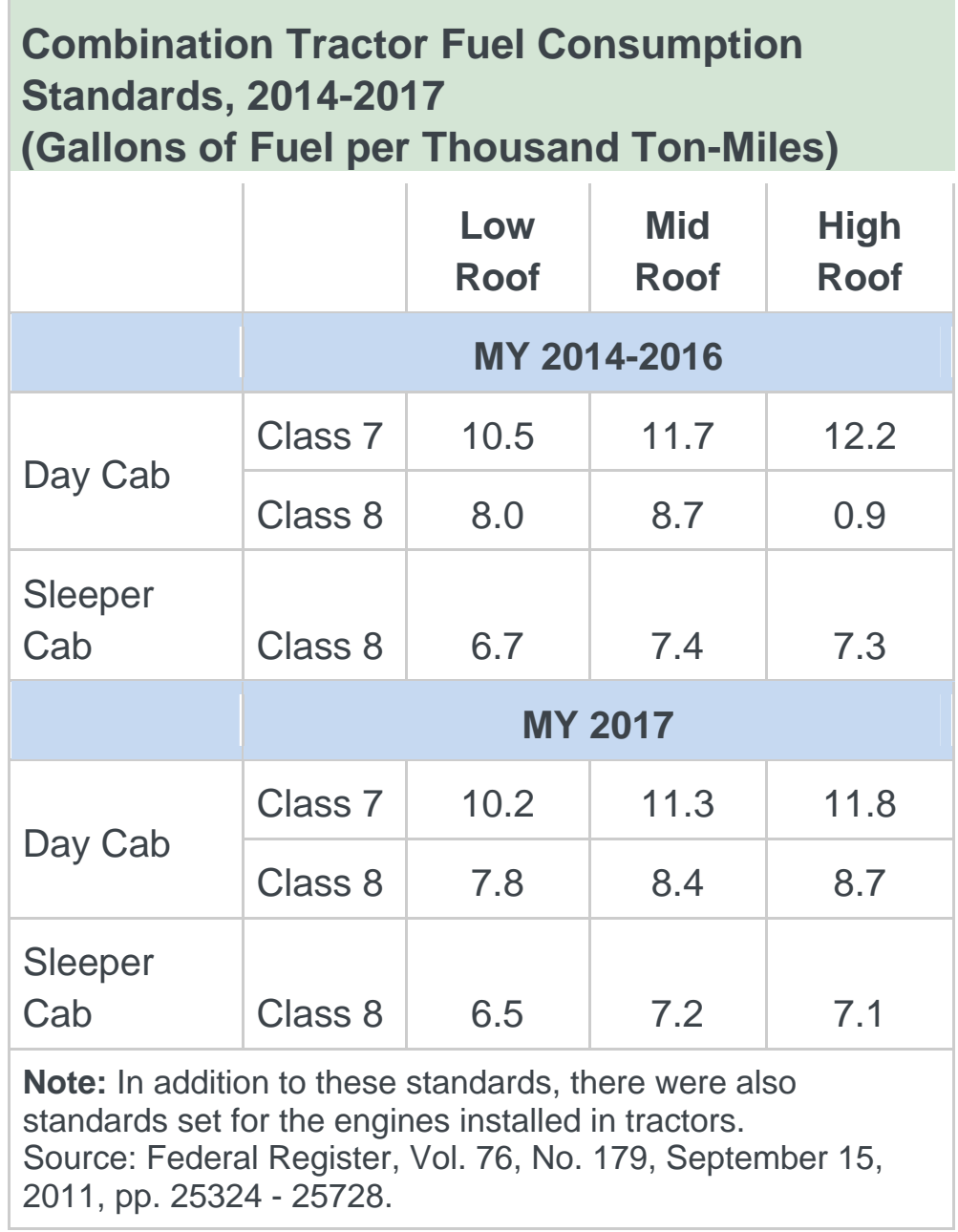




\section{Vehicle Technologies Program}

\section{Fact \#706: December 19, 2011 Vocational Vehicle Fuel Consumption Standards}

The National Highway Traffic Safety Administration recently published final fuel consumption standards for heavy vehicles called "vocational" vehicles. A vocational vehicle is generally a single-unit work vehicle over 8,500 lbs. gross vehicle weight rating (GVWR) or a passenger vehicle over 10,000 lbs. GVWR that is not a combination tractor. These vehicles vary in size, and include smaller and larger van trucks, utility "bucket" trucks, tank trucks, refuse trucks, urban and over-the-road buses, fire trucks, flat-bed trucks, and dump trucks, among others. Often, these trucks are built as a chassis with an installed engine purchased from one manufacturer and an installed transmission purchased from another manufacturer. The chassis is typically then sent to a body manufacturer, which completes the vehicle by installing the appropriate feature-such as dump bed, delivery box, or utility bucket—onto the chassis. Because of the complexities associated with the wide variety of body styles, NHTSA decided to finalize a set of standards beginning in 2016 for the chassis manufacturers of vocational vehicles (but not the body builders).

Vocational Vehicle Fuel Consumption Standards, MY 2016-2017

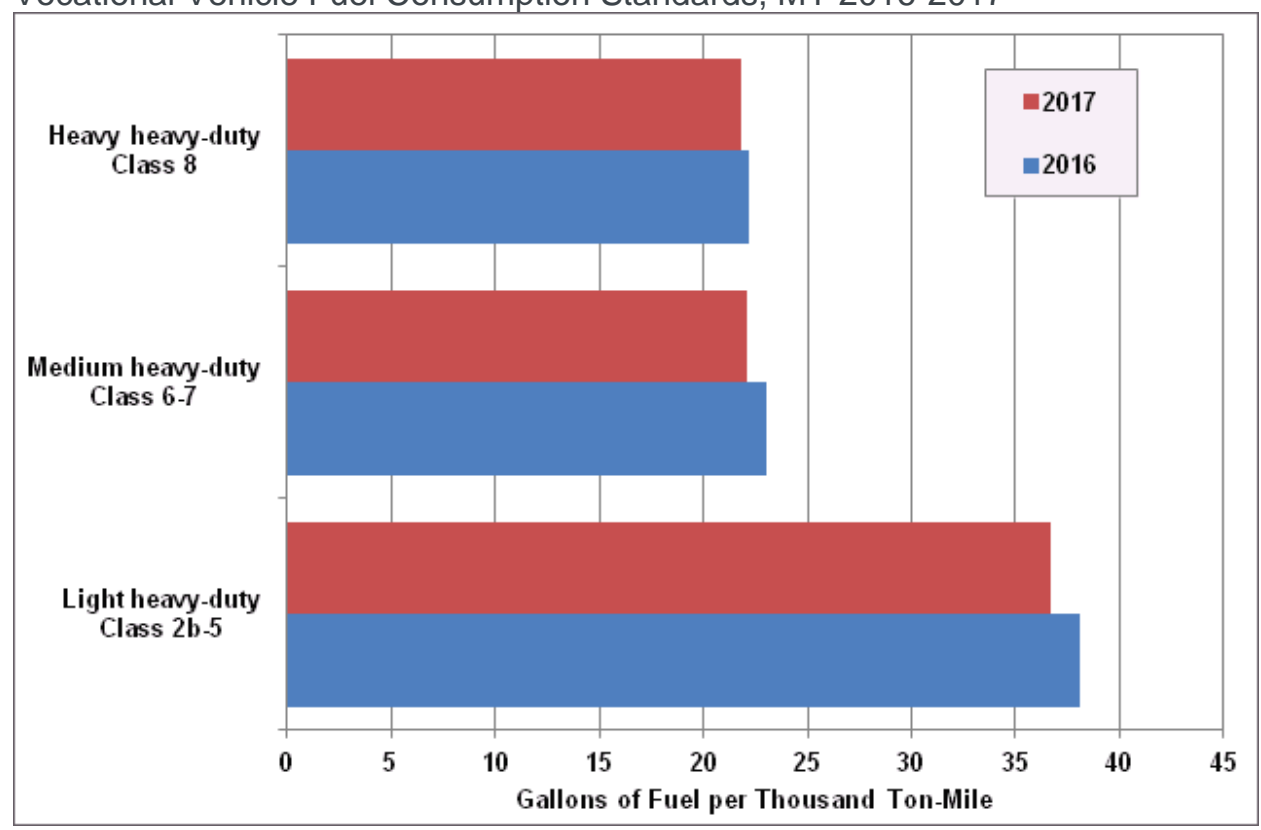

Note: Vehicles in classes $2 b-5$ are between 8,500 and 19,500 lbs. GVWR. Vehicles in class 6-7 are between 19,500 and 33,000 lbs. GVWR. Vehicles in class 8 are above 33,000 lbs. GVWR. 


\section{Supporting Information}

\begin{tabular}{|c|c|c|c|}
\hline Category & Class & 2016 & 2017 \\
\hline $\begin{array}{l}\text { Light heavy- } \\
\text { duty }\end{array}$ & Class $2 b-5$ & 38.1 & 36.7 \\
\hline $\begin{array}{l}\text { Medium } \\
\text { heavy-duty }\end{array}$ & Class 6-7 & 23.0 & 22.1 \\
\hline $\begin{array}{l}\text { Heavy heavy- } \\
\text { duty }\end{array}$ & Class 8 & 22.2 & 21.8 \\
\hline
\end{tabular}

Note: In addition to these standards, there were also standards set for the engines installed in vocational vehicles.

A ton-mile is a measure of shipment weight $x$ distance traveled.

Source: Federal Register, Vol. 76, No. 179, September 15, 2011, pp. 25324 - 25728. 


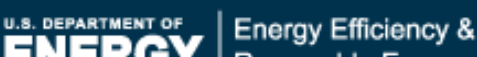 \\ Renewable Energy}

\section{Vehicle Technologies Program}

\section{Fact \#707: December 26, 2011 Illustration of Truck Classes}

There are eight truck classes, categorized by the gross vehicle weight rating (GVWR) that the vehicle is assigned when it is manufactured. These categories are used by the trucking industry and many government agencies to classify trucks. The pictures below show examples of some of the different types of trucks that would be included in each class.

Examples of Trucks in Each Truck Class

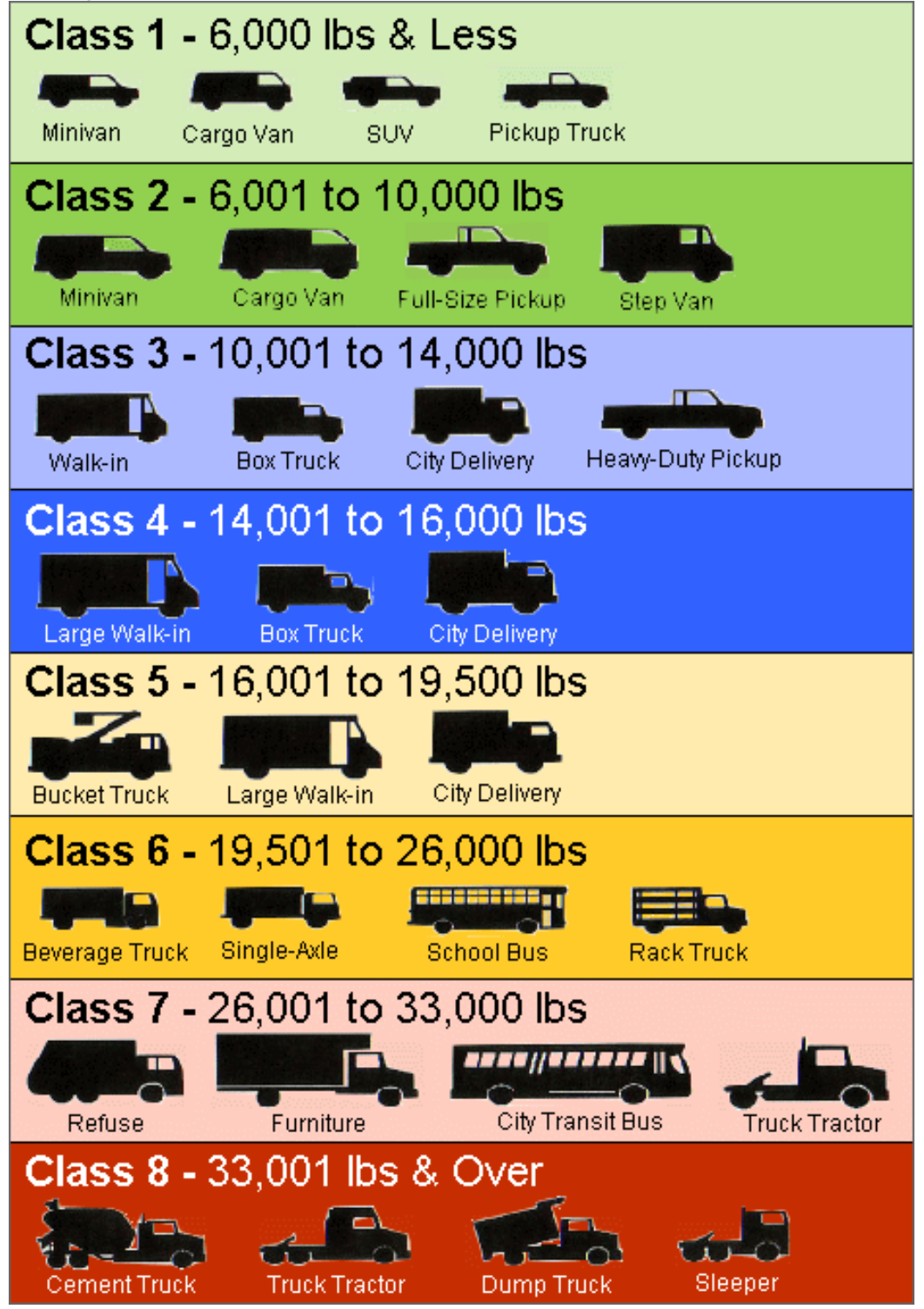




\section{Supporting Information}

\section{Examples of Trucks in Each Truck Class}

Class $1-6,000$ lbs \& Less

\begin{tabular}{|l|l|l|l|}
\hline Minivan & Cargo Van & SUV & Pickup Truck \\
\hline Class 2 -6,001 to 10,000 lbs & \\
\hline Minivan & Cargo Van & $\begin{array}{l}\text { Full-Size } \\
\text { Pickup }\end{array}$ & Step Van \\
\hline Class 3-10,001 to 14,000 lbs & City Delivery & $\begin{array}{l}\text { Heavy-Duty } \\
\text { Pickup }\end{array}$ \\
\hline Walk-in & Box Truck & & \\
\hline Class 4-14,001 to 16,000 lbs &
\end{tabular}

\begin{tabular}{|c|c|c|c|}
\hline Large Walk-in & Box Truck & City Delivery & \\
\hline \multicolumn{4}{|c|}{ Class $5-16,001$ to $19,500 \mathrm{lbs}$} \\
\hline Bucket Truck & $\begin{array}{l}\text { Large Walk- } \\
\text { in }\end{array}$ & City Delivery & \\
\hline \multicolumn{4}{|c|}{ Class $6-19,501$ to $16,000 \mathrm{lbs}$} \\
\hline $\begin{array}{l}\text { Beverage } \\
\text { Truck }\end{array}$ & Single-Axle & School Bus & Rack Truck \\
\hline \multicolumn{4}{|c|}{ Class $7-26,001$ to $33,000 \mathrm{lbs}$} \\
\hline Refuse & Furniture & $\begin{array}{l}\text { City Transit } \\
\text { Bus }\end{array}$ & Truck Tractor \\
\hline \multicolumn{4}{|c|}{ Class 8 - 33,001 lbs \& Over } \\
\hline Cement Truck & $\begin{array}{l}\text { Truck } \\
\text { Tractor }\end{array}$ & Dump Truck & Sleeper \\
\hline
\end{tabular}

Source: Oak Ridge National Laboratory, Center for Transportation Analysis, Oak Ridge, TN. 